OAK RIDGE NATIONAL LABORATORY

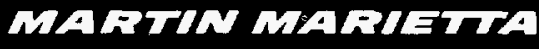
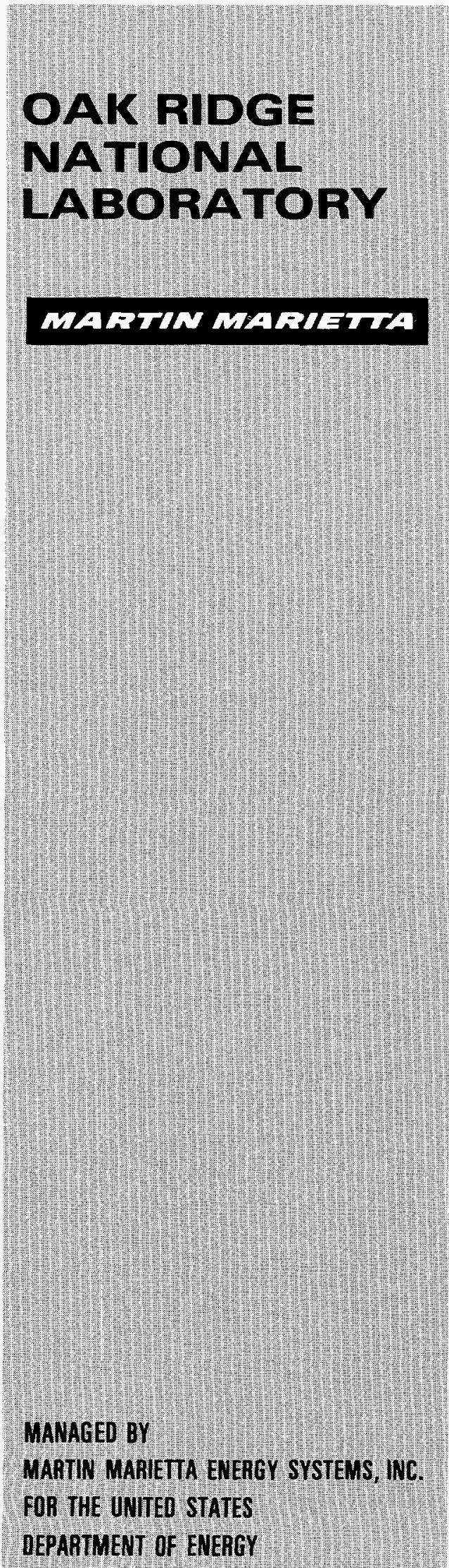

MANAGED BY

MARTIN MARIETTA ENERGY SYSTEMS, INC.

OR THE UNIEU STATES

Q

\section{Traffic Flow Wide-Area Surveillance System Definition}

\author{
Glenn O. Allgood \\ Regina K. Ferrell \\ Stephen W. Kercel \\ Ruth A. Abston \\ Philip I. Moynihan \\ Charles L. Carnal
}

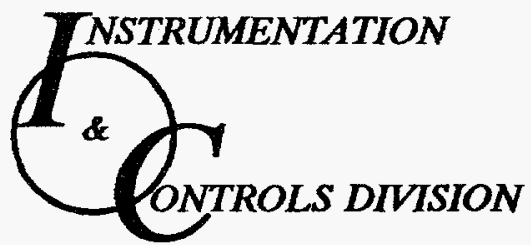


This report has been reproduced directly from the best available copy.

Available to DOE and DOE contractors from the Office of Scientific and Technical Information, P.O. Box 62, Oak Ridge, TN 37831; prices available from (615) 576-8401, FTS 626-8401.

Available to the public from the National Technical Information Service, U.S. Department of Commerce, 5285 Port Royal Rd., Springfield, VA 22161.

This report was prepared as an account of work sponsored by an agency of the United States Government. Neither the United States Government nor any agency thereof, nor any of their employees, makes any warranty, express or implied, or assumes any legal liability or responsibility for the accuracy, completeness, or usefulness of any information, apparatus, product, or process disclosed, or represents that its use would not infringe privately owned rights. Reference herein to any specific commercial product, process, or service by trade name, trademark, manufacturer, or othenwise, does not necessarily constitute or imply its endorsement, recommendation, or favoring by the United States Government or any agency thereof. The views and opinions of authors expressed herein do not necessarily state or reflect those of the United States Government or any agency thereof. 


\section{DISCLAIMER}

Portions of this document may be illegible in electronic image products. Images are produced from the best available original document. 


\title{
Instrumentation and Controls Division
}

\section{TRAFFIC FLOW WIDE-AREA SURVEILLANCE SYSTEM DEFINITION*}

\author{
Glenn O. Allgood \\ Regina K Ferrell \\ Stephen W. Kercel \\ Ruth A Abston \\ Philip I. Moynihant \\ Charles L. Carnal
}

Date Published-November 1994

*This report was part of the Wide-Area Surveillance Systems project sponsored by the U.S. Department of Transportation's Federal Highway Administration.

tJet Propulsion Laboratory, Pasadena, California.

\author{
Prepared for the \\ FEDERAL HIGHWAY ADMINISTRATION \\ Prepared by \\ OAK RIDGE NATIONAL LABORATORY \\ Oak Ridge, Tennessee 37831-6285 \\ managed by \\ MARTIN MARIETTA ENERGY SYSTEMS, INC. \\ for the \\ U.S. DEPARTMENT OF ENERGY \\ under contract DE-AC05-84OR21400
}



LIST OF FIGURES $\ldots \ldots \ldots \ldots \ldots \ldots \ldots \ldots \ldots \ldots \ldots \ldots \ldots \ldots \ldots \ldots \ldots$ vii

LIST OF TABLES $\ldots \ldots \ldots \ldots \ldots \ldots \ldots \ldots \ldots \ldots \ldots \ldots \ldots \ldots \ldots \ldots \ldots \ldots \ldots$

ACKNOWLEDGMENTS $\ldots \ldots \ldots \ldots \ldots \ldots \ldots \ldots \ldots \ldots \ldots \ldots \ldots \ldots \ldots \ldots \ldots$

EXECUTIVE SUMMARY $\ldots \ldots \ldots \ldots \ldots \ldots \ldots \ldots \ldots \ldots \ldots \ldots \ldots \ldots \ldots \ldots$ xiii

1. SYSTEM FUNCTIONS AND ATTRIBUTES $\ldots \ldots \ldots \ldots \ldots \ldots \ldots \ldots, 1$

1.1 INTRODUCTION $\ldots \ldots \ldots \ldots \ldots \ldots \ldots \ldots \ldots \ldots \ldots \ldots \ldots, 1$

1.2 PRIMARY FUNCTION $\ldots \ldots \ldots \ldots \ldots \ldots \ldots \ldots \ldots \ldots \ldots \ldots, 2$

1.3 SAFETY $\ldots \ldots \ldots \ldots \ldots \ldots \ldots \ldots \ldots \ldots \ldots \ldots \ldots \ldots \ldots \ldots \ldots \ldots \ldots \ldots, 2$

1.4 RECONFIGURABILITY $\ldots \ldots \ldots \ldots \ldots \ldots \ldots \ldots \ldots \ldots \ldots \ldots \ldots \ldots \ldots \ldots$

1.5 RELIABILITY $\ldots \ldots \ldots \ldots \ldots \ldots \ldots \ldots \ldots \ldots \ldots \ldots \ldots \ldots \ldots \ldots \ldots \ldots \ldots, 3$

1.6 EXPANDABILITY $\ldots \ldots \ldots \ldots \ldots \ldots \ldots \ldots \ldots \ldots \ldots, 4$

2. TFWAS TECHNOLOGIES $\ldots \ldots \ldots \ldots \ldots \ldots \ldots \ldots \ldots \ldots \ldots, 5$

2.1 MACHINE COGNITION/DATA INTEGRATION ............. 5

2.2 SYSTEMS DESIGN AND LARGE-SCALE INTEGRATION $\ldots \ldots \ldots, 7$

2.3 HUMAN FACTORS $\ldots \ldots \ldots \ldots \ldots \ldots \ldots \ldots \ldots \ldots \ldots, 8$

2.4 CONTROL STRATEGY $\ldots \ldots \ldots \ldots \ldots \ldots \ldots \ldots \ldots \ldots \ldots, 8$

3. COMMUNICATION TECHNOLOGY $\ldots \ldots \ldots \ldots \ldots \ldots \ldots \ldots \ldots, 10$

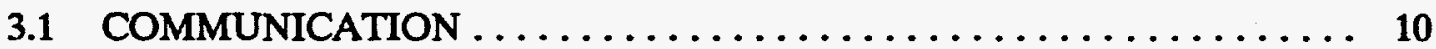

3.2 BASIC COMMUNICATIONS CONCEPTS $\ldots \ldots \ldots \ldots \ldots \ldots \ldots, 10$

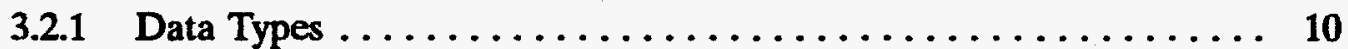

3.2.2 Data Modulation Techniques .................... 10

3.3 DATA TRANSMISSION TECHNIQUES $\ldots \ldots \ldots \ldots \ldots \ldots \ldots \ldots, 12$

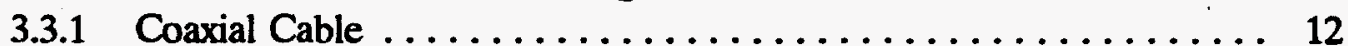

3.3.2 Twisted Pair $\ldots \ldots \ldots \ldots \ldots \ldots \ldots \ldots \ldots \ldots \ldots \ldots \ldots \ldots \ldots \ldots$

3.3.3 Fiber Optics ............................. 16

3.3.4 Meteor Burst Communications $\ldots \ldots \ldots \ldots \ldots \ldots \ldots \ldots \ldots, 18$

3.3.5 Terrestrial Microwave $\ldots \ldots \ldots \ldots \ldots \ldots \ldots \ldots \ldots \ldots \ldots, 20$

3.3.6 Satellite $\ldots \ldots \ldots \ldots \ldots \ldots \ldots \ldots \ldots \ldots \ldots \ldots \ldots, 21$

3.3.7 Cellular Radio .................................... 23

3.3.8 Packet Radio $\ldots \ldots \ldots \ldots \ldots \ldots \ldots \ldots \ldots \ldots \ldots \ldots \ldots, 25$

3.3.9 Spread-Spectrum Radio ......................... 26

3.3 .10 Other $\ldots \ldots \ldots \ldots \ldots \ldots \ldots \ldots \ldots \ldots \ldots \ldots \ldots \ldots \ldots \ldots \ldots \ldots \ldots, 27$

3.3.11 Conclusions $\ldots \ldots \ldots \ldots \ldots \ldots \ldots \ldots \ldots \ldots \ldots \ldots, 28$

4. SENSOR TECHNOLOGIES OVERVIEW .................. 31

4.1 SENSOR CHARACTERISTICS AND THEIR IMPLICATIONS ...... 31

4.1.1 Sensor Placement-Pavement-Based Versus

Aboveground Technologies .................... 32

4.2 TRAFFIC PARAMETER DEFINITIONS $\ldots \ldots \ldots \ldots \ldots \ldots \ldots \ldots, 34$ 


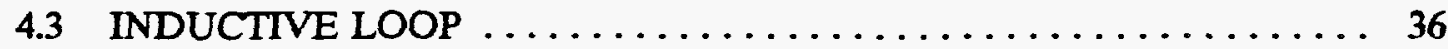

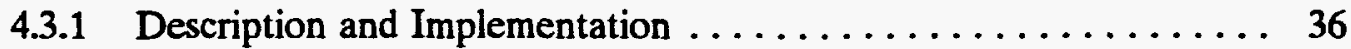

4.3.2 Advantages and Disadvantages $\ldots \ldots \ldots \ldots \ldots \ldots \ldots \ldots \ldots, 36$

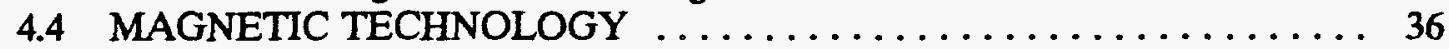

4.4.1 Description and Implementation .................. 36

4.4.2 Advantages and Disadvantages $\ldots \ldots \ldots \ldots \ldots \ldots \ldots \ldots \ldots \ldots \ldots \ldots$

4.4.3 Present Research Efforts ...................... 37

4.5 MICROWAVE TECHNOLOGY $\ldots \ldots \ldots \ldots \ldots \ldots \ldots \ldots \ldots \ldots \ldots \ldots \ldots \ldots$

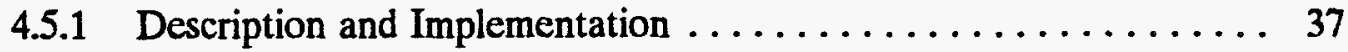

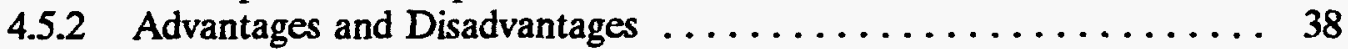

4.5.3 Present Research Efforts ....................... 39

4.6 ULTRASONIC TECHNOLOGY $\ldots \ldots \ldots \ldots \ldots \ldots \ldots \ldots \ldots \ldots, 40$

4.6.1 Description and Implementation $\ldots \ldots \ldots \ldots \ldots \ldots \ldots \ldots \ldots, 40$

4.6.2 Advantages and Disadvantages $\ldots \ldots \ldots \ldots \ldots \ldots \ldots \ldots \ldots, 40$

4.6.3 Present Research Efforts . . . . . . . . . . . . . . . 40

4.7 PASSIVE ACOUSTICS ..................... 40

4.7.1 Description and Implementation $\ldots \ldots \ldots \ldots \ldots \ldots \ldots \ldots \ldots, 40$

4.7.2 Advantages and Disadvantages .................. 41

4.7.3 Present Research Efforts $\ldots \ldots \ldots \ldots \ldots \ldots \ldots \ldots \ldots \ldots .41$

4.8 INFRARED TECHNOLOGY $\ldots \ldots \ldots \ldots \ldots \ldots \ldots \ldots \ldots \ldots, 41$

4.8.1 Description and Implementation $\ldots \ldots \ldots \ldots \ldots \ldots \ldots \ldots \ldots \ldots \ldots \ldots \ldots \ldots \ldots$

4.8.2 Advantages and Disadvantages $\ldots \ldots \ldots \ldots \ldots \ldots \ldots \ldots \ldots, 42$

4.8.3 Present Research Efforts ...................... 42

4.9 VIDEO IMAGE PROCESSING $\ldots \ldots \ldots \ldots \ldots \ldots \ldots \ldots \ldots \ldots, 42$

4.9.1 Description and Implementation $\ldots \ldots \ldots \ldots \ldots \ldots \ldots \ldots, 42$

4.9.2 Advantages and Disadvantages $\ldots \ldots \ldots \ldots \ldots \ldots \ldots \ldots \ldots, 43$

4.9.3 Present Research Efforts ..................... 44

4.10 OTHER SENSING TECHNIQUES AND SPECIAL SUBSETS $\ldots \ldots \ldots 45$

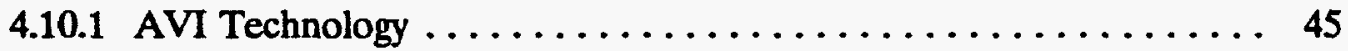

4.10 .2 Probe Vehicles $\ldots \ldots \ldots \ldots \ldots \ldots \ldots \ldots \ldots \ldots \ldots \ldots, 46$

4.10 .3 Synthetic-Aperture Radar . . . . . . . . . . . . . . . 47

4.10.4 3-D Laser Imaging $\ldots \ldots \ldots \ldots \ldots \ldots \ldots \ldots \ldots \ldots \ldots \ldots \ldots \ldots \ldots \ldots$

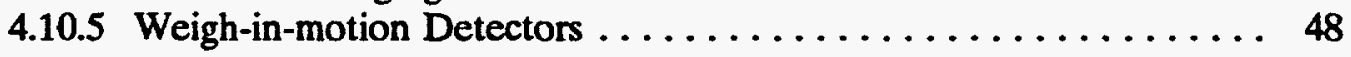

5. EVALUATION OF TECHNOLOGY CAPABILITIES

FOR TFWAS SYSTEM REQUIREMENTS $\ldots \ldots \ldots \ldots \ldots \ldots \ldots \ldots \ldots, 49$

5.1 OVERVIEW OF TECHNOLOGIES $\ldots \ldots \ldots \ldots \ldots \ldots \ldots \ldots \ldots, 49$

5.2 TECHNOLOGY CAPABILITIES FOR TFWAS $\ldots \ldots \ldots \ldots \ldots \ldots .59$

5.3 SENSOR MEASUREMENT OF AGGREGATE PARAMETERS . . . . 61

6. MEASURES OF PERFORMANCE $\ldots \ldots \ldots \ldots \ldots \ldots \ldots \ldots \ldots \ldots \ldots \ldots$

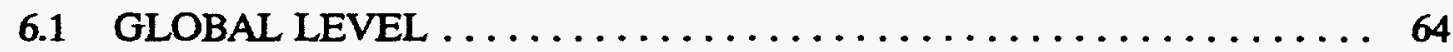

6.2 SYSTEM LEVEL $\ldots \ldots \ldots \ldots \ldots \ldots \ldots \ldots \ldots \ldots \ldots \ldots \ldots \ldots, 65$

6.3 SUBSYSTEM LEVEL $\ldots \ldots \ldots \ldots \ldots \ldots \ldots \ldots \ldots \ldots \ldots \ldots \ldots, 66 \ldots$

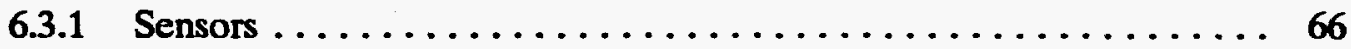

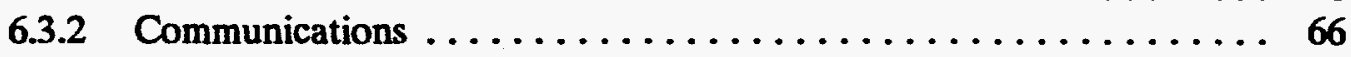

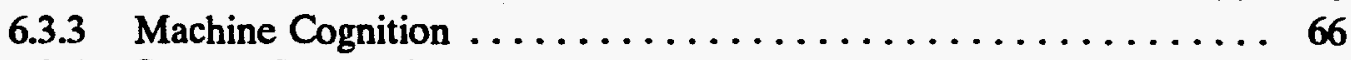

6.3 .4 Systems Integration ....................... 67 
7. CONCLUSIONS $\ldots \ldots \ldots \ldots \ldots \ldots \ldots \ldots \ldots \ldots \ldots \ldots \ldots \ldots$

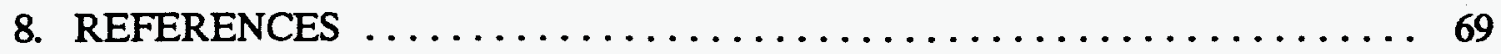

Appendix A: CAPABILITIES OF NATIONAL LABORATORIES RELEVANT

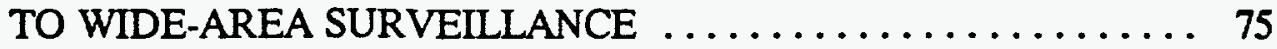

Appendix B: A LOOK TO THE FUTURE $\ldots \ldots \ldots \ldots \ldots \ldots \ldots \ldots \ldots$

Appendix C: ILLUSTRATION OF DIFFERENCES BETWEEN REACTIVE

AND ANTICIPATORY SYSTEMS . . . . . . . . . . . 91 



\section{LIST OF FIGURES}

Fig. 2.1. Major technologies involved in creating a TFWAS $\ldots \ldots \ldots \ldots \ldots$

Fig. 4.1. Levels of information abstraction $\ldots \ldots \ldots \ldots \ldots \ldots \ldots \ldots$ 



\section{LIST OF TABLES}

Table 3.1 Comparison of the data transmission characteristics for the various

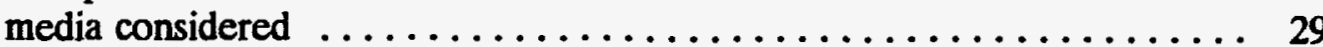

Table 3.2 Cost comparison of the owned communications media considered $\ldots \ldots 30$

Table 3.3 Cost comparison of leased communications media $\ldots \ldots \ldots \ldots \ldots \ldots$

Table 5.1 System considerations for TFWAS system technology $\ldots \ldots \ldots \ldots \ldots \ldots$

Table 5.2 Estimated cost considerations for TFWAS system technology $\ldots \ldots \ldots .53$

Table 5.3 Statistical and systems measures for TFWAS system technology $\ldots \ldots \ldots 54$

Table 5.4 Degree to which aggregate traffic parameters can be determined by TFWAS technology $\ldots \ldots \ldots \ldots \ldots \ldots \ldots \ldots$ 



\section{ACKNOWLEDGMENTS}

The project team is grateful to the U.S. Department of Transportation's Federal Highway Administration for their sponsorship of this preliminary wide-area surveillance study. 



\section{EXECUTIVE SUMMARY}

Traffic Flow Wide-Area Surveillance (TFWAS) is a system for assessing the state of traffic flow over a wide area for enhanced traffic control and improved traffic management and planning. The primary purpose of a TFWAS system is to provide a detailed traffic flow description and context description to sophisticated traffic management and control systems being developed or envisioned for the future. The TFWAS system will act as a transducer for the traffic control system and should be developed with the flexibility to evolve and integrate with the Intelligent Vehicle Highway System.

A successful TFWAS system must possess the attributes of safety, reconfigurability, reliability, and expandibility. The primary safety premise of TFWAS is to ensure that no action or failure of the TFWAS system or its components can result in risk of injury to humans. Hardware reconfigurability implies a modularity of the sensor suite-sensors are easy to plug into and unplug from the overall system. A second type of reconfigurability involves the hierarchy of control-sometimes multiple systems sensor suites are integrated to operate as one large TFWAS system; and other times, they operate autonomously as separate TFWAS systems. System reliability can be ensured by various techniques such as fault-tolerant computing and incipient failure detection. (Incipient failure detection would allow change outs or bypasses of components before a failure occurs.) Upward compatibility with dynamically reconfigurable control must be included in the system from the original design to ensure expandability.

TFWAS is a conceptual step beyond high-tech sensor development or signal and data processing performed on a collection of sensors. It is an integrated, robust sensory system that depends critically on seven key technologies. The key technologies involved with successful implementation of TFWAS include communications, sensors, machine cognition/data integration, control strategies, systems design, large-scale system integration, and human factors.

A wide variety of communication techniques is available for use with TFWAS systems. These communication techniques can be broken down into two categories, landlines and wireless. Landlines include the low-bandwidth twisted pair, the higher bandwidth coaxial cable, and fiber optics. Data can also be communicated in a wireless fashion electromagnetically. These waves can be transmitted from point to point by satellite, meteor burst, or a series of terrestrial microwave transponders. Radio waves can also be transmitted to and from mobile transponders by using satellites or terrestrial antennas. Narrowband or spread-spectrum technology can be used. Commercially provided services such as cellular or packet radio can be used to take advantage of the existing communication infrastructures. These services can be compared on the basis of costs and data transmission characteristics such as bandwidth, data rate, and repeater distance.

With the above concepts in mind, currently used and possible future traffic sensing technologies have been examined. Important criteria for selecting TFWAS sensors include sensor capabilities, costs, operational constraints, sensor compatability with the infrastructure, and extent. The primary sensor technologies discussed include inductive loops, magnetic devices, microwave devices, ultrasonic devices, acoustic devices, infrared devices, optical image processing devices, and weigh-in-motion devices. Special sensing categories or subtechnologies discussed include three-dimensional laser imaging systems, synthetic aperture radar, automatic vehicle identification systems, and probe vehicles. The capabilites of each of these sensors for providing TFWAS measures have been analyzed and discussed. 
Several measures of performance will be applied to assess the performance of a TFWAS system, both on a global level and at a systems level. On a global level, the overall effectiveness of a TFWAS system will be assessed by how the operation of such a system changes measurable traffic flow parameters. At a systems level, the performance of the TFWAS system will be assessed relative to how well the TFWAS system measures and communicates data. The individual components of a TFWAS system will be assessed as well, with various parameters used to measure the performance of the sensor suites, the communications, and the processing algorithms.

Because the TFWAS system can be thought of as a transducer for the traffic flow control system, many of the same overall measures of performance used to assess the traffic flow control system can be used to assess a TFWAS system. An objective of a traffic flow control system is to simultaneously minimize delay and maximize throughput, while equalizing the traffic load. A secondary objective is to reduce energy consumption and improve air quality. It is likely that parameters such as link travel time, vehicle miles traveled, and "slug" velocity and acceleration will be measured and used to assess the performance of a TFWAS system.

At the systems level, the performance of a specific surveillance system is a measure of how well the system provides data. The measures of a system's performance include reliability, reproducibility, accuracy, and repeatability. Repeatability refers to the system's ability to provide the same output for repeated instances of a given input. Reproducibility is associated with repeatability. Reproducibility is defined as the ability of different units to provide the same output for a given input. Accuracy is a measure of the deviation of the result from the true value. Reliability is a measure of the availability of the system. Reliability includes such things as mean time between failures and percent downtime.

TFWAS is a concept that can take advantage of the strengths of different traffic sensing technologies, can readily adapt to newly developed technologies, and can grow with the development of new traffic control strategies. By developing innovative algorithms that will take information from a variety of sensor types and develop descriptions of traffic flows over a wide area, a more comprehensive understanding of the traffic state can be provided to the control system to perform the most reasonable control actions over the entire wide area. The capability of characterizing the state of traffic over an entire region should revolutionize developments in traffic control strategies. 


\section{SYSTEM FUNCTIONS AND ATTRIBUTES}

\subsection{INTRODUCTION}

A universal Traffic Flow Wide-Area Surveillance (TFWAS) system suitable for all applications and environments is not a practical, cost-effective solution for traffic sensing. Besides the varying requirements based on climate, roadway type, and physical surroundings, the enabling technologies are changing too rapidly for any particular configuration to be pronounced as the definitive TFWAS system for all applications. This document sets forth the principles by which a TFWAS system could be defined, given knowledge of the context and the state of the available technologies.

The objective of the system definition phase of this effort is to provide a long-term view of the issues affecting the evolution of TFWAS technology. Although this document includes a fair amount of detail about the relative merits of applicable present-day technologies (especially sensors), an assessment of the present state of the TFWAS system art is merely the starting point in describing its future direction. This document is primarily concerned with why TFWAS systems are needed, what constrains their deployment and operation, and what technologies must be applied to deploy such a system.

The National Program Plan for Intelligent Vehicle Highway System (NPP 1994) is an effort to devise a cohesive plan for the entire Intelligent Vehicle Highway System (IVHS), incorporating all relevant issues. Although this plan is still in draft form, it probably represents the best available statement of the expected wants and needs of the IVHS community over the next several decades. The NPP (1994) starts to answer the question of why TFWAS systems are needed.

According to the NPP (1994), three services require the information provided from a TFWAS system. Primarily, the system is required to provide traffic flow information to the sophisticated adaptive traffic control systems that are under development. A tangential, urgently needed by-product of traffic flow information is automatic incident detection. Somewhat further in the future, traffic flow information can be used to update real-time route guidance schemes.

Systems being represented as "Traffic Flow Wide-Area Surveillance" are already being sold commercially and deployed. These systems consist primarily of sensor arrays with some signal processing. They do not provide the cognitive description of traffic flow that will be required by future generations of traffic control systems. The biggest constraints on the development of an intelligent TFWAS system are lack of knowledge of the IVHS architecture and lack of cognitive algorithms to deduce the maximum available traffic flow information from the sensor data.

Two major tasks need to be completed to deploy a TFWAS system. One is completion of the System Architecture Development Study, which the Federal Highway Administration (FHWA) should finish within the next 2 years. The other task is the development of anticipatory and cognitive algorithms that will provide the intelligence in a TFWAS system. Although this task would be a very difficult effort, it would have an immense payoff. Even with aggressive support and effort, this task might take a decade. 


\section{PRIMARY FUNCTION}

The primary function of a TFWAS system is to provide a description of traffic conditions to a traffic control system. This description must include both (1) traffic data, such as velocities, densities, and queue lengths, and (2) context data, such as time of day and weather conditions. In a full-featured TFWAS system, the specific attributes or structure of the description passed to the control system can be dynamically altered upon demand by the control system.

The TFWAS system and the control system will also interact with a human-operated traffic management center (TMC). Thus, in addition to generating a machine-readable conceptualization of the flow pattern, the TFWAS system must also be capable of generating a quickly comprehendable verbal/graphical interpretation of the flow pattern.

Initial TFWAS system requirements presume that vehicles are totally passive participants in the traffic sensing process. Thus, initially, the TFWAS system must perform its task without any participation from individual vehicles. It is anticipated that vehicles will gradually acquire intelligent sensing and response capability. At some stage of TFWAS system development, the TFWAS system feature descriptions could feed a route guidance system that reduces congestion and provides input to the TFWAS system as to the volume and entry points of traffic about to enter the system. Note that even when available, this service is expected to have the desired effect of reducing overall congestion and creating a more favorable context in which to operate the traffic control system. However, because the TFWAS system feeds information into a route guidance system and eventually the traveler acts on it, the control action itself has an extremely slow time constant. This slow response is compounded by the fact that the driver may or may not act as recommended by the control system. In a later evolution of the traffic control system, the "smart" highway may directly affect control of the "smart" vehicle, such as by organizing platoons in real time. In this case, control actuations mean that the traffic control system has physical control of individual vehicles. The traffic control time constant could be much shorter with such a system. This implementation is decades in the future, but the initial implementations of TFWAS systems and traffic control systems need to be upwardly compatible with these anticipated features.

\section{SAFETY}

The safety objective of the TFWAS system is "first, do no harm." That is, the TFWAS system must not add risk from either a performance or a human user's standpoint. Within the purview of "first, do no harm," fail-safe considerations must be included, such as designing a traffic control signal so that it does not display green in all directions when it fails. It is intended and expected that by improving the continuity of traffic flow, traffic control will become safer.

Safety of the overall system must be examined with respect to its relationship to traffic management and control systems. For example, what are the consequences (1) of an inaccurate description of the state of traffic or (2) of information gleaned from a malfunctioning sensor? What type of control decisions and actions are made as a result of information from the TFWAS system? Can these actions cause accidents or harm to system users? The consequences of inaccurate surveillance information may change dramatically as the control system evolves. The accuracy and redundancy of the surveillance system becomes particularly important if the control system evolves to directly controlling vehicles, managing 
platoons, etc. At each stage of evolution of the system, a safety analysis must be performed to ensure a continuing level of safety.

\subsection{RECONFIGURABILTY}

The NPP (1994) anticipates that the traffic control system will have a dynamically reconfigurable architecture. Therefore, any system supporting traffic control and management should be hierarchical and modular in design. An essential attribute of TFWAS system design is reconfigurability. Three kinds of reconfigurability can be identified. The first is reconfigurability of the sensor suite; sensors are easy to plug and unplug. The second is reconfigurability of the hierarchy of control; sometimes multiple systems operate as one, and other times they operate autonomously. Finally, a system might reconfigure both its control architecture and information flows in real time in response to a changing context.

A dynamically reconfigurable sensor interface adds modularity to the design effort. Hardware should be provided to interface a variety of sensors to a general input/output (I/O) data bus in the TFWAS system. Software drivers should also be provided for each interface, along with an intelligent interface module that can determine what sensors are on the system. The design should allow convenient addition or deletion of sensors from the system while on line.

Reconfigurability of hierarchy of control implies that the boundaries of a wide area may expand or shrink under various traffic conditions to optimize an overall control strategy. Data outputs from various sensor suites and information flows between processors must be able to be integrated to optimize traffic descriptions.

Reconfiguration of information flows in response to a changing context is a capability that adds a great deal of flexibility to the TFWAS system. On the basis of traffic flow information it receives, the control system determines whether a different set of measures or information is more useful and requests this change to the TFWAS system. The TFWAS then restructures its traffic description to better meet the needs of the control system.

\section{RELIABILITY}

The reliability of the system and its components is an issue. One measure of TFWAS system performance will be what percentage of time the cognitive machinery and the individual sensors are available and functioning. The system should include incipient failure detection. It should monitor its subsystems and detect the trends which indicate that although the subsystem is still operating within acceptable limits, it will fail soon. This monitoring will improve the reliability of the system by allowing change outs or bypasses before the failure occurs. It will also reduce maintenance costs by indicating what parts of the system actually need maintenance.

The modularity and reconfigurability of the system will allow rerouting around subsystems that have either failed or are about to fail. This reconfigurability, combined with the robustness of the cognitive machinery (cognition does not require perfect or complete sensor data), should preclude the need for a major backup system. Unless everything fails at once, the system should not experience catastrophic failure. 


\subsection{EXPANDABILITY}

System implementation will begin by deploying small TFWAS systems that are expected to grow. I/O interfaces that are easy to expand and processing algorithms that are scalable will be required. The property of scalability means that the computational cost of an algorithm is roughly proportional to the amount of information it processes. Although suitable to monitor one intersection, an algorithm whose execution time grows factorially as a function of area may fail miserably when expanded to monitor four intersections at once.

Growth has another implication: the interconnectivity required to integrate small, local TFWAS systems into a cohesive system. Upward compatibility with dynamically reconfigurable control must be included in the system from the beginning. That is, when several TFWAS systems grow until they begin to merge with others, it should be an easy transition to integrate them into a single system when the context demands it, to run them autonomously when the context demands it, and to switch back and forth as conditions change. 


\section{TFWAS TECHNOLOGIES}

TFWAS systems may vary from site to site based on the physical attributes of the wide area, on the extremeties of weather conditions under which the system must operate, and on the traffic knowledge requirements of the control system for the wide area. To meet these requirements, a whole series of TFWAS systems could be developed for several different kinds of defined physical settings. A better way to meet these criteria would be to develop a hierarchical modular system that can be customized to a physical setting by judicious selection of modules. The structure of the TFWAS system and its essential attributes should meet a basic standard. The basic components of a TFWAS system as shown in Fig. 2.1 include a suite of sensors, a data integration or cognition module, an interface to the control system, and communications modules both internal and external to the TFWAS system. Human factors and systems integration are technologies that must be integrated throughout the TFWAS system design.

Communications and sensing technologies are examined in detail in later chapters of this document. The other components of the system are covered in the remainder of this chapter.

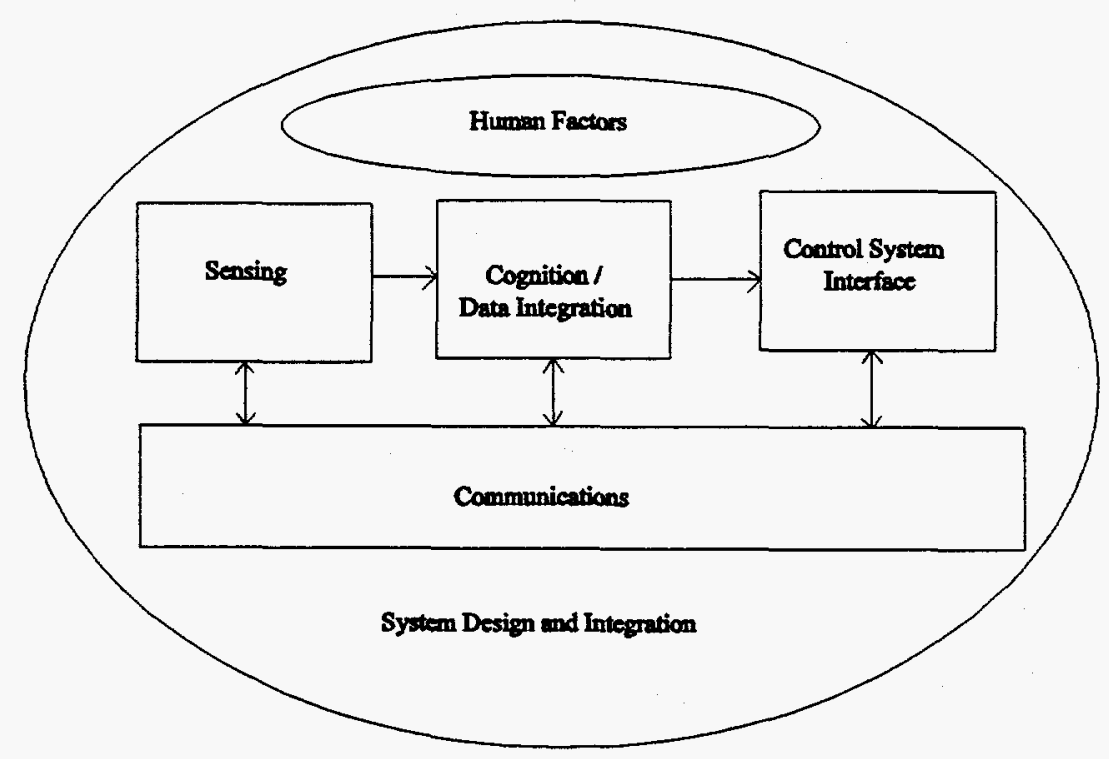

Fig. 21. Major technologies involved in creating a TFWAS.

\subsection{MACHINE COGNITION/DATA INTEGRATION}

The application of machine cognition to the problem of TFWAS must go beyond shortterm applications. The NPP (1994) recognizes that in the full-featured TFWAS, required as the transducer into a full-featured adaptive traffic control system in a fully implemented IVHS, the major unsolved problem is machine cognition. How does the surveillance system 
take a large set of data flows and from those determine the pattern of traffic flow through the area under surveillance?

In the short term, a solution can be offered to a subset of the problem. Data fusion algorithms exist that make no pretense at being cognitive algorithms (Klein 1993). Although not cognition, these fusion algorithms are, nevertheless, useful. Through the judicious use of fusion algorithms running on progressively faster and cheaper computers, dramatic improvements can no doubt be made in the ability of the present generation of "wide-area surveillance" systems to extract the maximum amount of information from the data being furnished by their sensor arrays. Presently available data fusion algorithms represent a means of improving the percepts delivered by the present generation of traffic flow sensors; they do not present a conceptual view of traffic flow.

Earlier implementations of TFWAS will provide more limited performance with presently available algorithms. Specific algorithms have been developed to perform limited functions in precisely defined contexts. In a general-purpose cognition algorithm, these kinds of functions would become special cases. The full-featured TFWAS one or two generations in the future will need to provide a conceptual view of the traffic flow pattern. Nobody knows how to write a machine cognition algorithm yet. Indeed, some philosophers argue that it cannot be done. If it is to happen, the research must start now.

There are many "proofs" that machine cognition is impossible to implement (Copeland 1993). Despite philosophical and scientific "proofs" that a technological advance is impossible to implement, it is good to keep in mind how often these arguments have been proven false in the past. At one time or another, it was part of the scientifically proven conventional wisdom that human ground travel at more than $20 \mathrm{mph}$, supersonic air travel, and human space travel were all impossible. What these "proofs" offered was merely that if the problem is constrained by the premises assumed at the beginning of the proof, then the problem cannot be solved when bound by those constraints. In other words, a thinking machine will be considered impossible by the conventional wisdom until somebody builds one.

Artificial intelligence researchers who are undeterred by assurance that their cause is hopeless have been recently investigating machine intelligence along four different paths (Carbonell 1992). The one that seems the most promising for TFWAS is the inductive paradigm, in which the machine learns a concept from repeated exposure to instances of, and counterexamples to, the concept. This is somewhat akin to the idea of an unsupervised pattern recognition algorithm, in which, given a sufficient number of instances of different classes of patterns, the algorithm recognizes the natural clusters that they form. The TFWAS system is continually exposed to instances of traffic flow, is expected to draw conclusions from what is sees, but is provided with very little feedback as to whether or not it got the right answer.

The analytic paradigm does not seem promising for TFWAS. In the analytic paradigm, the system is given very few examples and a long list of theoretical principles. This seems to be the opposite of TFWAS, which has a torrent of data and relatively few principles.

The genetic paradigm probably lacks the resolution to provide the information needed to characterize traffic flow. It is a pattern recognition scheme, assigning instances of input data into distinct classes. It might not be able to deal with enough different classes to handle all the possible different descriptions of traffic flow.

The connectionist paradigm is an elegant name for the ever-popular neural network. It suffers the same limitation as the genetic paradigm; it is a classifier and may not support enough different classes to be usable for TFWAS. It also suffers from the problem that many 
people think it can be used as a substitute for understanding of the underlying physical process. It cannot.

A number of recent developments of special cases could begin to lay foundations for cognition algorithms for highway surveillance. These include incident detection using fuzzy set theory (Chang and Wang 1994), incident detection using Kalman predictor (Dailey 1994), traffic volume and classification monitoring (Mead et al. 1994), link travel times from point detector readings (Sisiopiku and Rouphail 1994), start-stop waves using cellular automata (Nagel and Schreckenberg 1994), and formalized data fusion algorithms (Klein 1993).

\section{SYSTEMS DESIGN AND LARGESCALE INTEGRATION}

Although listed as two distinct technological areas, in practice, it is difficult to separate systems design and large-scale integration. Although a full-featured TFWAS system would represent a new engineering application of these areas, it is not expected that any major technological breakthroughs are needed. Extensive research in large, integrated, paralleldistributed systems has been conducted in the area of ballistic missile defense. Mariani (1984) presents a concise summary of the issues involved.

Because of its physical extent, a TFWAS system is necessarily distributed. Because many individual sensors have local processing, it is in some sense a parallel processor. One can envision two different extremes in parallel-distributed processing. At one extreme is a system like a local area network of personal computers. The processing is locally done by processors operating nearly autonomously, with occasional communications with other processors. Performance in this kind of system is typically limited by the processor performance. At the other extreme of distributed-parallel processing is a localized parallel processor, such as the Intel Hypercube. Here, the process itself is distributed, often using algorithms specifically designed for parallel architectures. Such a system uses frequent communications between processes and processors, and interprocess communications impose the limit on performance on the system.

A TFWAS system tends to fall between these two extremes. The interprocess communications load falls somewhere between a network of autonomous computers and a dedicated parallel processor. Consequently, it is not clear whether the processors or the interprocess communications are the limiting factor on performance.

Mariani (1984) identifies the following critical issues that must be addressed in the implementation of a distributed system. These are not the only issues; however, they must be successfully addressed to ensure productive development of a TFWAS system.

- Real-Time Control: For each local processor, and for each process level, how will the process and the resource allocation be controlled?

- Decomposition/Partitioning: How will the overall task be distributed between processes?

- Distributed Database Design: How should the database be designed?

- Intranode Communication: In particular, what protocols lead to the highest throughput?

- Fault Tolerance: What design and control techniques should be used to route around local failures?

- Architecture: What architecture is best suited for this specific application while allowing both high performance and high growth?

- Performance Modeling: How is the system modeled to provide the data to indicate how well the implemented system is really performing? 


\section{HUMAN FACTORS}

Although a full-featured TFWAS system would represent a new engineering application of human factors, it is not expected that any significant technological breakthroughs are needed. The primary problem to be solved is to present the traffic flow pattern to the human operator at a TMC in such a way that it makes sense. Extensive research in the problem of presenting vast amounts of electronic sensor data to a human operator has been conducted in the area of power plant control. Rasmussen (1986) presents a concise summary of the issues involved.

In addition, a major study of human factors issues in automated traffic control is currently being conducted by FHWA for the Automated Highway System (AHS) project. The TurnerFairbank Center is sponsoring a project by a team led by Honeywell which includes the Berkeley/PATH Program, the University of Iowa, and Interscience America. The Contracting Office Technical Representative is Beth Alicandri at Turner-Fairbank. The Principal Investigator is Tom Plocher of Honeywell. Work should be completed by January 1996.

The objective of the project is to develop a human factors design handbook (Plocher 1993). The handbook has three purposes: (1) to support highway and automotive engineers in AHS design, (2) to aid human factors professionals, and (3) to ensure that AHS designs accommodate the traveling public. It is appropriate to note that the emphasis of this study is on the interaction between the driver and the intelligent vehicle.

\section{CONTROL STRATEGY}

Control strategies for wide-area traffic control must incorporate two stochastic aspects of the traffic system. First, the underlying system to be controlled is predominantly modeled by a large, complex network of queues (DOT 1994). Secondly, each item in the set of sensor data has an associated confidence that is usually expressed as the reciprocal of the variance or uncertainty (Shanmugan and Breipohl 1988). Both issues will profoundly influence the design of the architecture of a TFWAS system. The locations and types of sensors will affect the observability and controllability of the control strategy. It will be incumbent on a TFWAS system to provide estimates of the measurement uncertainties for use in control laws. These issues will have a direct bearing on the quality, reliability, and safety of the operation of a traffic control system.

In general, traffic systems are not described by deterministic differential or difference equations and therefore do not lend themselves to control with conventional state space control laws. In recent years, a variety of techniques has emerged to deal with dynamical systems that are driven by discrete events such as networks of queues (Cassandras 1993). In simple cases where queues are widely dispersed or traffic is light, arrival times and service times in the queues are exponential random variables (or, equivalently, the event process is Poisson). Such queues are known as Markovian queues and can be modeled with Markov chains. Dynamic programming (Bertsekas 1987) is an approach that provides optimal control laws for systems described by Markov chains. In more congested traffic scenarios, Non-Markovian queuing theory is required. In such cases, simulation is a starting point for developing optimal control laws. The Monte Carlo method (Rubinstein 1986) is a computationally expensive, if not intractable, approach to obtaining an optimal control strategy. A more direct approach uses perturbation analysis (Glasserman 1991; Ho and Cao 1991) to develop the control laws. 
Whatever control strategy is pursued, massive computational resources are going to be required. That does not necessarily mean that massive computers are required. The computational burden may be distributed with-and be an integral part of-the array of sensors that comprise the TFWAS system. One possible future scenario would find some form of artificial intelligence, or machine cognition as an integral part of each sensor (Haykin 1994; Hecht-Nielsen 1991; Kosko 1992). Hence, the design of a TFWAS system should proceed interactively with the design of the large-scale traffic control system. As a minimum objective, for the sake of control, the architecture of the surveillance system should allow an orderly evolution to a distributed control system.

The treatment of feedback measurements in control laws can be placed into two categories (Astrom and Wittenmark 1989). The simplest approach relies on the certainty equivalence principle; namely, uncertainties of feedback measurements are not taken into account in the control laws. Methods that utilize the statistical confidence in the measurements are generally classified as stochastic control methods (Kumar and Varaiya 1986). While some measurements in a traffic control system will be highly accurate and reliable, many of them will not. Moreover, intercommunications cannot be utterly reliable and always timely between various nodes in the geographically dispersed sensor/control system. The communications and control network will resemble the traffic network since they both are described as an interacting array of queues. Hence the performance of the traffic control system will be highly dependent on the performance of the communications network and the uncertainty of the measurements.

This will require a detailed analysis and partitioning of the parameter space that allows autonomous actions to be carried out in the distributed process. Mutually beneficial goals must be identified and developed to act as a guideline for communicating and collaborating between interconnected nodes. While some information passed between nodes may not be necessary to determine the required control actions in a single node, such data must be sufficient to ensure that nodes are controlling traffic in such a way as to be mutually beneficial across all the nodes. 


\section{COMMUNICATION TECHNOLOGY}

\subsection{COMMUNICATION}

A TFWAS system might include hundreds of sensors in an area ranging from a tenth of a mile up to several hundreds of miles. Raw or fused data from these sensors must be communicated to the processor that deduces the traffic flow characteristics. In addition to the need for localized communications within a TFWAS system, it will also be necessary to provide longer distance communications between collaborating systems. The first section of this chapter presents some basic communications concepts. The following sections review current data communication techniques. The operational capabilities and limitations of each technique are discussed, as well as recommended applications.

\section{BASIC COMMUNICATIONS CONCEPTS}

This section presents some basic communications concepts and terminology. Knowledge of these concepts is necessary to fully understand the discussion of data communications techniques that follows. These concepts are arranged into four groups: The first group defines data types; the second group deals with data modulation techniques; the third group contains data transmission concepts; and the fourth group focuses specifically on digital data transmission concepts.

\subsection{Data Types}

Data communications systems can transmit two types of data, analog and digital. In an analog system, the signal can assume any intermediate value between the system's upper and lower limits. In a digital system, the signal may only assume discrete values between the system's upper and lower limits. Typical analog data are voice data; however, most data likely to be encountered in the TFWAS application will be digital. Digital data are preferred over analog for several reasons. Protocols and standards permit ease of coupling digital devices for data transmission. Digital signals are also inherently noise resistant because the informational content of the signal is not dependent on the signal's amplitude. Other advantages include easier maintenance of accuracy, lack of distortion induced by signal processing, and greater stability.

\subsubsection{Data Modulation Techniques}

Baseband transmission refers to data transmission without modulation. The process of modulation translates the message spectrum to another frequency spectrum. This is done to meet the overall system transmission requirements and to provide more efficient propagation. A modem which converts digital data to a modulated carrier waveform required by the communications link, and at the receiving end, another modem converts the data back to their original form. Three commonly used modulation techniques are amplitude modulation, frequency modulation, and phase modulation.

Amplitude modulation is one of the earliest modulation techniques. As the name indicates, the amplitude of the waveform is modulated by varying the strength of the carrier 
to transmit a signal. The simplest amplitude modulation technique is amplitude shift keying, where the presence or absence of a carrier denotes binary state. Amplitude modulation is susceptible to noise degradation and is therefore not recommended for sensitive applications.

Frequency modulation is a commonly used modulation technique that varies the frequency of the carrier signal to represent different signal values. Frequency shift keying refers to a binary frequency modulation technique where the system uses two different frequencies to indicate binary state. $A$ third frequency may be used to indicate an idle condition. Frequency modulation provides a better quality signal than amplitude modulation; however, the technique requires wider bandwidth.

Phase modulation and frequency modulation are similar. Phase modulation varies the phase of the carrier signal relative to a constant-phase reference to represent different signal values. The binary phase shift keying is one of the most efficient methods for transmitting digital data. Binary state is represented by two signals, $180^{\circ}$ out of phase. By using phase shifts less than $180^{\circ}$ apart, more than two states can be represented.

\subsection{Data transmission attributes}

One of the most important data transmission attributes is bandwidth. Bandwidth refers to the range of sinusoidal frequencies that can transmit through the communications link without unacceptable distortion or attenuation. The greater the bandwidth, the greater the amount of information that can pass along the link per unit time.

The transmission mode refers to the direction of data transfer that can take place on the communications channel between two stations. There are three transmission modes: simplex, half duplex, and full duplex. Simplex refers to a mode where data flows in one direction only. Since no two-way communication exists between the two stations, there is no receipt verification and no status reporting. Half-duplex transmission allows data flow in either direction but only in one direction at a time. Although this mode does provide the advantage of two-way communications, it requires additional control capabilities, and the process of reversing the data transmission direction can be time consuming. Full-duplex transmission allows data transmission in both directions simultaneously. This is typically done by using different frequencies for transmitting and receiving. Needless to say, full duplex is a more costly mode than either simplex or half duplex. In addition, if frequency multiplexing is used on a single channel, the one-way capacity is reduced.

Multiplexing allows the transmission of multiple data messages on a common transmission channel. There are three common multiplexing schemes: frequency-division multiplexing, timedivision multiplexing, and code-division multiplexing.

Frequency-division multiplexing basically divides the available transmission frequency range into smaller bands, each of which functions like a separate channel. Interference between the channels is possible with frequency-division multiplexing since the filters used to define the bands do not have sharp cutoffs. To minimize this problem, guard bands are placed between the channels. Another problem with this multiplexing technique is phase jitter, which is caused by the oscillators used to raise and lower the signal frequency. This multiplexing technique is gradually being phased out.

Time-division multiplexing divides the channel into time intervals. Each channel uses the same system bandwidth but is allocated a different time interval for transmission. Guard time is inserted between the channels to minimize any interference in adjacent channels. 
Code-division multiplexing is a technique developed by the Defense Department almost 50 years ago but which remains largely unproven for commercial communications. This technique uses a specified binary sequence for each channel to encode the data.

\subsubsection{Digital data transmission}

Digital data can be transmitted in one of two forms, parallel or serial. In parallel transmissions, all of the bits of the character are transmitted at once and require one data line for each bit. This technique is used extensively in computer-to-peripheral transmissions where the cable distances are short and high transmission speed is required. Because parallel transmissions require a line for each bit, this method becomes cost prohibitive as transmission distance increases. Serial transmission permits a signal to be transmitted using a single channel by transmitting the bits one at a time in sequence. Serial transmissions are more economical and more widely used than parallel.

Serial digital communications can occur synchronously or asynchronously. In an asynchronous system, start and stop bits are transmitted before and after each character to synchronize the receiver clock. The rate at which characters are sent varies. In synchronous communications systems, data are transmitted at a constant rate. The transmitter and the receiver must be synchronized, but because no start and stop bits are sent, this technique is more efficient than the asynchronous (Killen 1986).

\subsection{DATA TRANSMISSION TECHNIQUES}

Several data communications techniques are discussed in the following sections. These communications techniques can be broken down into two categories, landlines and wireless. Landlines include the low-bandwidth twisted pair, the higher bandwidth coaxial cable, and fiber optics. Data may also be communicated in a wireless fashion using radio waves. These waves may be transmitted from point to point using satellites, meteor bursts, or a series of terrestrial microwave transponders. Radio waves may also be transmitted to and from mobile transponders using satellites or terrestrial antennas. Narrowband or spread-spectrum technology can be used. Commercially provided services such as cellular or packet radio can be used to take advantage of existing communications infrastructures.

\subsubsection{Coaxial Cable}

Coaxial cable communications is a broadband technology that gained popularity in the 1970s and early to mid-1980s. Increasingly, however, existing coaxial cable systems are being upgraded to fiber optics, and planners for new installations are selecting fiber optics rather than coaxial. Coaxial is still widely used for a dedicated transport of video information between camera and the backbone communications hub (Gordon et al. 1993). Coaxial is appropriate for the transmission of data, voice, analog video, and digitized video.

\subsubsection{Basic system components and commercial systems}

The coaxial cable consists of a single inner conductor surrounded by an outer annular conductor that serves as a shield. The annular space between the conductors is filled with a dielectric material. A coaxial cable communications system would consist of the coaxial cable 
and repeater amplifiers. Frequency-division multiplexing and time-division multiplexing are typically used by a coaxial system.

There are several commercial coaxial cable systems. The analog L-type carrier system was introduced in the 1940s. The system has continued to evolve and today has the capacity of 10,800 frequency multiplexed voice circuits per cable. A digital coaxial cable system was introduced in the 1970 s. This system, called the T4M, has a capacity of 4032 time-divisionmultiplexed channels per cable and transmits data at a rate of $275 \mathrm{Mbits} / \mathrm{s}$. This transmission rate refers to the DS4 standard (Killen 1986).

\subsubsection{Recent technical advances}

Coaxial cable is a mature technology. More emphasis has been given recently to advances in other communication media such as fiber optics.

\subsubsection{Application notes}

While coaxial cable can also be used to transmit data and voice, it is most frequently used for the transmission of video signals. It has the capacity to transmit multiple video images over a single cable using standard equipment. It also provides ease of integration of video and data transmission (Rowe and Janoyan 1992).

\subsubsection{Disadvantages}

A major disadvantage of any landline system is that such a communications system requires hardware for the entire physical length of the link. A right-of-way hardened against all environmental hazards, such as electromagnetic interference, must be secured. Although the coaxial cable itself is extremely immune to noise and transients from external sources, connections are vulnerable. Because there is some radiation from coaxial cables, signal security cannot be assured as easily as for a fiber-optic communications system. A fiber-optic system also has a much lower bit error rate than coaxial.

Another disadvantage of coaxial cable is the requirement for numerous field-located amplifiers, with a typical spacing of about 1 mile, along the link. The required repeater spacing is disadvantageous compared with the 9- to 15-mile spacing required for a twisted-wire pair and up to 50-mile spacing for a fiber-optic link. Routine maintenance must be performed to keep the system balanced (Rowe and Janoyan 1992). Each repeater amplifies the signal rather than regenerating it; therefore, noise along with the incoming signal is amplified. This results in the accumulation of noise and limits the number of cascaded repeaters to approximately 60 (Gordon et al. 1993).

\subsubsection{Advantages}

Coaxial cable is extremely immune to noise and transients from external sources. The maturity of the coaxial cable technology is another advantage. Standards for data transmission exist, and standard equipment is readily available. The high bandwidth of coaxial is another advantage since it can be used for the transmission of video signals and has the capacity to transmit multiple video images over a single cable. The ease of integrating video and data is 
another advantage (Rowe and Janoyan 1992). Coaxial cable is capable of being used in a multidrop configuration; this means that more than one device shares a single communication line.

\subsubsection{Twisted Pair}

By far, the bulk of data communication has traditionally taken place over twisted-pair copper wire using low-speed asynchronous modems. Twisted pairs are suitable for the transmission of data, voice, and freeze-frame video. Twisted pairs do not have the capacity required for analog video transmission. It should be pointed out that with the emergence of intelligent traffic systems, designers are increasingly implementing other media such as fiber optics. Twisted-wire pairs simply cannot supply the bandwidth required for today's advanced highway applications.

\subsubsection{Basic system components and commercial systems}

The basic component of a twisted-wire pair is an insulated copper wire sized for the current-carrying requirement. Twisting a pair of copper conductors together reduces the electrical interference because the magnetic fields produced by each conductor tend to cancel out.

Several commercial twisted pair communications systems exist. The $\mathrm{N}$-type carrier system was introduced in the 1950s. These analog systems have evolved and are still in use today. The latest introduction was the N3, which was put into service in the 1960s. This system has a frequency multiplexed capacity of 24 voice circuits compared to the 12 circuits of the earlier N-type systems.

In the 1960s, Bell Systems introduced digital carriers. The T1 system has evolved and today operates at $1.544 \mathrm{Mbits} / \mathrm{s}$, known as the DS1 standard, and provides up to 24 combined data and voice channels that are time-division multiplexed. Repeaters are used every $6000 \mathrm{ft}$ for bit regeneration and retiming. The $\mathrm{T} 1$ system is economical for data transmission distances up to around 50 miles. The T1C system, which was also introduced in the 1960s, transmits data at 3.152 Mbits/s, known as the DS1C standard. It carries 48 time-division-multiplexed channels over standard wire. The T2 carrier system was introduced in the 1970s. It transmits data at $6.132 \mathrm{Mbits} / \mathrm{s}$, the DS2 standard, and carries 96 time-division-multiplexed channels over higher quality wire. The data transmission rate per channel for the T-series is $64 \mathrm{kbits} / \mathrm{s}$ (Killen 1986).

\subsubsection{Recent technical advances}

Twisted-pair copper wire is a mature technology. Most technical advances are improvements in other associated transmission equipment such as modems.

\subsubsection{Application notes}

Twisted-wire pairs likely will not provide sufficient bandwidth for most traffic surveillance applications. They do not have the capacity to transmit analog video but can be used to transmit freeze-frame video. Field equipment and local low-speed distribution networks may require only low-speed data transmission systems. These communication needs can effectively 
be provided by twisted-wire pairs. Twisted-wire pairs are also appropriate for some analog applications since no special signal processing is required for transmission.

Capital costs of installing an owned twisted-pair cable system is a substantial part of the system cost. An alternative is to take advantage of leased lines. Although the TFWAS operator would have to compete with other subscribers for use of the limited capacity of a leased system, there are several advantages to using a leased system. The difficulties of acquiring rights-of-way and maintaining the physical link are the responsibility of the commercial carrier.

\subsubsection{Disadvantages}

A major disadvantage of any landline communications system is that it requires hardware for the entire physical length of the link. A right-of-way hardened against all environmental hazards, such as electromagnetic interference, must be secured. The physical length of the link is also vulnerable. An aerial installation is subject to storm-induced damage such as falling trees. Construction activities pose a threat to buried cable.

Repeaters are required to transmit data over long distances. A repeater spacing of 9 to 15 miles is typical. Compared with fiber optic's up to 50-mile repeater spacing requirement, this is a disadvantage. The bit error rate of twisted-wire pair is also significantly higher than that of a fiber-optic system.

Although proper cable design and installation can hold noise to a low level, twisted-wire pair is susceptible to noise. A typical noise source is $60-\mathrm{Hz}$ power cables, which are often laid close to signal wire. While the $60-\mathrm{Hz}$ noise is normally filtered out, harmonics of the fundamental may cause interference. Impulse noise resulting from lightning and human sources such as automobile ignitions and electrical equipment also causes interference. Frequently, cables consisting of multiple pairs are used. Because such a cable communicates more than one signal at a time, some crosstalk can be experienced. The twisting of pairs does minimize this problem (Gordon et al. 1993). Because copper wires radiate, signal security cannot be assured as easily as for a fiber-optic communications system.

As mentioned above, twisted-wire pairs have a very limited capacity compared with other landline alternatives. This tends to limit their application. They are not suitable for the transmission of analog video and are limited to low-speed data transmission applications.

\subsubsection{Advantages}

One primary advantage of copper wires is that to transmit analog signals, no special signal processing is needed at either end. To hardwire digital signals more than a few hundred feet requires a modem at both ends. Repeater spacings for twisted-wire pairs range from 9 to 15 miles. This is an advantage over coaxial, which requires a repeater spacing of $\sim 1$ mile.

Like coaxial cable, copper wires can be used in a multidrop configuration. Twisted-wire pairs may be more cost-effective than fiber optic or coaxial for low-bandwidth transmissions over short runs.

The fact that the technology is mature and well understood is in itself an advantage. Standards for data transmission exist, and standard equipment is readily available. Installation of twisted-wire pair is less specialized compared with other landline media, and less sophisticated maintenance procedures are required. 


\subsubsection{Fiber Optics}

Over the past 20 years optical fiber transmission has become the transmission medium of choice for long-distance, high-speed, point-to-point communications (Rameswami 1993). Fiber optics can support the transmission of data, voice, analog video, and digitized video.

\subsubsection{Basic system components and commercial systems}

A fiber-optic transmission system consists of three basic components-a transmitter, an optical fiber, and a receiver. The transmitter consists of the light source and associated drive circuitry. The transmitter receives an electric input signal and translates it into a modulated light signal. The optical fiber provides the link to the receiver and guides the light by total internal reflection at the boundary between the core and cladding of the fiber. The receiver consists of a photodetector, plus amplification and signal restoration circuitry.

Two fiber types are commonly used: the step-index fiber and the graded-index fiber. The step-index fiber is characterized by a uniform refractive index throughout the core. The core refractive index can be made to vary as a function of the radial distance from the center of the fiber. This type is called a graded-index fiber.

Both the step-index and graded-index fibers can further be broken down into single-mode and multimode classes. A single-mode fiber sustains only one mode of propagation, while a multimode fiber may sustain thousands of modes. Multimode fibers offer several advantages over single-mode. Because the core of multimode fibers is larger, it is easier to launch optical power into the fiber, and the light can be launched using a light-emitting diode (LED) source, whereas a single-mode fiber generally must be excited with laser diodes. The disadvantage with multimode fibers is intermodal dispersion. When an optical pulse is launched into a multimode fiber, its power is distributed over all or most of the modes. The modes that propagate with more internal reflections will naturally arrive at the fiber end later than the modes that reflect less. This causes the pulse to spread out over time as it travels down the fiber. This effect can be reduced by using graded-index fibers (Keiser 1983). The graded-index fiber exhibits a refractive index that decreases continuously with the radial distance from the center of the fiber. This causes a continual bending of the light ray toward the fiber's optical center (Killen 1986). There are also ways to reduce the number of modes in a step-index fiber. One could reduce the fiber size, increase the wavelength, or decrease the difference between the core and cladding indices.

The first commercial fiber-optic system, the FT3, was introduced in the 1980 s. It consists of 12 cabled fiber ribbons. Each ribbon contains 12 optical fibers. Each fiber carries up to 672 channels of digital time-division-multiplexed voice. This system transmits data at a rate of $45 \mathrm{Mbits} / \mathrm{s}$. The transmission standard applicable to this system is referred to as DS3.

In 1988, the Synchronous Optical Network standards were introduced. These standards allow higher capacity (48 DS3's or more) and longer transmission distances. Optical spans of up to 50 miles can be provided without repeaters.

\subsubsection{Recent technical advances}

More than 2 million miles of fiber have been installed in the United States by the major telephone companies. About $54 \%$ of the fiber is being used today. Most of the links operate at data rates between 45 and $560 \mathrm{Mbits} / \mathrm{s}$, and several 1.7-Gbit/s systems are now available for commercial applications (Rameswami 1993). 
Much effort over the past 10 years has been in the development of high-bandwidth fibers. Single-mode fibers are available with bit-rate-times-distance products of $400 \mathrm{Mbits} / \mathrm{s}-\mathrm{km}$. Graded-index fibers have exhibited above $1 \mathrm{Gbit} / \mathrm{s}-\mathrm{km}$ (Sandbank 1980).

Future effort will likely be in the development of associated electronics. The use of fiber in the networks of the future will provide several hundred thousand users with access to a network with a capacity on the order of terabits per second. While the bandwidth is certainly available with commercial fibers, associated electronics create a bottleneck since they cannot sustain the higher data rates (Rameswami 1993).

\subsubsection{Application notes}

A fiber has nearly unlimited bandwidth compared to copper wire. Expansion can generally be accommodated by simply upgrading the transmission equipment connected to the fiber. If future growth is anticipated, fiber optics should be considered over other wired media (Komanecky and Minior 1992).

Fiber optics is used extensively for the transmission of data and voice. While video can be transmitted over a fiber-optic link, there is a lack of standardization for video transmission.

Since optical fiber transmission systems are naturally immune to electromagnetic interference or radio frequency interference, they are appropriate for data transmission where such interference is present and would impede other transmission means (Sandbank 1980).

The cost of a fiber-optic system is governed by the performance limitations. Bandwidth and optical power are constraints that define the required repeater spacing. Maximizing the repeater spacing minimizes costs but results in more dispersion and attenuation (Killen 1986).

Recently, an analysis of communications media for the Los Angeles Smart Corridor Project was undertaken. Fiber optics was chosen for the backbone of the communications system. Fiber optics was chosen over twisted-wire pair because of a requirement to transmit video images. A cost analysis was performed comparing coaxial with fiber optics, indicating that the systems were essentially equivalent (Rowe and Janoyan 1992).

\subsubsection{Disadvantages}

Fiber-optic cables require more specialized installation procedures than traditional copper wire, and splicing is complex. Additionally, more sophisticated maintenance procedures are required than for copper systems.

Also, as with all landline systems, hardware is required for the entire physical length of the link, and a right-of-way must be secured. Although video can be transmitted on a fiberoptic link, the requirements are more complex, and there is a lack of standardization for video transmission.

\subsubsection{Advantages}

One of the primary advantages of a fiber-optics link is its high capacity (Rowe and Janoyan 1992). Single-mode fibers are available with bit-rate-times-distance products of 400 Mbits/s-km. Graded-index fibers have exhibited above $1 \mathrm{Gbit} / \mathrm{s}-\mathrm{km}$ (Sandbank 1980). Optical fiber transmission systems can also achieve incredibly low bit error rates $\left(10^{-9}\right)$ compared with copper wire $\left(10^{-5}\right)$ (Rameswami 1993).

Optical fiber communications systems require repeaters to transmit data for long distances. However, up to 50 miles between repeaters can be achieved. This compares 
favorably with other landline systems, which require repeater spacings anywhere from about 1 mile for coaxial to 15 miles for twisted-wire pair.

Considering the high-bandwidth capability, for a given transmission requirement, considerable volume savings over copper wire can be realized. This is important if the use of existing underground cable duct space is under consideration. Optical cables are also less dense compared to copper cables and therefore weigh less. This weight savings is an important consideration for aerial cable installations (Sandbank 1980).

Optical fibers are inherently electrical insulators. Large potential differences between ends of the cable can be tolerated without protective circuitry. There are no arcing or spark hazards from short circuits or abrasion (Sandbank 1980).

The security of an optical fiber system is more difficult to breach than that of a metallic cable system. The fibers themselves do not radiate, and with careful attention to the design of the waveguide, the splices, the connectors, and cable manufacture, a relatively secure system can be attained. Optical fiber transmission systems are naturally immune to electromagnetic interference or radio-frequency interference (Sandbank 1980).

\subsection{Meteor Burst Communications}

Meteor burst communications provide a cost-effective alternative to satellites and terrestrial line-of-sight means to propagate radio waves. Meteor burst communications rely on the billions of small meteorites ranging from the size of a pea to the size of a grain of sand which enter the earth's atmosphere every day. As these particles travel, they ionize the air around them, leaving a trail of ionized particles. These ionized particles are capable of reflecting radio signals (Anderson 1992).

\subsubsection{Basic system components and commercial systems}

The meteor burst communications system basically consists of a master station and a network of remote stations. The master station sends out a continuous coded signal in the 40to $50-\mathrm{MHz}$ region. When a meteor appears in the proper location, a portion of the signal is reflected to a remote station. The signal triggers the remote station to transmit the data stored in its buffer. Information can be transmitted back and forth between the remote station and the master station until diffusion reduces the electron density in the trail to a value too low to sustain reflection (Anderson 1992).

A currently available system would consist of a master station that serves as the central data collection point and also provides the remote control of the remote stations. One master station can interface with and control several remote stations. The current state-of-the-art remote station can accept inputs from 32 analog/digital sensors and has a storage capacity of 1000 12-bit words.

\subsubsection{Recent technical advances}

Research began in the 1970s, and the principle has successfully been implemented for large-scale remote data acquisition networks. Some networks implemented in the United States have been in operation since the early 1980 s.

Most of the recent technological advances in meteor burst have been targeted to improve throughput, which has improved from 50 to 5000 bits/s in the last couple of years, and to 
incorporate voice communications, which are currently in the planning and demonstration phases.

Many improvements have resulted from general advances in electronics. This has led to the design and use of remote units incorporating advanced microprocessors, noise blanking circuitry, and advanced modulation techniques. The use of microprocessors also permits programming in high-level languages, data compression techniques, error correction coding, and more complex protocols.

\subsubsection{Application notes}

Meteor burst is suitable for transmission of sensor data readout, coded messages of up to several hundred characters, test messages of a few words, or long messages achieved by splicing together the transmissions of successive bursts. Because transmissions occur in bursts, this form of communication is not suitable for real-time analog voice transmission. Data rates as high as $128 \mathrm{kbits} / \mathrm{s}$ are achievable (Adarsh 1992).

Meteor burst is particularly suited for areas with harsh terrain where mountain ranges block satellite coverage into deep valleys and where installation and maintenance of conventional terrestrial communication links would be difficult.

Meteor burst is also appropriate for remote areas where traditional communications infrastructure may not exist and where power may not be available. The system transmitter for meteor-based communications requires around $100 \mathrm{~W}$ to transmit but has a duty cycle of $0.1 \%$. Therefore, the power can usually be supplied by solar cells. This is an advantage over satellite systems, which require too much power for remote telemetry (Adarsh 1992).

The distance between the master station and the remote station can range from 0 to about 1200 miles, depending on the antennas used. However, currently available equipment is inaccurate at short ranges of up to around 200 miles. Therefore, for a range of 0 to 200 miles, other communication modes such as line-of-sight ground wave should be considered. Meteor burst is most accurate for a range of $\sim 500$ to 900 miles (Anderson 1992).

\subsubsection{Disadvantages}

Typically, the ionized trail lasts only a few hundred milliseconds. The meteor burst communications system must wait for a suitably located meteor to enter the earth's atmosphere. The wait time ranges from a few seconds to a few minutes depending on the time of day and time of year. Because of this waiting time, meteor burst is best suited to applications that do not require real-time data transmission or rapid system response to system failure modes.

Ambient radio frequency from power and telephone lines, heavy equipment, etc., interferes with the receiving station's detection of signals from the transmitting station. The ambient radio-frequency noise around the receiver must be less than $-112 \mathrm{dBm}$ (Cerocke 1992). Because radio-frequency communications systems rely on electromagnetic radiation, both real and perceived biological hazards exist.

\subsubsection{Advantages}

A conventional line-of-sight radio system relies on a series of links to transmit further than the line-of-sight. The failure of a single transponder impairs the entire network. The meteor burst system does not rely on such a series (Cerocke 1992). 
Although transmitting and receiving equipment is required to transmit data over any length, since there are no costs for materials, installation, maintenance, or right-of-way for an interconnecting cable, a radio-frequency link can be significantly cheaper than a fiber-optic or copper link. To be cost competitive, the link must be long enough that the cost savings on the cable offsets the cost of the receivers and transmitters.

The meteor burst system is difficult to jam, compared to a satellite system. A satellite in a fixed spot in geostationary orbit can be jammed by a stationary jamming beacon. Meteors are random, and huge areas would have to be covered by a jamming system (Adarsh 1992).

Rain, snow, and other adverse weather conditions do not significantly attenuate signals at the frequency range used for meteor burst transmissions.

Another obvious advantage of meteor burst over satellite is that it is cheaper. The meteor burst transmitter/receivers are about half as expensive as the ground-based transmitters/receivers for satellites. Tariffs for use of a satellite link channel may be as high as several thousand dollars per month, whereas meteors are free.

\subsubsection{Terrestrial Microwave}

Microwave refers to signals in the radio-frequency range above $900 \mathrm{MHz}$. Terrestrial microwave links operate in the $928-\mathrm{MHz}$ to $40-\mathrm{GHz}$ bands. Microwave signals cannot bend over the horizon; thus, they travel through the atmosphere along a line-of-sight path and require terrestrial transmitting and receiving antennas to travel over the horizon. Terrestrial microwave is suitable for the transmission of data, voice, analog video, and digitized video.

\subsubsection{Basic system components and commercial systems}

Several microwave transmission facilities are in operation today. Both analog and digital trunk links exist. The TD2 and the TD3 operate over the 4-GHz common carrier band. The $\mathrm{Tl}$ system is a short-haul system that operates in the $11-\mathrm{GHz}$ band, and the $\mathrm{Th}$ is another short-haul system operating in the $6-\mathrm{GHz}$ band. A typical data transmission rate for a $6-\mathrm{GHz}$ digital microwave radio link is 140 Mbits/s (Killen 1986).

\subsubsection{Application notes}

Microwave may be applicable for data transmission over a several-hundred-mile range and would also be appropriate to use to span a natural barrier or to provide communications between points where rights-of-way are not available.

Since the operating frequency can significantly impact the performance of the system, the planner should carefully evaluate the available frequencies before selecting this method. In crowded urban areas, channels may not be available. The planner should also evaluate whether a TFWAS-owned terrestrial link or a commercially owned communications system should be used. The advantage of an owned, dedicated communications system is that the system can be optimized for the TFWAS application, and the TFWAS operator does not have to compete with other users for the available capacity. However, an owned system would have to be purchased, installed, and maintained. Another disadvantage of an owned system is that considerations such as obtaining frequency allocations and compliance with regulations would be the responsibility of the TFWAS operator.

An application of microwave communications proposed extensively for traffic applications is the use of roadside communications units called beacons. The beacon is a 
transmitter/receiver located along the roadside. It provides intermittent, two-way, digital, highspeed communications with automobiles. A system being developed by the Japanese, the Road Automobile Communication System, provides dynamic traffic information and can provide such information as automatic vehicle identification and automatic monitoring of a particular vehicle (Adarsh 1992).

\subsubsection{Disadvantages}

Microwaves cannot bend around the horizon; thus, repeaters are required approximately every 50-100 miles depending on terrain and the elevation of antennas. The failure of a single transponder impairs the entire transmission network.

Radio-frequency links are strictly regulated. They either require licensing or strict compliance with Federal Communications Commission (FCC) regulations. They also require frequency allocation either from the FCC or from the National Telecommunications and Information Administration (NTIA). These frequency allocations are difficult to obtain because the radio spectrum is scarce and an economically valuable resource. Above $11 \mathrm{GHz}$, atmospheric effects become more pronounced (Gordon et al. 1993). Because radio-frequency communications systems rely on electromagnetic radiation, both real and perceived biological hazards exist.

\subsubsection{Advantages}

Although transmitting and receiving equipment is required to transmit data over any length, since there are no costs for materials, installation, maintenance, or right-of-way for an interconnecting cable, a radio-frequency link can be significantly cheaper than a fiber-optic or copper link. To be cost competitive, the link must be long enough that the cost savings on the cable offset the cost of the receivers and transmitters.

\subsubsection{Satellite}

Satellite communication relies on a satellite to receive and retransmit radio waves. Satellite communications could be used to transmit voice, data, and video.

\subsubsection{Basic system components and commercial systems}

Geostationary satellites are located on the geostationary arc $35,900 \mathrm{~km}$ above the earth and operate above $1 \mathrm{GHz}$. A typical data rate is $20 \mathrm{kbits} / \mathrm{s}$. Three categories of communications channels are available to the user: store-and-forward packet data channels, interactive packet data channels, and circuit switched channels. Store-and-forward packet channels are the easiest to implement. They allow the transmission of small packets of user data with a delivery time delay that can be up to several minutes. Interactive packet data channels should be used where this delay is unacceptable. Circuit switched services are required for the transmission of real-time voice communications or large amounts of data such as for file transfers (Lodge 1991). 


\subsubsection{Recent technical advances}

Much of the growth in satellite communications is to fulfill the increasing demand for mobile communications. Satellites in low earth orbit $(800-1300 \mathrm{~km}$ above the earth) are proposed or existing for mobile communications needs.

One type of satellite system would operate in the 1- to $3-\mathrm{GHz}$ range. This system would provide traditional voice and medium- to high-speed data services up to a few megabits per second and mobile personal communications. The other major type would provide low-cost and low-data-rate two-way digital communications of up to $10 \mathrm{kbits} / \mathrm{s}$ to mobile terminals in the very high frequency (vhf) band below $400 \mathrm{MHz}$. Some advantages of low earth orbit satellites is that they are cheaper to launch, and because the signal does not have to travel as far as to a geostationary satellite, the path losses are less and the propagation delay is less. On the downside, because of its lower orbit, many more satellites are required to cover an area. It has been estimated that it will be the late 1990 s before these systems are fully operational (Reinhart 1992).

Compared with the satellites of today, future satellites will perform much more on-board signal processing. The signal processing of current satellites is limited to filtering, amplifying, and frequency translation. Future satellites will incorporate power conservation schemes and will be capable of on-board switching. On-board switching will permit intersatellite links and switching between beams on a signal-by-signal basis (Lodge 1991).

\subsubsection{Application notes}

Satellite communication is indispensable for continuous high-bandwidth communication over long distances of several thousand miles or around the world. Satellite communications are also appropriate for applications when portions of a shared database are stored at different sites hundreds or thousands of miles apart (Killen 1986).

Whereas other methods would certainly prove more cost-effective for communications within a TFWAS system, satellite may find application for communications among TFWAS systems.

\subsubsection{Disadvantages}

If data are only sent intermittently, renting a full-time dedicated satellite channel is not cost-effective. Rental of an earth station may be on the order of tens of thousands of dollars, and tariffs for a duplex channel usually start at around $\$ 2000$ per month (Killen 1986). Because radio-frequency communications systems rely on electromagnetic radiation, both real and perceived biological hazards exist.

Geostationary satellites can be easily jammed by a stationary jamming beam. Signal security cannot be assured as readily as for a meteor burst system.

\subsubsection{Advantages}

Although transmitting and receiving equipment and channel rental are expensive, since there are no costs for materials, installation, maintenance, or right-of-way for an interconnecting cable, a satellite link can be significantly cheaper than a fiber-optic or copper link. To be cost competitive, the link must be long enough that the cost savings on the cable or lease of the cable offsets the cost of the receivers and transmitters. One of the most 
important advantages of satellite communications is that the cost of data transmission does not increase with distance. As with other leased wireless techniques, considerations such as obtaining frequency allocation are the responsibility of the commercial carrier.

\subsubsection{Cellular Radio}

The existing cellular infrastructure, allocated the radio-frequency band from 806 to $902 \mathrm{MHz}$, presents one more method that can be used to transmit data. Although the cellular system was designed for voice communications, data can be transmitted using a cellular modem.

Cellular radio grew out of an idea developed at AT\&T Bell Laboratories in the late 1960s. They coupled the idea of frequency reuse of contiguous radio cells using analog FM technology with modern digital switching to allow mobility between the cells. A major feature of cellular is the use of low-powered transmitters to minimize interference between cells. The first cellular system was put into operation in Chicago in 1983 (Erdman 1993).

\subsubsection{Basic system components and commercial systems}

The cellular system consists of a mobile terminal that communicates with the radio base station that is nearest to it. Areas are divided into multiple contiguous zones referred to as cells. Each cell contains a base station that communicates with the active users in the cell.

Cellular radio developed from the analog Advanced Mobile Phone System (AMPS), the U.S. cellular phone standard mandated by the FCC. Today, cellular radio has four separate technology standards, two of which are analog and two of which are digital, and no direction from the FCC. If the cellular industry does not settle for a single digital standard, the domestic market will fracture among the competing technologies. This will result in increased equipment cost and decreased interoperability among systems (Erdman 1993).

\subsubsection{Recent technical advances}

The cellular industry is moving quickly to replace the low-capacity FM analog systems with digital. Digital systems have been advocated for better performance in a wireless environment. Channel coding, interleavening, and other digital techniques provide additional robustness for radio channels affected by shadowing, fading, and other forms of perturbation (Weissman et al. 1993).

The first digital standard to be recognized is based on time-division multiple access, offering roughly three times capacity improvement. This was implemented in 1986. However, some in industry decided to test a technology developed by the Defense Department, the spread-spectrum code-division multiple access.

A growing number of cellular providers are using cellular digital packet data protocols. This permits the transmission of data using the existing cellular phone spectrum by finding and using idle portions of bandwidth not being used for voice transmissions (Flanagan 1994). Cellular providers have also been evaluating the possibility of transmitting packet data in the guard bands between standard analog cellular channels. This differs from cellular digital packet data protocols, which would transmit packetized data across idle standard channels (Weissman et al. 1993). Transmission speeds by wireless are limited. Burst speeds can run as high as $19.2 \mathrm{kbits} / \mathrm{s}$, but when the transmission is more than a few packets, idle time between bursts can reduce the effective rate down to $1200 \mathrm{bits} / \mathrm{s}$. Cellular packet providers typically 
charge on the order of 50\%/packet, whereas cellular operators charge an average of $75 \mathrm{c} / \mathrm{min}$ for traditional cellular service (Flanagan 1994).

\subsubsection{Application notes}

Cellular radio will be important for mobility and for quick setups. Cellular technology may also find application where other wireless techniques are inappropriate and where rightsof-way cannot be obtained to route landlines. Cellular radio is also an appropriate and easily implemented means to communicate with vehicles.

The cellular infrastructure is being utilized for a traffic application in Japan. The Advanced Mobile Traffic Information and Communication System transmits information from a traffic control center to an in-vehicle navigation system using a two-way digital cellular communication system. The system provides real-time information of traffic conditions, weather and accident warnings, parking space availability, etc. The communications system transmits data at a rate of 9600 bits/s using $900-\mathrm{MHz}$ band frequencies. This project was initiated in 1987, with pilot experiments started in 1988 (Kawashima 1989).

\subsubsection{Disadvantages}

Wireless technology has experienced rapid growth over the past 10 years. In large, urban cellular markets, this widespread acceptance of cellular technology has resulted in capacity problems (Erdman 1993). While cellular technology has grown, the development of standards and regulatory direction have not kept up with the growth. It appears that the cellular industry may implement two digital standards that are incompatible with each other. This will result in more expensive equipment and lack of interoperability (Erdman 1993). Because radio-frequency communications systems rely on electromagnetic radiation, both real and perceived biological hazards exist.

Several conditions exist for cellular channels which make data transmission difficult. One fundamental problem is the handoff when a mobile station is transferred to a new base station in the adjoining cell. At handoff, the mobile station sends control information in a "blank and burst" mode. It temporarily mutes the transmission of user information. This hardly ever affects voice communication, but for data transmission, this can result in lost information. Similar disruptions can occur from channel interferences such as fading, attenuation, and path blockage. To contend with the degraded channel environment, error correction protocols can be used to maintain data integrity, but with a sacrifice in transmission speed (Weissman et al. 1993). Another transmission problem may involve the lack of signal security. Transmissions can easily be intercepted.

It may take up to $30 \mathrm{~s}$ for a cellular modem to set up a call and synchronize. Therefore, for real-time data transmission, the circuit would have to be held for the duration of possible data transmission. This would result in unnecessary charges (Flanagan 1994). Long-distance communications rely on a network of communications links. The failure of one of the links might impair the transmission of data.

The U.S. cellular market is based on circuit switched network architecture with an analog air interface that is tailored to voice communications, not data. Without the use of data compression of the frequency modulation analog cellular channel, the transmission can accommodate only low data rates, as low as $4.8 \mathrm{kbits} / \mathrm{s}$ in a $30-\mathrm{kHz}$ channel (Weissman et al. 1993). 


\subsubsection{Advantages}

Since there are no costs for materials, installation, maintenance, or right-of-way for an interconnecting cable, a cellular link can be cost competitive with a fiber-optic or copper link.

A primary advantage in using a commercial system such as cellular radio over an owned wireless technique is that considerations such as obtaining frequency allocation, compliance with regulations, acquisition and operation of repeater stations, etc., are the responsibility of the commercial carrier.

\subsubsection{Packet Radio}

Packet radio operates in bands between 150 and $960 \mathrm{MHz}$ allocated for land mobile communications. Specifically, the bands are 150 to $156 \mathrm{MHz}, 157.5$ to $162 \mathrm{MHz}, 220$ to $222 \mathrm{MHz}, 450$ to $470 \mathrm{MHz}$, and 928 to $960 \mathrm{MHz}$ for private land mobile communications. The government has been allocated the bands of 162 to $174 \mathrm{MHz}$ and 406 to $420 \mathrm{MHz}$. Radio-frequency signals in these frequency bands can bend slightly, can reflect off of obstacles, and can penetrate buildings. Packet radio is intended primarily for the transmission of data.

\subsubsection{Basic system components and commercial systems}

Packet-data networks cover most metropolitan areas (Flanagan 1994). Like cellular radio, the mobile terminal communicates with the radio base station that is nearest to it. Areas are divided into multiple contiguous zones referred to as cells. Each cell contains a base station that communicates with the active users in the cell. The base stations are connected to local switches. The local switches are connected to regional switches, which are, in turn, connected to national switches. Packet radio uses frequency reuse like cellular telephony and also features a trunking technology that increases capacity by routing calls to the first available channel (Hogberg 1993).

\subsubsection{Recent technical advances}

Packet radio is a relatively new communications means.

\subsubsection{Application notes}

Packet radio may be suitable for portable, quick, temporary setups. Packet radio was designed for the transmission of data, and the packet approach promotes a structure supporting intelligent data protocols (Weissman et al. 1993).

\subsubsection{Disadvantages}

One of the biggest problems for packet radio data transmission is the lack of standardized interfaces (Flanagan 1994). Failure of the communications links that form the structure of the network might interrupt long-distance transmissions (Hogberg 1993). Because radio-frequency communications systems rely on electromagnetic radiation, both real and perceived biological hazards exist. Also, signal security cannot be assured as readily as with a spread-spectrum system. Transmissions can be readily intercepted. Packet radio is generally not cost-effective 
for transmitting long files or for continuous transmissions. Currently, packet radio is only available in major cities. The time delay in delivering the packet may also be an unacceptable attribute (Gordon et al. 1993).

\subsubsection{Advantages}

Unlike the analog cellular circuit switched network, which must hold the circuit for the duration of transmission, a packet switched system avoids the problems of radio fading by only sending packets of data from the mobile terminal to the network when the signal is strong enough (Hogberg 1993). There is no charge for radio spectrum when no data are being sent. The cellular system, in contrast, incurs charges whenever the transmitting modem is on, whether or not data are being transmitted (Flanagan 1994).

Packet radio makes more efficient use of radio spectrum and can handle up to 1000 subscribers per channel compared to the 30 subscribers per channel offered by cellular radio (Hogberg 1993).

Since there are no costs for materials, installation, maintenance, or right-of-way for an interconnecting cable, a packet radio link can be cost competitive with a fiber-optic or copper link.

Another advantage to using a commercial system for data communications is that considerations such as obtaining frequency allocations, compliance with regulations, acquisition and operation of repeater stations, etc., are the responsibility of the commercial carrier.

\subsubsection{Spread-Spectrum Radio}

The spread-spectrum radio technique was developed in the 1940 s by the military. It has been used extensively by the military because of its resistance to detection, interference, and jamming by the enemy. The technique has lately gained popularity for traffic applications. Spread spectrum would be applicable for data transfer and low-end digitized video. Data rates up to $300 \mathrm{kbits} / \mathrm{s}$ can be achieved with currently available equipment.

\subsubsection{Basic system components and commercial systems}

Spread spectrum applies code-division multiplexing. The transmitter in a spread-spectrum communications system spreads the signal over a wide range of frequencies and codes it with a unique code known to the receiver. The receiver decodes the message and returns the signal to its original frequency range.

\subsubsection{Disadvantages}

Spread spectrum is somewhat line-of-sight dependent. Because radio-frequency communications systems rely on electromagnetic radiation, both real and perceived biological hazards exist. The range of a spread-spectrum communications system is quite small, on the order of 6 miles or less. This limits its applications to localized communications. 


\subsubsection{Advantages}

Spread spectrum does not require licensing in certain frequency bands. While this is an advantage for normal operations, it is particularly advantageous during the development and testing phases of a project. Since there are no costs for materials, installation, maintenance, or right-of-way for an interconnecting cable, a spread-spectrum link can be cost competitive with a fiber-optic or copper link.

This technique is more secure than other radio communications. Additionally, the resistance to jamming and interference is a plus. Spread spectrum is more resistant to multipath interference than the other wireless techniques discussed. Another advantage of spread spectrum is that it works very well in environments with a high level of electromagnetic noise (Gordon et al. 1993).

The same frequency band can be used by several different networks in the same area because each network can be assigned a different unique code. Likewise, multiple receivers could be assigned the same code, and multiaccess requirements could be provided (Hockaday et al. 1994).

\subsubsection{Application notes}

A spread-spectrum communications system is being developed by the Cal Poly Transportation Research Group for the Advanced Transportation Management and Information System. The spread-spectrum system will support half-duplex vehicle to TMC communications. Some of the services planned include traveler advisory broadcasts, automatic vehicle identification, emergency communications, and management of roadside infrastructure such as ramp meters and changeable message signs (Hockaday et al. 1994).

\subsubsection{Other}

This section addresses some of the more exotic and lesser developed devices that communicate via ultrasonic, infrared, and submillimeter signals. Such devices are being used extensively for consumer electronics. Although they have not found significant application for general communications, they may be practical for communications to sensors and between sensors for remote and autonomous operations.

\subsubsection{Basic system components and commercial systems}

The devices discussed in this section transmit and receive single-pulse or coded information using ultrasonic, infrared, and submillimeter signals. Examples of existing applications include television remote controls, car alarms, remote door locks, supermarket price scanners, and automatic door openers.

\subsubsection{Recent technical advances}

These technologies are lesser developed, and most advances are for consumer electronics. 


\subsubsection{Application notes}

While not practical for data transmission, these communications means could be used to communicate to a sensor or for communications between sensors. A typical application would be to turn on or shut off a system, such as activating a repeater, turning multiple operations on or off, activating channels, or triggering a sensor to download data.

\subsubsection{Disadvantages}

The techniques indicated in this section are typically only useful for a short range of a couple of hundred feet and are line-of-sight dependent. Another disadvantage is that they have a very limited application, and it is unlikely that they could be used for data transmission.

\subsubsection{Advantages}

These devices operate at frequencies that do not require licensing. Because these are wireless techniques that do not rely on a physical link between systems, there are no costs associated with materials, installation, maintenance, and right-of-way for interconnecting cable.

\subsubsection{Conclusions}

A wide variety of communications techniques is available for use with TFWAS. The selection of the technique or techniques used for a communications need will likely be quite application specific. Many questions must be considered when making technique selections:

- What data rate and response time must the communications link support?

- What type of data (analog, digital, video) is to be transmitted?

- Will transmissions occur continuously in real-time, or is only intermittent service required?

- Over what distance must data be transmitted?

- Are rights-of-way available for landline installation?

- Are frequency allocations available for wireless communications?

- Are existing commercial communications infrastructures that would provide adequate communications services available?

- Is future expansion anticipated, and is the medium of choice easily expandable?

- What sources of interference exist?

- What operational life span is required?

- Is security of the signal from interception or interference a concern?

- What environmental and biological effects must be considered?

- Is redundancy required to ensure uninterrupted service?

- What type of interconnection scheme exists? Is the medium supporting a point-to-point communication of data, or is it part of a network of a distributed data base?

- What is the most cost-effective communications means for the specific application?

- What is the existing physical infrastructure within which the communications system must be integrated? 
The following tables address many of these issues. Table 3.1 provides a comparison of several data transmission characteristics for the communications media considered, such as type of information supported, bandwidth, and data rate. Tables 3.2 and 3.3 contain cost information. It should be stressed that the cost for a communications channel can vary greatly from region to region, and the cost figures provided in the tables should be used for rough comparison purposes only.

Table 3.1. Comparison of the data transmission characteristics for the various media considered

\begin{tabular}{|c|c|c|c|c|}
\hline Technology & $\begin{array}{c}\text { Type of } \\
\text { information } \\
\text { supported }\end{array}$ & $\begin{array}{l}\text { Distance } \\
\text { between } \\
\text { repeaters }\end{array}$ & Bandwidth & Data rate \\
\hline Coaxial & $\begin{array}{l}\text { Data, voice, analog } \\
\text { video, digitized } \\
\text { video }\end{array}$ & Up to $1 \mathrm{~km}$ & $\begin{array}{l}350 \mathrm{MHz} \text { per cable } \\
\text { typically subdivided } \\
\text { into } 50 \text { - to } 100-\mathrm{kHz} \\
\text { channels }\end{array}$ & $\begin{array}{l}275 \text { Mbits/s, modems } \\
\text { typically support } \\
1200 \text { bits/s to } 10 \text { Mbits/s }\end{array}$ \\
\hline $\begin{array}{l}\text { Twisted-wire } \\
\text { pair }\end{array}$ & $\begin{array}{l}\text { Data, voice, freeze- } \\
\text { frame video }\end{array}$ & 9-15 miles & Up to $2.7 \mathrm{kHz}$ & $1200-3100 \mathrm{bits} / \mathrm{s}$ \\
\hline Fiber optic & $\begin{array}{l}\text { Data, voice, analog } \\
\text { video, digitized } \\
\text { video }\end{array}$ & Up to 50 miles & Up to $24 \mathrm{THz}$ per cable & $\begin{array}{l}\text { Typically } 45-565 \mathrm{Mbits} / \mathrm{s}, \\
\text { 1.7-Gbit/s systems are } \\
\text { available }\end{array}$ \\
\hline Meteor burst & Data & $\begin{array}{l}\text { Up to } \\
1200 \text { miles }\end{array}$ & 5-20 MHz & Up to $256 \mathrm{kbits} / \mathrm{s}$ \\
\hline $\begin{array}{l}\text { Terrestrial } \\
\text { microwave }\end{array}$ & $\begin{array}{l}\text { Data, voice, analog } \\
\text { video, digitized } \\
\text { video }\end{array}$ & Up to 50 miles & $12.5 \mathrm{kHz}-100 \mathrm{MHz}$ & $100 \mathrm{Mbits} / \mathrm{s}$ \\
\hline Satellite & $\begin{array}{l}\text { Data, voice, analog } \\
\text { video, digitized } \\
\text { video }\end{array}$ & $\begin{array}{l}\text { Up to } \\
3000 \text { miles }\end{array}$ & $7.5 \mathrm{kHz}-48 \mathrm{MHz}$ & $\begin{array}{l}10 \mathrm{kbits} / \mathrm{s} \text { up to a couple } \\
\mathrm{Mbits} / \mathrm{s}\end{array}$ \\
\hline Cellular & Data, voice & Up to 20 miles & Typically $30 \mathrm{kHz}$ & $1.2-14.4 \mathrm{kbits} / \mathrm{s}$ \\
\hline Packet radio & Data & Up to 20 miles & Typically $25 \mathrm{kHz}$ & $\begin{array}{l}4.8 \text { kbits/s with } 19.2 \text { kbits/s } \\
\text { planned }\end{array}$ \\
\hline $\begin{array}{l}\text { Spread } \\
\text { spectrum }\end{array}$ & $\begin{array}{l}\text { Data, low-end } \\
\text { digitized video }\end{array}$ & Up to 6 miles & Varies, $100 \mathrm{Mhz}$ typical & $\begin{array}{l}\text { Up to } 300 \mathrm{kbits} / \mathrm{s} \text {, some } \\
\text { modems may be limited } \\
\text { to } 9.6 \mathrm{kbits} / \mathrm{s}\end{array}$ \\
\hline
\end{tabular}


Table 3.2 Cost comparison of the owned communications media considered

\begin{tabular}{|c|c|c|}
\hline \multirow{2}{*}{ Medium } & \multicolumn{2}{|r|}{ Cost } \\
\hline & Equipment purchase & Installation \\
\hline Owned twisted pair & $\begin{array}{l}\text { Cost of wire varies according to wire } \\
\text { gauge and bundle size. }\end{array}$ & $\begin{array}{l}\text { Installation cost is generally a large percentage } \\
\text { of the total system cost. The installation cost } \\
\text { varies significantly with region and terrain. }\end{array}$ \\
\hline Owned coaxial & Cost of wire varies. & $\begin{array}{l}\text { Installation cost is generally a large percentage } \\
\text { of the total system cost. The installation cost } \\
\text { varies significantly with region and terrain. }\end{array}$ \\
\hline Owned fiber optic & $\begin{array}{l}\text { Cost of cable varies according to } \\
\text { performance requirements. }\end{array}$ & $\begin{array}{l}\text { Installation cost is generally a large percentage } \\
\text { of the total system cost. The installation cost } \\
\text { varies significantly with region and terrain. }\end{array}$ \\
\hline Meteor burst & $\begin{array}{l}\$ 10,000 \text { per remote station; } \$ 100,000 \\
\text { to } \$ 300,000 \text { for a master station. }\end{array}$ & $\begin{array}{l}\text { The installation cost varies depending on } \\
\text { several variables such as terrain features } \\
\text { which impact the ease of installation. }\end{array}$ \\
\hline $\begin{array}{l}\text { Terrestrial } \\
\text { microwave }\end{array}$ & $\begin{array}{l}\$ 10,000 \text { per transmitter/receiver. } \\
\text { Tower rental can range from } \\
\text { \$100/month to several thousand } \\
\text { dollars per month. }\end{array}$ & $\begin{array}{l}\text { The cost for antenna installation depends on } \\
\text { several variables such as required spacing } \\
\text { and beights of antennas, availability of } \\
\text { towers for lease, and terrain features that } \\
\text { impact ease of installation. }\end{array}$ \\
\hline Spread spectrum & $\$ 3000$ per field unit & $\begin{array}{l}\text { Installation costs are difficult to estimate } \\
\text { because they vary as a function of Jocation } \\
\text { and terrain. }\end{array}$ \\
\hline
\end{tabular}

Table 3.3. Cost comparison of leased communications media

\begin{tabular}{|c|c|c|c|c|}
\hline \multirow[b]{2}{*}{ Media } & \multicolumn{4}{|c|}{ Cost/Channel } \\
\hline & $\begin{array}{l}\text { Initial equipment } \\
\text { purchase }\end{array}$ & Connection fee & Monthly service fee & Usage billed \\
\hline $\begin{array}{l}\text { Leased twisted-wire } \\
\text { pair }\end{array}$ & & Up to $\$ 500$ & $\begin{array}{l}\text { Up to } \$ 350 \text { local + up to } \\
\$ 10 / \text { mile for terminations } \\
\text { in different exchanges }\end{array}$ & \\
\hline Satellite & $\begin{array}{l}\text { About } \$ 60,000 \text { for } \\
\text { an Inmarsat } \\
\text { earth station }\end{array}$ & & $\begin{array}{l}\$ 2400 \text { for a full-time, } \\
\text { dedicated channel }\end{array}$ & $\begin{array}{l}\text { Up to } S 0.25 / \mathrm{min} \\
\text { for a voice } \\
\text { grade channel }\end{array}$ \\
\hline Cellular & Up to $\$ 1200$ & Up to $\$ 100$ & Up to $\$ 50$ & Up to $\$ 0.55 / \mathrm{min}$ \\
\hline Packet radio & Up to $\$ 2000$ & Up to $\$ 50$ & About $\mathbf{\$ 3 0}$ & $\begin{array}{c}\text { About } \$ 0.125 \text { per } \\
512-B \text { message }\end{array}$ \\
\hline
\end{tabular}




\section{SENSOR TECHNOLOGIES OVERVIEW}

Although several technologies are capable of sensing various traffic parameters, no single technology stands out as the best provider of wide-area sensing capabilities for all settings and under all environmental conditions. By defining a wide-area surveillance architecture capable of supporting a multiple sensor technology suite, a robust system can be designed that can best assess the traffic conditions for a particular setting while withstanding the environmental variations peculiar to that geographic area. This design philosophy also enables an evolutionary approach to traffic detection and characterization which can take advantage of future technological advances in any sensor category.

The sensor technologies most commonly used in the United States today are the inductive loop detector, the magnetic detector, and the magnetometer (TDH 1990, p. 2). These traditional technologies rely primarily on sensors buried in the pavement. Many newer technologies under development rely on above-the-road or roadside mounting. Microwave radar detectors have been widely used for law enforcement but have been used on a very limited basis for traffic detection. Ultrasonic detectors are widely used in Japan (TDH 1990, p. 166). Infrared sensors have been used for pedestrian crosswalks in England but have not yet been extensively used for traffic detection (TDH 1990). Video image processing for traffic control has been the subject of several research programs and has shown much promise in several studies. Concepts such as the use of probe vehicles and Automatic Vehicle Identification (AVI) seek to take advantage of information or techniques which may not be practical or cost-effective to use solely in support of TFWAS but which may exist in support of other aspects of the IVHS such as toll collection or commercial vehicle operations.

Technical advances in the last 20 years have made some of these newer technologies much more feasible and reliable as replacements for traditional under-the-pavement sensors. A combination of several of these technologies may provide a basis for a more global sensing methodology that can provide a true wide-area surveillance. Each of these relevant technologies will be discussed and their advantages and disadvantages identified with respect to a wide-area surveillance capability. Many of these technologies also support other functions of IVHS, but these functions will not be discussed here.

Future development may change the outlook for beneficial use of these technologies. In addition, all of these technologies can benefit from and have benefitted from advances in electronics and signal processing.

\subsection{SENSOR CHARACTERISTICS AND THEIR IMPLICATIONS}

There are many factors to consider when selecting sensors for a TFWAS system. Sensors selected must be able to operate in the environmental extremes in which the surveillance system functions. Environmental problems include the more obvious temperature extremes, rain, snow, sleet, and fog. Additional environmental hazards include wind, vibrations, shadowing, electromagnetic interference, and surrounding infrastructure, both manmade and natural (Hughes 1994b). Sensors selected must have sensing ranges appropriate for the application and must be capable of providing necessary traffic parameters for the given application. Rather than design a universal TFWAS that would work in all environments, it is more cost-effective to have the system hardened only to those hazards it can reasonably be 
expected to face. For example, a system that can withstand severe winter conditions is a necessity in Minneapolis but a waste of money in Los Angeles.

The sensors must be suitable for installation within the highway infrastructure in a configuration that allows safe, reliable operation. Sensors should be selected to provide the most complete, accurate information about the traffic flow in the most cost-effective manner. Important cost considerations include sensor cost, installation cost, operating cost, maintenance cost, life expectancy, and repair and replacement costs. The possibility (real or perceived) of the sensor causing injury to humans or damage to the environment is another important consideration.

\subsubsection{Sensor Placement-Pavement-Based Versus Aboveground Technologies}

Many of the most common traditional traffic detectors are based on sensors buried in the pavement detecting a vehicle as it passes over the sensor. These buried sensors require expensive and time-consuming installation to break up the pavement, install and test the sensor, and repave over the sensor. Additionally, the installation process is extremely disruptive to traffic flows. Repair of the sensor naturally carries the same cost and time burden. The pavement around the sensor is also more vulnerable to future structural damage. A major advantage of buried sensors is a relative insensitivity to environmental changes or extremes. While there may be some concerns about accumulation of moisture, good design packaging and proper installation can usually mitigate this problem.

Many of the newer traffic sensors under development are based on an above-the-road or side-of-the-road mounting. These sensors can often be installed with minimal disruption to normal traffic flow. While these sensors require special training and knowledge for proper setup, installation, and maintenance, they will not require time-consuming and costly pavement breakup or repair. These exposed sensors must be hardened against environmental extremes such as heat, cold, wind, water, and ice. Additionally, the performance of these sensors is impaired to varying degrees by rain, fog, snow, etc.

\subsubsection{Active versus passive sensing}

Active sensors emit some form of field or wave into an area of interest (detection zone) and examine the properties of the signal scattered from objects in the area of interest. The changes in the transmitted and received signal can be used to detect vehicle presence, speed, etc. Active sensors can often easily provide measures of traffic but may raise serious concerns about long-range health implications of exposure to their emanations. An array of active devices used to widen the scope of detection must deal with the possibility of interference between the individual devices.

Passive sensors sense a field or wave emanating from a detection zone. These sensors look for changes in the properties of the emanation which can be associated with vehicle movement or presence. Purely passive devices emit no radiation and thus present no health hazard. A secondary bonus of passive operation is the reduced possibility of interference with other sensing devices or with nearby communication systems due to emanations.

\subsubsection{Point versus area sensing}

A point sensor can be defined as a sensing device whose activation zone is approximately the size of a single vehicle. Such a sensor cannot count, distinguish, or track two vehicles 
within its detection zone at the same time. An area sensor can be defined as a sensing device whose activation zone may exceed the size of a single vehicle and which can count and distinguish multiple vehicles in its activation zone simultaneously.

Point sensors may provide rather limited information but often require little signal processing for interpretation of event. Area sensors provide more comprehensive information but often require extensive and costly signal processing capabilities to produce usable data.

\subsubsection{Sensor system outputs-levels of data abstraction}

The ultimate goal of a TFWAS is to provide cognitive knowledge of the state of traffic to the traffic control system. Raw data from sensors must be distilled into information about the traffic flow. The degree of sophistication of the overall sensor system is determined by the types of sensors used, the processing and analyzing of data performed within the sensor system, and the requirements of the control system that receives and uses the sensor system output. Because of the widespread use of inductive loop and magnetic point detectors, present traffic control systems have been developed which require information extracted from strategically located point detectors. An evolution in sensor system intelligence allowing raw sensor data to be distilled into a sophisticated evaluation of traffic conditions will enable a revolution in the structure and approach of adaptive traffic control systems.

A discussion of the various sensor technologies that might be used for wide-area surveillance is not complete without discussing the ability to derive a desired level of information abstraction from the raw sensor data. A model of the levels of information shown in Fig. 4.1 is used to explain the various levels of abstraction derived from sensor system output.

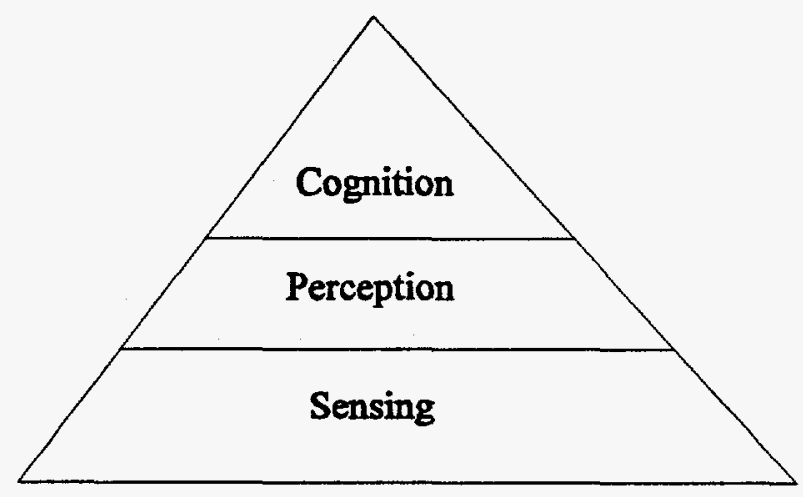

Fig. 4.1. Levels of information abstraction.

The lowest level of the triangle represents raw data from a single sensor. At this level the major objective of the sensor system is to improve signal usability and reduce noise. The correlation of these data to real traffic measurements and parameters must be performed by the control system. Control systems requiring information at this level would find the output of an inductive loop more meaningful than the output of a video camera because the data from an inductive loop would require significantly less complex computations to process. 
There are applications for sensors systems which provide only data. For instance, many simple, stand-alone intersection control systems may require information at this level.

The next level in the triangle, developing percepts about the traffic flow, involves increased processing of relevant parts of the data to extract measurements of some aspect of the signal. At this information stage, the machine perception process begins. The processing performed is probably based on a priori knowledge or expectations of manifestations of relevant phenomena to be detected and evaluated. This is primarily the level at which most video image processing systems operate. At this level, some machine interpretation of data is performed by the sensing system, and information about certain parameters is passed to the control system. The control system would be required to interpret this information and its relationship to traffic flow. While there is a great deal of variability and quality in the types of percepts available from different sensors, this is the level at which most higher level traffic detection technologies operate now.

Cognition, the apex of the triangle, is the result of a process of evaluating percepts. Cognition leads to an accurate mathematical summarization of the state of traffic flow. At this processing level, many percepts, including seemingly contradictory ones, are integrated to provide a consistent knowledge of the traffic flow. This combination of percepts from various sensors ensures robust, consistent, and reliable operation under a variety of environmental conditions. Additionally, the changes in this information over time are also taken into account in developing the overall picture of traffic flow. At this point, the sensing system has analyzed the data available from the sensor/sensors, has considered any a priori knowledge or expectations, and has applied both to provide a detailed interpretation/description of the present state of the sensed environment. This cognitive knowledge is then passed directly to the control system, relieving the control system of all aspects of the perception and cognition task. The level at which the sensing system provides information to the control system must be balanced with control system requirements and physical roadway complexity to ensure that traffic will flow optimally at the most reasonable cost.

With each increase in data abstraction available from the sensing system comes an increase in the computational requirements for the system, an increase in system complexity, an increase in system cost, and higher system reliability requirements. The increased utility gained from each higher step in the pyramid and the reduced burden on the control system must be weighed against these costs and requirements.

In summary, a lower level system produces raw sensor data. A more elaborate system produces some measures of traffic response through a process of machine perception. At a more mature level of technology, through machine cognition, knowledge about the traffic state can be derived. The overall process takes raw data, develops information, and produces a knowledge of the traffic flow. Present-day traffic surveillance systems do not yet meet this goal, but they do provide varying levels of information abstraction. Therefore, a discussion of various sensor technologies that can be used for the purpose of wide-area surveillance cannot truly be limited to the sensing elements alone but must cover the sensors in the context of applicable signal processing and computing frameworks and the types of information that can be distilled from the sensor data.

\section{TRAFFIC PARAMETER DEFINITIONS}

Prior to discussing the capabilities of various sensors to measure traffic parameters, a concise definition for these parameters must be provided. Some of these parameters are 
sensed directly, while others can be calculated based on data collected from the sensor/sensors.

The measures that might be directly sensed include speed, count, presence, and occupancy. "Speed" here is defined as the speed of an individual vehicle as it passes a specific point. Count refers to the number of vehicles that have passed a particular point. Presence is a determination of whether a vehicle is occupying a particular zone at the present time. Occupancy is the percent of time the detection zone of a detector is occupied by some vehicle.

Some of the aggregate parameters that have been found advantageous for traffic definition include volume, queue lengths, demand, time headway, throughput, link travel time, and slug velocity/acceleration. The volume or flow rate is a temporal measure defined as number of vehicles passing a specific point in a given period of time. Note that this definition is based on the current standard for point surveillance but could be adapted to an area definition. The demand flow rate on a facility is a measure of needed roadway access for a given set of conditions. Time headway is the "elapsed time between the passage of consecutive vehicles in the traffic stream." Throughput has been defined as "the vehiclemiles of travel carried by a given length of roadway for a given period of time." Density is the number of vehicles occupying a section of roadway at a particular time (Hughes 1994b).

Link travel time, the average time for a vehicle to traverse a link in the transportation network, is a very useful figure of merit both as a control parameter and as a planning tool. It can act as a measure of congestion to pinpoint trouble spots and to provide quick feedback to assess the effect of a control action. Link travel time permits comparison of performance of different links, identifies critical links, and serves as a quantitative measure of improvement due to new construction. Compared to a measure of delay, many researchers have found link travel time to be a much easier parameter to define, assess, and control.

Link travel times are calculated from flow or occupancy data. A surveillance detector provides a direct measure of flow, occupancy, or both. The Traffic Detector Handbook (TDH 1990, p. 328) defines occupancy as the percentage of time a detector is occupied. Identification of the function that deduces link travel time from point detector data is a difficult problem. All the present models are site specific. They consist in observing link travel times vs flow or occupancy for a given site, using the observed data to derive a regression model, and hoping that the model is a reasonably complete description of the site.

A slug velocity is the average velocity of a group of vehicles traveling in a cluster. Slug velocity and acceleration as functions of flow may become a useful figure of merit in future surveillance and control systems. This relationship can be obtained with a cellular automaton (Schadschneider and Schreckenberg 1993). A cellular automaton is a recursive replacement rule. It sees a system as a multidimensional array of cells in which the next state of a cell is a function of the present state of the cell and the present states of its nearest neighbors. A cellular automaton does not presume linearity of the functional relationship. Since the computation only involves nearest neighbors, the cellular automaton is conveniently scalable. The model is more general than the regression models used in conventional practice. Early results are encouraging.

An outgrowth aggregrate parameter for describing traffic conditions is incident detection, which is often tied into traffic surveillance and traffic quantification. Development of an automatic incident detection system initially involves the identification of traffic features that take values unique to incident conditions and the combination of the above features into some sort of algorithm (Cohen and Ketselidou 1992). Some sensors have already been developed to include such an incident detection function. 


\subsection{INDUCTIVE LOOP}

\subsubsection{Description and Implementation}

An inductive loop is an insulated loop wire that is installed under the surface of the road and acts as the inductive element in a tuned electric circuit. When a vehicle stops or passes over the loop, the inductance of the loop is decreased. This decrease is detected by electronics and transmitted to a controller unit to signal the presence of a vehicle. Traffic loops can provide an indication of vehicle presence, a running count of vehicles detected, and a measure of occupancy. By careful choice of loop characteristics, of loop location in roadway, and use of multiple loops, several traffic parameters can be deduced, including estimates of queue length, traffic volume, and speed (TDH 1990). These estimates require additional computational power and make assumptions about the traffic flow.

\subsection{Advantages and Disadvantages}

The major advantages of this detection technology is that it is a mature, well understood, and widely used device by present modern traffic engineers. Because the loops are installed under the pavement, they are not affected by normal variations in environmental conditions. The major disadvantages are its limited reporting capacity, its high installation cost, and its limited lifespan. Because the loop must be buried beneath the road surface, installation can be quite disruptive to traffic flow, and the road surface is weakened by the installation process. Reliability of loop detectors is often decreased by poor installation techniques. A study has estimated that at any given time approximately one-fourth of the loop detectors in New York City are out of order. This ratio seems consistent with reports in the United Kingdom (Dickinson and Wan 1990).

Loop detection is a fairly mature technology. Some recent improvements in loop technology are better protective packaging or improved digital circuitry for the loop output. A great deal of the present controls publications and research involve using data from loop detectors since it is the primary technology in use at this time in the United States.

As a TFWAS technology, loops have fairly limited capability. To derive an accurate description of the state of traffic over a large or highly complex area will require a large number of loops, a high installation cost, and high replacement costs. Even so, such a network of loops would be truly limited in its flexibility and its ability to derive many different types of information. A TFWAS system based primarily on inductive loops would not provide a good measure of traffic flow and would not even begin to provide the data needed for a cognitive description of traffic flow. Inductive loops are probably a necessary part of the sensor array needed for a full-featured TFWAS.

\subsection{MAGNETIC TECHNOLOGY}

\subsubsection{Description and Implementation}

Several variations of detector devices are embedded in the roadway and measure variations in the earth's magnetic field due to the passing of a vehicle. The two most commonly used magnetic detectors are the magnetometer and the magnetic detector. Magnetometers are cylindrical probes embedded vertically in the surface of the roadway. The 
presence or passage of a vehicle is detected by measuring the focusing effect of the earth's magnetic field that occurs when a vehicle is near the detector. Magnetometers can also be used for application such as looking for buried ordinance. Magnetic detectors are small, cylindrical probes that detect the passage of vehicles based on a change in the flux lines of the earth's magnetic field. These devices are installed horizontally in the roadway with the long axis perpendicular to the direction of travel. These devices are limited in application because they only work as long as the vehicle is in motion. These devices can be useful in areas not conducive to loop installation such as bridges (TDH 1990).

Primary factors affecting magnetometer reliability include probe burial depth, proper alignment and support in its placement, and moisture penetration.

\subsubsection{Advantages and Disadvantages}

Magnetic technology has some of the same disadvantages as inductive loops. There is a high installation expense since they must be installed within the pavement. In addition to the material and labor expense, this leads to traffic disruption caused by installation or maintenance work on the magnetic detector (TDH 1990). A number of detectors are required to cover a large section of roadway. These detectors are functional under all weather conditions. The use of an array of magnetometers mounted overhead or along a center divider in conjunction with sophisticated signal processing may yield multiple response zones for the detection of vehicle passage (Hughes 1994b).

Magnetometers can measure presence or passage of vehicles and thus infer a count, occupancy, and, with use of multiple detectors, speed or queue length. Passive magnetic detectors cannot detect a vehicle that is not moving or moving at an extremely low speed (a few miles per hour).

Like inductive loops, magnetic devices are limited in the type of information and true wide-area sensing they can perform. Magnetometers may perform better than loops at counting closely spaced vehicles and are very useful for detecting vehicles in locations where loops cannot be used, such as on bridges. As far as installation is concerned, magnetometers require less cutting of the pavement and can function longer in crumbly pavements than inductive loops.

\subsubsection{Present Research Efforts}

The Self-Powered Vehicle Detector developed with FHWA support is a self-contained magnetometer device with a transmitter that can broadcast passage or presence information to a remote receiver. This device has application as a temporary installation or where it can be mounted under bridges and viaducts (Hughes 1994b).

\subsection{MICROWAVE TECHNOLOGY}

\subsubsection{Description and Implementation}

In the description of microwave technology, both the traditional microwave radar range from 1 to $30 \mathrm{GHz}$ and the millimeter wave range, 30 to $100 \mathrm{GHz}$, are included. The FCC has allocated the frequency bands near $10.5,24.0$, and $34.0 \mathrm{GHz}$ to traffic applications (Hughes 1994b). Although microwave can be used to sense presence by aiming a microwave 
transmitter at a receiver on the opposite side of a detection zone and examining changes in receiver output, microwave radar generally uses a collocated transmitter and receiver and takes advantage of the Doppler effect to measure speed. These microwave systems transmit a beam of energy in the microwave frequency range into the roadway and detect changes in the reflected signal as it bounces off a vehicle.

The information-gathering capabilities of a microwave radar system rely largely on the characteristics of the transmission signal and the signal processing performed on the received signal. This waveform is selected based on the type of target, the environment, and the information to be extracted about the target (Brookner 1977). The most common radar signals consist of a microwave frequency carrier wave modulated by a square wave function whose duration is large relative to the carrier wavelength. This results in a narrowband signal with most of the energy about the carrier frequency. For other applications, continuous wave (CW) or frequency modulated continuous wave (FMCW) signals are used (Robinson 1967). The simplest continuous wave radar is used for detection and tracking of moving targets. A single sinusoid is transmitted, and the received signals are mixed with the transmitted carrier frequency. The Doppler shift determines the existence of the moving target and its velocity relative to the transmitter (Nathanson 1969).

If distance from a moving object is a desired measure as well as velocity, pulse delay or FMCW techniques can be used. The signal for a pulse delay system is a train of pulses that are samples of a single unmodulated sine wave. Pulse delay directly determines the transit time from the radar to the target and is typically used only for distances greater than $100 \mathrm{ft}$. This may limit its practicality for traffic applications. For FMCW a continuous wave signal is frequency modulated using a linear sawtooth or a sine wave. The use of a FMCW signal for determining distances involves converting the time interval between pulse transmission and reception to a frequency difference. The signal used for this method uses a linearly ramped frequency variance whose duration will exceed the time to transmit and receive the reflected signal (Schultz 1993). Microwave sensing systems are generally designed for an overhead or side firing mount for the primary function of vehicle detection and/or velocity measurement.

In summary, microwave sensing systems can sense presence, speed, and range and can calculate count, occupancy, and traffic density. Depending on the radar signal chosen and the beam width, multiple lanes of traffic can be monitored by a single sensor.

\subsubsection{Advantages and Disadvantages}

Primary factors affecting microwave operating capability are distance to the target, target reflectivity characteristics, and the medium through which the wave must propagate. Microwaves are attenuated in the atmosphere by gaseous absorption and water particulate scattering. Below $10 \mathrm{GHZ}$, the amount of attenuation due to atmospheric conditions increases with increasing frequency but is relatively small. For distances over a few miles, the attenuation is significant at the higher frequency range, 10-100GHz (Schultz 1993).

Microwaves maintain good penetration even in the presence of rain and fog and are the best overhead sensing technique under poor weather conditions. Microwave sensors also have dimensional tolerances proportional to wavelength for less difficult to fabricate parts. Microwave devices are overhead or side mount technologies, which may be less costly to install and maintain than more traditional road surface technologies.

Disadvantages of microwave sensors include the large physical size relative to infrared and optical systems, higher cost, and the limited spectrum available for automotive use 
(Bierman 1991). Additionally, the public perception of possible damage to people from exposure to microwaves is a major concern.

The primary technical problem of radar is the clutter problem-unwanted reflections from objects that are not vehicles that can cause false detections. The second primary problem with radar is multipath-waves that are picked up by the antenna after bouncing off several reflecting surfaces-also causing false detections.

Microwave radar detection systems can be vulnerable to jamming. Although any aboveground sensing technique may be vulnerable to sabotage, vandalism, etc., radars are vulnerable to intentional or coincidental frequency jamming. However, while a system can be designed to mitigate this vulnerability, it is probably not worth the additional cost involved.

Microwave devices have the capability of approaching, in a limited sense, a true widearea sensing tool. The ability to extract several different types of measures over a true area of roadway-not just point monitoring over a small, vehicle-sized portion of a lane-distinguish this technology as a good candidate for wide-area surveillance sensing. Additionally, the relative tolerance of microwave systems to inclement weather may give this technology a unique place in any wide-area surveillance scheme.

\subsubsection{Present Research Efforts}

A system using FMCW radar, Doppler radar, and signal processing techniques to obtain a signal "profile" that could be used to classify vehicles in a single lane into one of five categories with a 75\% accuracy has been developed (Roe and Hobson 1992).

Because of smaller antenna components needed for millimeter wave radar, it is widely being considered for vehicle installation as part of a collision avoidance system in Japan and Europe. Both human factors and use in such a dynamic environment make this a very difficult problem (Fukuhara and Kurami 1994).

Although most radar street traffic research has been centered around a fixed radar beam and the detection and speed of a vehicle/vehicles in the beam's path, the use of an imaging surveillance radar could truly revolutionize the capability of microwaves in the traffic surveillance field. With proper design, such a surveillance radar could monitor all vehicles over an extensive area, operate well under adverse weather conditions, be nondisruptive to traffic in the installation phase, and be cost-effective relative to the use of many point detectors.

The Massachusetts Institute of Technology Lincoln Laboratory has been conducting research under Federal Airation Administration (FAA) sponsorship at Boston's Logan Airport. The goal is to track the exact movments of all airplanes and vehicles on runways and taxiways with the specific objective of preventing collisions. The system looks at hundreds of targets and keeps track of each. This differs from TFWAS, which is concerned with the flow pattern, not individual movements and not specifically where to prevent collisions.

Lincoln Laboratory modified a commonly used marine radar and performed specialized signal processing to reduce clutter and eliminate data from areas that are not of interest. This system has proven to be highly reliable. Many of the techniques used for clutter and multipath reduction might be adapted and modified to meet the purpose of a street traffic area surveillance detector. (Andrews et al. 1994).

Thomson-CSF in conjunction with Societe des Autoroutes Paris-Rhin-Rhone have developed two millimeter wave radar systems. One system concentrates on measuring traffic parameters; the second system focuses on automatic incident detection (personal communication with Matthieu Pilissie du Rausas, Thomson-CSF, 1994). 


\subsection{ULTRASONIC TECHNOLOGY}

\subsubsection{Description and Implementation}

Ultrasonic sensors emit sound waves with frequencies above the audible level $(\sim 20 \mathrm{kHz}$ and greater) and detect these waves as they are scattered by the roadway and passing vehicles. Vehicle speed can be directly detected by sensing the Doppler frequency shift of the reflected sound wave from a continuous ultrasonic beam. Presence and range sensing can also be performed via a pulsed ultrasonic signal.

Ultrasonic sensors can measure passage, presence, and speed. They can deduce count, occupancy, and, with multiple detectors, queue length. Most ultrasonic detectors currently available only monitor one lane per sensor.

\subsubsection{Advantages and Disadvantages}

The ultrasonic spectrum has no allocation or regulation problems as microwave does. As with other overhead or side-mount sensors, ultrasonic sensors are minimally disruptive to traffic flow and the pavement during installation, maintenance, and repair. Since the emanations of ultrasonic detectors are not electromagnetic fields, it may be more acceptable than microwave from a standpoint of possible hazardous effects.

Disadvantages of ultrasonic sensors include an extremely short range, sensitivity to environmental effects, and limited ability to detect multiple vehicles in a single beam.

\subsubsection{Present Research Efforts}

Though not presently in common use in the United States, ultrasonic detectors are used extensively in Japan. The Japan Traffic Management Technical Association sponsored tests and analyses using ultrasonic sensors to estimate travel time for a section of roadway no longer than $2 \mathrm{~km}$. The test setup used downward-looking ultrasonic sensors to obtain a profile of a vehicle, one at the beginning of the stream and a second at the end. A three-lane section of roadway with only a few inflow and outflow accesses was chosen for the test. The entrance and exit times are monitored, the output profile and input profile with the highest correspondence are selected as a match, and the travel time is calculated (Sumi et al. 1989).

\subsection{PASSIVE ACOUSTICS}

\subsubsection{Description and Implementation}

As vehicles travel down a roadway, they produce audible noise. These sounds may be detected and used to determine a range of useful traffic parameters. The data collected by the acoustic sensing system, in the form of sound power level as a function of frequency, is processed through algorithms that result in an indication of vehicle presence. Use of multiple contiguous zones can provide an estimation of speed.

In one technique, the acoustic energy in a detection zone is monitored by several microphones mounted in an array. In the case of the AT\&T Smart Sonic TSS-1, 72 microphones make up the array. These arrays are mounted either aerially or to the side of a freeway or surface street (Hughes 1994b). A typical detection zone of an array is a lane 
with a range of 20 to $35 \mathrm{ft}$ and a $20^{\circ}$ beam pattern (Hughes 1993). However, the detection zone varies as a function of sensor aperture size, processing frequency band, and installation geometry (Hughes 1994b).

Passive acoustic arrays have been used to replace magnetic induction loops since they can provide a compatible contact closure to signal vehicle presence whenever sound energy rises above the detection threshold (Hughes 1994b). In more advanced applications, information of interest such as vehicle speed, count, or vehicle class can be deduced.

\subsubsection{Advantages and Disadvantages}

Acoustic systems are relatively easy to install and maintain. They can be purchased for less than $\$ 2000$ per detection zone and are very reliable. They are less susceptible to electromagnetic interference and adverse weather conditions than many other detector technologies. They are, however, susceptible to interference resulting from unpredictable acoustic energy. Predictable noise can generally be dealt with by configuring the filtering algorithms; however, noise sources that cannot be predicted may result in erroneous information (personal communication with Greg Pieper, AT\&T, 1994).

\subsubsection{Present Research Efforts}

Extensive research has been conducted on the analysis of acoustic signatures of onground vehicles (taxiing airplanes, motorcycles, etc.). Most of this has been conducted for drug enforcement and military covert operations and has not been published in the literature.

Oak Ridge National Laboratory (ORNL) has performed unclassified research in acoustic signature analysis for ground vehicles (Dress and Kercel 1994). Based on both Fourier and wavelet analysis, using a single microphone, it is possible to obtain an acoustic signature; perform real-time analysis; use the result to tell the speed, distance from microphone, and the class of the noise source; and to do so with a device that can be constructed for a few hundred dollars a copy when produced in quantity. This algorithm has been tested on airport sounds, highway sounds, and thunder. It can tell the difference; it does not confuse thunder with highway trucks. With further research, this system could easily be adapted into a classifying sensor for TFWAS and similar traffic monitoring applications.

Suppliers are currently working on systems that use sophisticated algorithms and neural networks to classify vehicles according to whether the vehicle is a car, a van, a pickup truck, or an 18-wheeler (Hughes 1994a).

\subsection{INFRARED TECHNOLOGY}

\subsubsection{Description and Implementation}

Passive longwave infrared detectors (also known as thermal radiation detectors) have been developed to monitor a section of the roadway and detect objects based on the infrared energy emitted by the object as a result of the object's temperature and emissivity. A car, motorcycle, or human will all have a thermal contrast with respect to the background of the road. Though originally these devices could only sense moving objects, signal processing has now provided these detectors with the capability to sense presence as well (Cima 1992). 
Active photoelectric sensors can be divided into three basic sensing modes. In direct sensing mode, an opaque object passes between the source of an infrared beam and its receiver. For reflex sensing, the beam's source and the receiver unit are side by side. $A$ retroreflector is placed opposite this arrangement to reflect the source's beam to the receiver unit. An object to be sensed will pass between the source/receiver and the retroreflector, breaking the path of the beam. Diffuse sensing is similar to reflex sensing except that the object to be detected has a reflective surface that takes the place of the retroreflector. Diffuse sensing is not practical for traffic purposes (Garner, Lee, and Huang 1990).

Presently marketed active detectors can provide the following traffic measures: presence, speed, volume, range, and vehicle classification. Presently marketed passive infrared detectors can provide volume and presence information.

\subsubsection{Advantages and Disadvantages}

Infrared detectors are vulnerable to dust, fog, mist, rain, and snow, which can scatter and attenuate their beam energy. However, infrared sensors are less sensitive to these effects than visible spectrum sensors. Sudden changes in lighting levels can cause false detections. Passive infrared detectors take advantage of naturally occurring thermal contrasts and so emit no potentially harmful electromagnetic energy. Passive infrared is one of the few detection technologies that can detect pedestrians or bicyclists as well.

Infrared detectors are generally used from an overhead or side-mounted configuration and thus avoid the installation costs, traffic disruptions, and physical deterioration of the pavement caused by in-road detectors. The narrow beam width of the infrared allows finely defined detection areas.

\subsubsection{Present Research Efforts}

One manufacturer has developed an active infrared sensor that has proven $\mathbf{9 9 . 4 \%}$ effective in detecting vehicles during tests in a municipal traffic environment. This sensor also measured vehicle speed to a $\pm 1-\mathrm{mph}$ accuracy up to a maximum of $50 \mathrm{mph}$. This sensor can also supply vehicle height profiles (Olson et al. 1994).

\subsection{VIDEO IMAGE PROCESSING}

\subsubsection{Description and Implementation}

Image processing involves extracting traffic parameters and/or vehicle information from individual images or a series of images. The imaging system that acquires these images may use a camera and lens system sensitive to visible light waves, to infrared waves, or both. While either setup has its unique properties and advantages, many of the problems and processing techniques used with images obtained from cameras responsive to visible light are similar to those used with images obtained with a passive infrared camera. Most video processing systems involve a camera system mounted in an overhead configuration with a field of view extending well beyond the immediate vicinity. Because a great deal of processing time is necessary to process possible traffic information for the entire digital image, most video image 
processing systems monitor only predefined areas of the image. These areas can be located and used to emulate the functionality of many inductive loops spread over several lanes without the installation expense and disruption to the roadway.

By using special image and signal processing techniques, several traffic parameters can be extracted from a video image processing system. These include presence, count, approximate speed, and vehicle classification. From these measurements, occupancy, volume, time headway, and queue lengths can be estimated. Several video image processors have incident detection capabilities as well (Hughes 1994b).

To reliably detect vehicles and measure traffic parameters, video image processing systems must be installed and programmed to overcome several major obstacles. Variable lighting and shading can cause false detections. The system must be capable of adapting to various types of backgrounds and to calibration and system setup occurring with vehicles traveling within the field of view. The system must be able to function day or night. Most systems overcome this problem by using separate detection algorithms for day and night operation. The system must be able to operate in variable environmental conditions such as fog, rain, and snow. While many algorithms adapt to these scenarios, performance is degraded by such conditions. The system mounting must be extremely stable since camera motion can adversely affect detection capabilities. As with most overhead sensors, two closely spaced vehicles may be difficult to separate. However, this may not be significant if the goal of the system is to provide overall traffic measurement parameters.

\subsubsection{Advantages and Disadvantages}

Advantages of a video processing system are in the wealth of information that is obtainable from an imaging system. Future advances in image processing techniques may yield more and more traffic information from the entire image and from changes in the successive images. The camera has the potential of being a true wide-area sensor in a limited sense, although it is not being utilized to its fullest extent at the present time because of processing limitations. However, these systems can perform as the functional equivalent of many inductive detectors within the field of view. For areas with complex sensing requirements, these systems may be much more cost-effective than traditional sensing methods.

Besides the installation advantages of other overhead sensors, the video image processing system often has user configurable and reconfigurable zones of interest for monitoring. This gives the system the flexibility to be quickly adaptive to temporary or permanent adjustments to vehicle thoroughfares. For example, if a lane is to be closed for repair work, the zones to monitor can be adjusted to assist in optimizing traffic flow during the repair work.

Disadvantages include degraded performance under adverse weather conditions. Many systems experience their poorest accuracy performance in the dusk or dawn hours as they undergo a transition from their daytime to nighttime detection algorithms. Unfortunately, this often corresponds with the time of peak traffic demands.

It is indisputable that video image processing is a leading candidate technology for widearea surveillance. In fact, many times the term "wide-area detection" is assumed to imply video imaging technology. One of the main appeals of video image processing for surveillance is its analogy to human vision. Although video images carry a wealth of information, there will remain a need for other sensors to efficiently provide much of the data needed to develop a description of traffic flow. 


\subsubsection{Present Research Efforts}

A tremendous amount of research is ongoing regarding video image processing for IVHS applications. Several major research programs have centered around video image processing techniques for "wide-area detection." Among these are the University of Minnesota's Autoscope system (Michalopoulos 1991), the TRIP II system (Dickinson and Wan 1989), and the TITAN system (Blosseville et al. 1989).

Some of the earliest research involving traffic parameter measurement was performed by the Jet Propulsion Laboratory in the late 1970s. At that time computational capabilities were not available to perform the image processing algorithms in real time in a cost-effective way. Even at this early date, however, the potential of wide-area detections to measure new parameters from which new incident detection and signal control algorithms could be developed was recognized (Schlutsmeyer 1982).

Autoscope is probably one of the best developed and most extensively deployed video image processing systems in the United States at this time. Autoscope has been deployed as the primary surveillance tool to monitor 90 of 95 intersections for an integrated test of an Advanced Traffic Management System (ATMS) using the Sydney Coordinated Adaptive Traffic System (SCATS), one of the premier adaptive control systems developed to date. Autoscope uses detection regions selected by using interactive computer graphics to monitor regions and produce information about traffic in those regions. A single camera can thus emulate many loops. For several tests, Autoscope has demonstrated a greater than $95 \%$ detection accuracy under a variety of environmental conditions and during the day-to-night transition time. The presence detection algorithm was modified to meet SCATS detector requirements, and for certain configurations side-by-side detector zones are required to prevent missed detections or to minimize false detections (Michalopoulos et al. 1994).

The TRIP II system developed in England relies on processing of the entire video image. This system development path is concentrating on applying neural network pattern recognition techniques to traffic monitoring (Dickinson and Wan 1989).

The French research group INRETS in conjunction with CMM/EMP in France developed the TITAN video image processing system. This system is designed around the concept of performing automatic lane detection, then vehicle detection, and then building trajectories of detected vehicles from which traffic measurements are extracted. Processing speeds have proven to be something of a limitation for this concept (Blosseville et al. 1989).

Enkelmann discussed two methods for evaluation of optical flow fields from image sequences. This is an alternative video technique for detecting and tracking moving objects and detecting stationary objects. These techniques may benefit from work in simulator flow field properties (Enkelmann 1989).

Incident detection can be included as a subset of the capabilities of a traffic sensing video image processing system. This involves a higher level of information understanding than mere transmission of traffic parameters. Several traffic parameters are generally related in some way and are compared to thresholds for the identification and classification of an incident.

Autoscope has the capability of performing incident detection by placing two or more speed traps along each lane of traffic at the widest possible spacing within the field of view. These data are tracked and compared to historical data, and an alarm occurs when a threshold is exceeded. A second technique is under development which relies on image processing and image understanding capabilities (Michalopoulos 1991).

Typical video processing systems utilize two basic approaches. In the most popular approach, the system attempts to emulate existing point detectors using small subregions of 
the image; this approach limits incident detection algorithms to conventional techniques. Another approach is to detect and track individual vehicles within the scene; this is very expensive computationally. A different technique that attempts to periodically analyze the pattern of the traffic movement without tracking individual vehicles is being developed and tested in Scotland. IMPACTS is an incident detection system that attempts to mimic the human's ability to identify the state of congestion based on a qualitative description of moving and stationary traffic in a scene. For this technique, the entire roadway is subdivided into areas called cells of varying size depending on the perspective in the image and the anticipated nature of traffic movement. Cells are analyzed to determine the state of traffic movement within the cells, and cells are analyzed with respect to each other to detect platoons, queues, etc. Descriptions are compared over five cycles to analyze changes with respect to time, which enhances system reliability. This system was installed in Glasglow for incident detection in the summer of 1993. Quantitative results are not yet available, but first impressions indicate that the system is functioning correctly and appears stable under a variety of conditions (Hoose 1989; Dalgleish and Hoose 1994).

The TITAN image processing sensor was developed to perform the tasks of detecting incidents, classifying them, and calculating a confidence measure of the assessment. These detections are based on traffic measures or on direct detection (i.e., stationary vehicles on the shoulder, abnormal vehicle behavior, very fast or very slow moving vehicles) (Blosseville 1991).

\subsection{OTHER SENSING TECHNIQUES AND SPECIAL SUBSETS}

\subsubsection{AVI Technology}

Automatic Vehicle Identification is a technique for identifying and tracking vehicles which is emerging primarily in the commercial trucking industry. An electronic transponder (tag) with a unique identifier is placed on a vehicle, and devices for detecting the passage of a tagged vehicle are located along the roadway. When a vehicle with a tag passes a detector, the identity of the vehicle, its location, the time of its passage, and possibly other data are transmitted to a central data collection computer system. Primary applications of this technology to date have been for automatic toll collection and as a tracking tool for commercial vehicles for the purposes of safety, improved productivity, and improved audit tracking. Because other systems make use of this technology, this information could be a "free" means of collecting additional traffic data. The data collected could be volume and headway-type data, or, more importantly, the information from vehicles passing two stations a known distance apart can be combined in order to calculate travel time or average velocity. With the exception of an event occurring between two stations, calculation of the travel time or average velocity has generally been the more effective measure for detecting traffic congestion. The effectiveness of this technique for congestion monitoring depends on the number of tagged vehicles representing a significant proportion of the traffic. This technique is much more effective at times of heavy usage and much weaker during times of traditional low utilization (Hallenbeck, Boyle, and Ring 1992).

AVI tags can be purchased for $\$ 10-\$ 20$ in bulk quantities, but in small quantities, they run $\$ 50-\$ 100$ per tag. Issues in the uniform application of AVI technology for counting include (1) Who pays for purchase of the tag? (2) Who has access to the traveler's data and how are they used? (3) Can such a tag be made mandatory and what privacy rights are 
violated? Although widespread use of AVI probably should not be assumed for the purpose of examining AVI as a primary traffic surveillance tool, it is likely that for many scenarios AVI information could be used to help provide a measure of traffic flow and as a supplement to traffic detection and description. Present AVI systems may use microwave, radio frequency, or optical systems for identification (Hallenbeck, Boyle, and Ring 1992). A uniform AVI system is highly preferable to ensure consistent operation throughout a highway system (Grenzeback, Stowers, and Boghani 1988).

As a wide-area surveillance technology, AVI systems offer measures of actual travel times through a section of roadway, improved information for route guidance functions, and an additional means of incident or congestion detection. Because of the costs involved in developing, installing, and maintaining such an AVI system, this technology is best suited for wide-area surveillance use when required for some other IVHS function such as toll collection or commercial vehicle tracking.

Data from the operational Heavy Vehicle Electronic License Plate (HELP) tests indicate that $A V I$ readers are capable of detecting vehicles at speeds of up to $110 \mathrm{mph}$ and can detect tagged vehicles passing simultaneously in adjacent lanes. The AVI equipment worked in heavy rain and in simulated snow and ice. The system also seemed to function well in the presence of cellular phones, CB radios, and other forms of electromagnetic interference (Hallenbeck, Boyle, and Ring 1992).

All vehicles on the road need not be AVI tagged to effectively use this information. If an adequate sampling of vehicles carry such tagging, very good flow information is available. In fact, having all vehicles tagged probably would add little information and would greatly increase the processing burden for determining flow. However, it is not presently known what percentage of vehicles should be tagged in order to assume that they constitute a representative sample of the entire traffic pattern.

\subsubsection{Probe Vehicles}

The technology necessary to implement a probe vehicle scheme is essentially very similar or identical to the AVI technology. The difference in this concept lies in how it is applied. Specific vehicles act as probes, being driven through the highway network at controllable times and through a known path past several detectors to get travel time measures with the possibility of additional traffic flow insight from a human driver. This technique allows the measure of some parameters that would be impossible or very difficult without extensive tracking of individual vehicles. These vehicles can gather important input regarding where traffic delays occur and can quantify travel times across several control nodes for a better integrated view of overall traffic flow over a multiple-mile area. This type of program could be handled in several ways. Individual citizen volunteers who regularly pass through a wide area could have their vehicles equipped with tagging equipment. Another implementation could involve paid drivers in a tagged vehicle who follow a prescribed route while remaining with the flow of traffic. In addition to information provided through instrumentation, an implementation could also utilize paid drivers who are trained to evaluate and describe the traffic flow in much the same way as today's radio traffic reporters.

Both AVI and probe vehicles are also good candidates for providing a measure of the effectiveness of the TFWAS system. In addition, certain parameters such as net travel time and measurement of delay can be extracted from these techniques. 


\subsubsection{Synthetic-Aperture Radar}

Synthetic-aperture radar (SAR) is based on the creation of an effective long or large antenna by signal processing means rather than by the actual use of a long or large physical antenna. Often, only a single, relatively small, physical antenna is used, as is the case aboard an aircraft. (In the case of an airborne ground-mapping radar system, the antenna usually is mounted to be side-looking, and the motion of the aircraft carries the radiating element to each of the positions of the array. These array positions are the location of the physical antenna at the times of transmission and reception of the radar signals.) Typically, when one considers a synthetic aperture, one makes reference to the characteristics of a long linear array of physical antennas. In the case of an actual linear array, a number of radiating elements are assembled and placed at appropriate points along the array elements. Similarly, when the array is used as a receiver, the elements receive signals simultaneously. In both the transmitting and the receiving modes, waveguide or other transmission line interconnections are used, and interference phenomena are exploited to get an effective radiation pattern.

In the synthetic antenna case, however, only a single, relatively small radiating element is used in most instances. The antenna itself is translated to take up positions along a line. At each of these positions a signal is transmitted, and the radar signals received in response to that transmission are stored for subsequent data processing. It is essential that the storage be such that both amplitude and phase of the received signals are preserved.

After the radiating element has traversed some effective distance, the signals in storage strongly resemble the signals that would have been received by the elements of an actual array. Consequently, if the stored signals are subjected to the same operations as those used in forming a physical linear array, one can get the effect of a long antenna aperture. The Doppler principle and special data processing techniques are typically employed to synthesize the azimuth resolution of a very narrow beam, the same as that produced by a very large aperture antenna, resulting in very high resolution radar images. This idea has resulted in the use of the term "synthetic aperture" to designate this technique (Skolnik 1990).

Because the primary advantage of SAR is a moving reference frame, or "translating" antenna, SAR itself would probably have only limited application to highway wide-area surveillance where the reference point is most likely stationary. An actual linear array of physical antennas, as described above, would probably be more practical. Furthermore, because of the extensive signal processing requirements imposed by SAR, conventional radar systems would undoubtedly be more cost-effective for highway applications, at least in the near term.

\subsubsection{3-D Laser Imaging}

Laser cameras form a range image by scanning a scene with a focused amplitude modulated infrared laser beam. The laser beam is swept across and up and down the field of view by rotating mirrors. The reflected light from the scene is detected by a receiver that measures the intensity and phase of the return light. The range distance to each point in the image is calculated from the phase shift caused by the time delay of the returned light.

At present, most 3-D laser imaging systems are very expensive, ranging from $\$ 50,000$ to $\$ 100,000$. These systems are primarily used for laboratory or military applications. It will be costly to harden such devices for continuous environmental battering. There are also safety concerns regarding the scanning laser. 
A suite of 3-D laser imaging sensors is being used to monitor vehicle movements in a joint study by the National Highway Safety Administration, the University of Michigan's Transportation Research Institute, and the Environmental Research Institute of Michigan (IVHS 1994).

The sensors will be mounted above the roadway and will detect vehicles as changes in elevation from the roadway. The system will measure the position and movement of vehicles as a function of time. The purpose of the system is to develop a database of vehicle interactions to determine what actions may indicate an impending crash and what interactions will be harmless. This information will be useful in developing anticipatory crash avoidance technologies.

\subsubsection{Weigh-in-motion Detectors}

Weigh-in-motion (WIM) sensors measure the instantaneous weights of the axles of traveling vehicles. Modern WIM sensors are usually made of two piezoelectric cables mounted in a slot in the road used in conjunction with an inductive loop between them. The weight of an axle can be calculated by integrating the output of the sensors over time and multiplying by the speed of the vehicle and a calibration factor. The speed is calculated by computing the time for the axle to travel between the two cables. The inductive loop is used to detect the end of the vehicle so that the sum of the axle weights can be calculated. Reliability and accuracy of these WIM systems for predetection of illegally loaded vehicles has not been good traditionally (Newton, Frith, and Barbour 1992). Piezoelectric vehicle detectors do not operate very efficiently at very low speeds. Tests using this equipment in the United Kingdom revealed that accuracy tended to be low when compared to static weight measures. Additionally, systems required recalibration, probably because of temperature changes (Sullivan, Lloyd, and Bonnet 1992).

The use of fiber-optic technology has shown some preliminary promise as a technique for weighing vehicles. A fiber-optic cable illuminated at one end is stretched across the path of the vehicle. A detector on the opposite end of the fiber measures the strength or phase of the light emerging from the cable. As a vehicle passes over the cable, the cable is compressed, and the light emerging from the fiber decreases. The heavier the vehicle, the greater the reduction in light intensity. This technique could possibly be used to detect vehicle passage/presence at lower speeds. A crude classification of traffic is also possible according to weight. This technology is not particularly well suited to wide-area surveillance at this time. A major drawback of this technology presently is the poor life expectancy due to repeated compressions of the cable (Stevens and Spindlow 1990). 


\section{EVALUATION OF TECHNOLOGY CAPABILITIES FOR TFWAS SYSTEM REQUIREMENTS}

In the attempt to evaluate the capabilities of the relevant sensor technologies to meet the desired system requirements for TFWAS, each of the 12 technologies was assessed relative to a series of parameters identified as representing the most pertinent system descriptors. These parameters, along with each technology class, are presented in Tables 5.1 to 5.4. Although each table is designed to stand alone, the function and philosophy behind each is described in this section.

\subsection{OVERVIEW OF TECHNOLOGIES}

Table 5.1 addresses the function, operation, and utility for each technology, followed by an intuitive estimate of its probable system cost and implementation timeline. The "function" of each technology attempts to describe the purpose this technology is intended to serve, that is, why it is being used in the first place. For example, one can use acoustic technology to measure the presence of vehicles, determine their passage with which a vehicle count can possibly be obtained, calculate their speed, and to a great extent classify the vehicles. Probe vehicles, on the other hand, would be driven by a person who would function much like a present-day traffic reporter.

The description under the heading of "operation" attempts to summarize the physical principle upon which each technology depends. For example, a magnetometer measures the perturbation of the local earth magnetic field induced when a ferromagnetic body like an automobile passes over it.

"Utility" addresses the strengths and weaknesses of each technology. Again as an example, active infrared is a very precise technology capable of very accurately performing several functions and is applicable day or night. However, because it involves a laser, there is a concern for visual safety. Furthermore, it is vulnerable to adverse atmospheric conditions, such as smog, fog, mist, or rain.

The cost and timeline columns were added to Table 5.1 to give the user a feel for the expected investment required for each technology, as well as some insight as to when the sensors could be on-line in the field. Although the "timeline" parameters of short term, near term, and future are defined in a table footnote, an expansion of these definitions is warranted here. "Short term" implies that sensors for this technology are readily available commercially, and most of this type have already been in service for traffic applications. "Near term" implies that some sensors are commercially available and that there may even be some limited experience with their application for traffic. Either way, very little development or modification would be required to bring them on line for a traffic function. "Future" technology is something that is not immediately available for general highway applications. These sensors are either in the development/prototype stage or are quite possibly in other general use, such as for aerospace or Department of Defense applications, but are presently expensive and their conversion for traffic use would involve considerable cost. In other words, some investment will be required to make this technology cost-effective for highway applications. The cost reference in Table 5.1 refers to the total system cost as opposed to the individual sensor and should be used in conjunction with Table 5.2. 
Table 5.1. System considerations for TFWAS system technology

\begin{tabular}{|c|c|c|c|c|c|}
\hline Technology & Function & Operation & Utility & $\operatorname{Cost}^{b}$ & Timeline $^{c}$ \\
\hline Inductive loop & $\begin{array}{l}\text { Detect small area presence; } \\
\text { measure volume, speed }\end{array}$ & $\begin{array}{l}\text { Excited by signals from } \\
10 \text { to } 200 \mathrm{kHz}\end{array}$ & $\begin{array}{l}\text { Simple; reliable when properly } \\
\text { installed; buried; limited } \\
\text { capability; susceptible to } \\
\text { damage from freezing weather }\end{array}$ & Low & Short term \\
\hline Magnetometer & $\begin{array}{l}\text { Detect small area presence; } \\
\text { replacement for inductive } \\
\text { loops }\end{array}$ & $\begin{array}{l}\text { Measures perturbation } \\
\text { of local earth magnetic } \\
\text { field }\end{array}$ & Simple; limited capability & $\begin{array}{l}\text { Low } \\
\text { to } \\
\text { med. }\end{array}$ & Short term \\
\hline $\begin{array}{l}\text { Weigh-in- } \\
\text { motion }\end{array}$ & $\begin{array}{l}\text { Measure weight of vehicle; } \\
\text { detect presence }\end{array}$ & $\begin{array}{l}\text { Measures changes in } \\
\text { capacitance of the sensor } \\
\text { element }\end{array}$ & $\begin{array}{l}\text { Trade-off of vehicle speed as a } \\
\text { function of accuracy; long-term } \\
\text { reliability unproven }\end{array}$ & Med. & Future \\
\hline Ultrasonics & $\begin{array}{l}\text { Measure volume, speed, } \\
\text { occupancy, presence, queue } \\
\text { length }\end{array}$ & $\begin{array}{l}\text { Emit frequencies greater } \\
\text { than } 20 \mathrm{kHz}\end{array}$ & $\begin{array}{l}\text { Applicable to nearly all } \\
\text { targets; relatively short range; } \\
\text { subject to attenuation from } \\
\text { environment, porous surfaces }\end{array}$ & $\begin{array}{l}\text { Low } \\
\text { to } \\
\text { med. }\end{array}$ & Near term \\
\hline $\begin{array}{l}\text { Microwaves } \\
\text { (including } \\
\text { millimeter } \\
\text { wave, } 1 \text { to } 100 \\
\mathrm{GHz} \text { ) }\end{array}$ & $\begin{array}{l}\text { Measure speed, volume, } \\
\text { presence, classification (via } \\
\text { profile), count }\end{array}$ & $\begin{array}{l}\text { Transmits near } 10.5 \mathrm{Ghz} \text {, } \\
24.0 \mathrm{Ghz} \text {, and } 34.0 \mathrm{Ghz} \text {, } \\
\text { the FCC-allocated } \\
\text { frequency bands }\end{array}$ & $\begin{array}{l}\text { Insensitive to weather; reliable; } \\
\text { provide day/night operations; } \\
\text { may lock on strongest } \\
\text { backscatter at exclusion of } \\
\text { small vehicles }\end{array}$ & $\begin{array}{l}\text { Low } \\
\text { to } \\
\text { med. }\end{array}$ & $\begin{array}{l}\text { Short to near } \\
\text { term }\end{array}$ \\
\hline Active infrared & $\begin{array}{l}\text { Laser radar; measures } \\
\text { presence, speed, volume, } \\
\text { occupancy, classification }\end{array}$ & $\begin{array}{l}\text { Transmits pulse in } \\
\text { near/mid infrared; } \\
\text { detects reflection }\end{array}$ & $\begin{array}{l}\text { Precise; applicable day or } \\
\text { night; visual safety concerns; } \\
\text { vulnerable to environment } \\
\text { (fog, mist, rain, etc.) }\end{array}$ & Med. & Near term \\
\hline
\end{tabular}


Table 5.1 (continued)

\begin{tabular}{|c|c|c|c|c|c|}
\hline Technology & Function ${ }^{a}$ & Operation & Utility & $\operatorname{Cost}^{b}$ & Timeline $^{c}$ \\
\hline $\begin{array}{l}\text { Passive } \\
\text { infrared }\end{array}$ & $\begin{array}{l}\text { Measures volume, presence, } \\
\text { occupancy, passage }\end{array}$ & $\begin{array}{l}\text { Detects emitted energy } \\
\text { difference between } \\
\text { background and target }\end{array}$ & $\begin{array}{l}\text { Simple; reliable; relatively easy } \\
\text { to implement; potentially } \\
\text { affected by fog and } \\
\text { precipitation }\end{array}$ & Low & Short term \\
\hline Acoustics & $\begin{array}{l}\text { Measures presence, speed, } \\
\text { passage, classification, } \\
\text { possibly count }\end{array}$ & $\begin{array}{l}\text { Microphone array } \\
\text { monitoring; range } 20 \text { to } \\
30 \mathrm{ft} \text { with } 20^{\circ} \text { beam }\end{array}$ & $\begin{array}{l}\text { Simple; easy to } \\
\text { install/maintain; reliable; } \\
\text { relatively immune to } \\
\text { environment; vulnerable to } \\
\text { unpredicted noise }\end{array}$ & $\begin{array}{l}\text { Low } \\
\text { to } \\
\text { med. }\end{array}$ & $\begin{array}{l}\text { Short to near } \\
\text { term }\end{array}$ \\
\hline $\begin{array}{l}\text { Video imaging } \\
\text { (includes the } \\
\text { near infrared) }\end{array}$ & $\begin{array}{l}\text { Area imaging; can estimate } \\
\text { speed, volume, presence, } \\
\text { occupancy, count, passage, } \\
\text { queue length, incidents, and } \\
\text { pending incidents }\end{array}$ & $\begin{array}{l}\text { Detects and images from } \\
\text { visible to near/mid } \\
\text { infrared }\end{array}$ & $\begin{array}{l}\text { Can assess state of a region; } \\
\text { algorithms available for } \\
\text { highway; potentially identify } \\
\text { pending incidents; vulnerable } \\
\text { to environment (fog, mist, rain, } \\
\text { etc.) }\end{array}$ & $\begin{array}{l}\text { Low } \\
\text { to } \\
\text { med. }\end{array}$ & $\begin{array}{l}\text { Short to near } \\
\text { term }\end{array}$ \\
\hline $\begin{array}{l}\text { 3-D laser } \\
\text { imaging } \\
\text { systems }\end{array}$ & $\begin{array}{l}\text { Measure speed, volume, } \\
\text { count, passage, presence, } \\
\text { classification, pending } \\
\text { incidents }\end{array}$ & $\begin{array}{l}\text { Like active infrared but } \\
\text { is able to discriminate } \\
\text { elevation }\end{array}$ & $\begin{array}{l}\text { Similar to active infrared; used } \\
\text { to develop vehicle interaction } \\
\text { database to determine pending } \\
\text { crash from harmless } \\
\text { interactions }\end{array}$ & High & Future \\
\hline $\begin{array}{l}\text { Synthetic } \\
\text { aperture radar }\end{array}$ & $\begin{array}{l}\text { Measure speed, volume, } \\
\text { count, presence, classification } \\
\text { (via profile) }\end{array}$ & $\begin{array}{l}\text { Computationally } \\
\text { intensive; moving } \\
\text { reference frame }\end{array}$ & $\begin{array}{l}\text { Insensitive to weather; day/ } \\
\text { night operations; very limited } \\
\text { applicability with stationary } \\
\text { reference frame }\end{array}$ & High & Future \\
\hline
\end{tabular}


Table 5.1 (continued)

\begin{tabular}{|c|c|c|c|c|c|}
\hline Technology & Function ${ }^{a}$ & Operation & Utility & $\cos t^{b}$ & Timeline $^{c}$ \\
\hline $\begin{array}{l}\text { AVI } \\
\text { technologies }\end{array}$ & $\begin{array}{l}\text { Identify/track vehicles; } \\
\text { measure speed, volume, } \\
\text { count, classification }\end{array}$ & $\begin{array}{l}\text { Tagged vehicles pass } \\
\text { reader for automatic } \\
\text { identification }\end{array}$ & $\begin{array}{l}\text { Simple; reliable; measures } \\
\text { actual travel time }\end{array}$ & Med. & Near term \\
\hline Probe vehicles & $\begin{array}{l}\text { Direct human involvement to } \\
\text { obtain travel time and } \\
\text { implicit parameters such as } \\
\text { traffic flow insight, delays, } \\
\text { incidents }\end{array}$ & $\begin{array}{l}\text { Vehicle driven through } \\
\text { highway network known } \\
\text { path at controlled times }\end{array}$ & $\begin{array}{l}\text { Allows measurement of some } \\
\text { parameters that would be very } \\
\text { difficult without extensive } \\
\text { individual tracking; permits } \\
\text { traffic flow insight by humans }\end{array}$ & Low & Short term \\
\hline
\end{tabular}

aCost refers to the system cost, as contrasted with the cost of the individual sensor.

'Function: Any single sensor may not be capable of performing all functions; however, two or more sensors acting together can accomplish any function identified.

'Timeline: Short term-<1 year; commercially available; near term-1 to 5 years; some commercial availability; future $\rightarrow>5$ years; development/prototype stage, not immediately available for highway applications. 
Table 5.2 Estimated cost considerations for TFWAS system technology

\begin{tabular}{|c|c|c|c|}
\hline Technology & Capital & Installation ${ }^{Q}$ & Maintenance ${ }^{b}$ \\
\hline Inductive loop & $\$ 10 \mathrm{~K}$ & Low & Low to medium \\
\hline Magnetometer & \$10K & Low & Low to medium \\
\hline Weigh-in-motion & $\$ 100 \mathrm{~K}$ & High & High \\
\hline Ultrasonics & $\$ 10 \mathrm{~K}$ & Low to medium & Low to medium \\
\hline Microwaves & $\$ 40 K$ & Low to medium & Medium \\
\hline Active infrared & $\$ 250 \mathrm{~K}$ & High & High \\
\hline Passive infrared & $\$ 60 \mathrm{~K}$ & Low & Low \\
\hline Acoustics & $\$ 10 K$ & Low to medium & Low to medium \\
\hline Video imaging & $\$ 10 \mathrm{~K}$ & Low & Low to medium \\
\hline $\begin{array}{l}\text { 3-D laser imaging } \\
\text { systems }\end{array}$ & $\$ 500 \mathrm{~K}$ & High & High \\
\hline $\begin{array}{l}\text { Synthetic-aperture } \\
\text { radar }\end{array}$ & $\$ 300 \mathrm{~K}$ & High & High \\
\hline AVI technologies & $\$ 100 \mathrm{~K}$ & Medium & Medium to high \\
\hline Probe vehicles & \$30K & Low & High $^{c}$ \\
\hline
\end{tabular}

Installation: Low-<\$5 per sensor; medium $-\$ 5 \mathrm{~K}$ to $\$ 15 \mathrm{~K}$ per sensor; high $\rightarrow$ S15K per sensor.

bMaintenance: Low-<\$1K per sensor per year; medium $-\$ 1 K$ to $\$ 10 \mathrm{~K}$ per sensor per year; high $\rightarrow \$ 10 \mathrm{~K}$ per sensor per year. Maintenance includes related recurring costs but does not include cost of operations.

'Includes driver's salary. 
Table 5.3. Statistical and systems measures for TFWAS system tochnology

\begin{tabular}{|c|c|c|c|c|c|c|}
\hline \multirow[b]{2}{*}{ Technology } & \multicolumn{6}{|c|}{ Statistical and systems measures } \\
\hline & $\begin{array}{l}\text { Classification of } \\
\text { vehicles }\end{array}$ & $\begin{array}{l}\text { Capability of } \\
\text { determining } \\
\text { vehicle } \\
\text { separationa }\end{array}$ & $\begin{array}{l}\text { Determination of } \\
\text { approach queue } \\
\text { length }^{b}\end{array}$ & $\begin{array}{l}\text { Determination } \\
\text { of time in } \\
\text { queue }^{e}\end{array}$ & $\begin{array}{l}\text { Determination of } \\
\text { vehicle miles } \\
\text { traveled }^{d}\end{array}$ & Predictive capabilities \\
\hline Inductive loop & $\begin{array}{l}\text { Limited to variations } \\
\text { in vehicle length }\end{array}$ & Limited & Limited & Limited & High & $\begin{array}{l}\text { If located in turn } \\
\text { lanes and/or on-off } \\
\text { ramps }\end{array}$ \\
\hline Magnetometer & $\begin{array}{l}\text { Limited to variations } \\
\text { in vehicle length }\end{array}$ & Limited & Limited & Limited & High & $\begin{array}{l}\text { If located in turn } \\
\text { lanes and/or on-off } \\
\text { ramps }\end{array}$ \\
\hline Weigh-in-motion & $\begin{array}{l}\text { Can classify via weight } \\
\text { but limited to } \\
\text { individual variations }\end{array}$ & Limited & Limited & Limited & High & $\begin{array}{l}\text { If located in turn } \\
\text { lanes and/or on-off } \\
\text { ramps }\end{array}$ \\
\hline Ultrasonics & $\begin{array}{l}\text { Limited to variations } \\
\text { in vehicle length }\end{array}$ & Limited & Limited & Limited & High & $\begin{array}{l}\text { If located in turn } \\
\text { lanes and/or on-off } \\
\text { ramps }\end{array}$ \\
\hline $\begin{array}{l}\text { Microwaves } \\
\text { (including } \\
\text { millimeter wave, } \\
1 \text { to } 100 \mathrm{GHz} \text { ) }\end{array}$ & Can classify via profile & Moderate & $\begin{array}{l}\text { Moderate to } \\
\text { extensive }\end{array}$ & Moderate & Moderate & $\begin{array}{l}\text { Can see limited } \\
\text { behavior trends; can } \\
\text { be located at turn } \\
\text { lanes and ramps }\end{array}$ \\
\hline Active infrared & $\begin{array}{l}\text { Can classify essentially } \\
\text { all vehicles (single or } \\
\text { groups) }\end{array}$ & Moderate & Moderate & Moderate & Limited & $\begin{array}{l}\text { Imaging ir sensors } \\
\text { can see traffic } \\
\text { behavior and judge } \\
\text { trends }\end{array}$ \\
\hline
\end{tabular}


Table 5.3 (continued)

\begin{tabular}{|c|c|c|c|c|c|c|}
\hline \multirow[b]{2}{*}{ Technology } & \multicolumn{6}{|c|}{ Statistical and systems measures } \\
\hline & $\begin{array}{c}\text { Classification of } \\
\text { vehicles }\end{array}$ & $\begin{array}{c}\text { Capability of } \\
\text { determining } \\
\text { vehicle } \\
\text { separation }\end{array}$ & $\begin{array}{l}\text { Determination of } \\
\text { approach queue } \\
\text { length }^{b}\end{array}$ & $\begin{array}{l}\text { Determination } \\
\text { of time in } \\
\text { queue }^{c}\end{array}$ & $\begin{array}{c}\text { Determination of } \\
\text { vehicle miles } \\
\text { traveled }^{d}\end{array}$ & Predictive capabilities \\
\hline Passive infrared & Limited & Limited & Limited & Limited & Moderate & Very limited \\
\hline Acoustics & $\begin{array}{l}\text { Can classify via sound } \\
\text { patterns }\end{array}$ & Limited & Moderate & Moderate & Moderate & $\begin{array}{l}\text { If located in turn } \\
\text { lanes and/or on-off } \\
\text { ramps }\end{array}$ \\
\hline Video imaging & $\begin{array}{l}\text { Can classify essentialty } \\
\text { all vehicles (single or } \\
\text { groups) }\end{array}$ & Extensive & $\begin{array}{l}\text { Moderate to } \\
\text { extensive }\end{array}$ & Extensive & Limited & $\begin{array}{l}\text { Can see traffic } \\
\text { behavior and judge } \\
\text { trends }\end{array}$ \\
\hline $\begin{array}{l}\text { 3-D laser imaging } \\
\text { systems }\end{array}$ & $\begin{array}{l}\text { Can classify essentially } \\
\text { all vehicles (single or } \\
\text { groups) }\end{array}$ & Extensive & $\begin{array}{l}\text { Moderate to } \\
\text { extensive }\end{array}$ & Extensive & Limited & $\begin{array}{l}\text { Can see traffic } \\
\text { behavior and judge } \\
\text { trends }\end{array}$ \\
\hline $\begin{array}{l}\text { Synthetic- } \\
\text { aperture radar }\end{array}$ & Can classify via profile & Moderate & Moderate & Moderate & Moderate & $\begin{array}{l}\text { Can see limited } \\
\text { behavior trends; can } \\
\text { be located at turn } \\
\text { lanes and ramps }\end{array}$ \\
\hline AVI technologies & $\begin{array}{l}\text { Can classify via } \\
\text { pretagging of vehicle }\end{array}$ & Limited & Limited & Limited & High & $\begin{array}{l}\text { If scanners are } \\
\text { located at points of } \\
\text { probable direction } \\
\text { change }\end{array}$ \\
\hline
\end{tabular}


Table 5.3 (continued)

\begin{tabular}{|c|c|c|c|c|c|c|}
\hline \multirow[b]{2}{*}{ Technology } & \multicolumn{6}{|c|}{ Statistical and systems measures } \\
\hline & $\begin{array}{l}\text { Classification of } \\
\text { vehicles }\end{array}$ & $\begin{array}{l}\text { Capability of } \\
\text { determining } \\
\text { vehicle } \\
\text { separation }\end{array}$ & $\begin{array}{l}\text { Determination of } \\
\text { approach queue } \\
\text { length }^{b}\end{array}$ & $\begin{array}{l}\text { Determination } \\
\text { of time in } \\
\text { queue }^{c}\end{array}$ & $\begin{array}{l}\text { Determination of } \\
\text { vehicle miles } \\
\text { traveled }^{d}\end{array}$ & Predictive capabilities \\
\hline $\begin{array}{l}\text { Probe vehicles } \\
\text { (assumes a driver } \\
\text { on board) }\end{array}$ & $\begin{array}{l}\text { Driver can identify } \\
\text { vehicles }\end{array}$ & Extensive & Extensive & Extensive & Moderate & $\begin{array}{l}\text { Can see traffic } \\
\text { behavior and judge } \\
\text { trends }\end{array}$ \\
\hline
\end{tabular}

"Vehicle Separation:

Limited-Discrimination of individual vehicles through light traffic; may require two or more sensors.

Moderate-Can discriminate through light traffic to relatively steady flow.

Extensive-Can discriminate through steady flow to heavy traffic with multiple lane changes.

Approach Queue Length:

Limited-Very localized, such as on/off ramps; may require two or more sensors.

Moderate-Can discern maximum queue length as long as queue is within the field of view of the sensor.

Extensive-Can discern maximum queue length over a very large range (typically 3 to 5 miles).

'Determination of Time in Queue is defined as the ability of the sensor to estimate the average time a vehicle may spend in queue when the vehicle is within the field of view of an individual sensor.

Determination of Time in Queue:

Limited-Limited to passage of a single vehicle.

Moderate-In field of view for a few seconds.

Extensive-In field of view for several minutes.

${ }^{d}$ Vehicle Miles Traveled is traditionally defined as the product of the total number of vehicles using the roadway during a given time and the average trip length of each of the vehicies. However, with the exception of AVI technologies and probe vehicles, average trip length cannot be directly determined with the dominant TFWAS system technologies. Therefore, for the purpose of this table, the assumption is made that an average trip length can be established with AVI technologies and applied directly to the other technologies to assess their respective effectiveness in the determination of vehicle miles traveled. In other words, given the knowledge of the average trip length, the table attempts to assess how effective a specific technology is in establishing vehicle miles traveled.

Vehicle Miles Traveled:

Limited-Can only provide a qualitative estimate of vehicle miles traveled with no direct quantified data output for later analysis.

Moderate-Can provide a limited quantification of vehicle miles traveled; capable of some data storage for later analysis.

High-Can essentially quantify and sum each individual vehicle passing.

The determination of these parameters is computationally expensive. 
Table 5.4. Degree to which aggregate trafiic parameters can be determined by TFWAS technology

\begin{tabular}{|c|c|c|c|c|c|}
\hline \multirow{2}{*}{ Technology } & \multicolumn{5}{|c|}{ Aggregate traffic parameters } \\
\hline & Coverage $^{\circ}$ & Velocity & Acceleration & Density & Extent \\
\hline Inductive loop & Limited & Moderate per lane & Derived & High per lane & Limited \\
\hline Magnetometer & Limited & Moderate per lane & Derived & High per lane & Limited \\
\hline Weigh-in-motion & Limited & Limited & Derived & High per lane & Limited \\
\hline Ultrasonics & Limited & High per lane & Derived & High per lane & Limited \\
\hline $\begin{array}{l}\text { Microwaves (including millimeter } \\
\text { wave) }\end{array}$ & Moderate & High & Derived & High & Moderate \\
\hline Active infrared & Extensive & Moderate & Derived & High per lane $e^{e}$ & Limited \\
\hline Passive infrared & Limited & Limited & Derived & Moderate & Limited \\
\hline Acoustics & Moderate & High & Derived & High per lane & Limited \\
\hline Video imaging & Extensive & Moderate & Derived & Highe $^{*}$ & Extensive \\
\hline 3-D laser imaging systems & Extensive & Moderate & Derived & High & Moderate \\
\hline Synthetic-aperture radar & Moderate & Highe & Derived & Highe $^{e}$ & Limited \\
\hline
\end{tabular}


Table 5.4 (continued)

\begin{tabular}{|c|c|c|c|c|c|}
\hline \multirow{2}{*}{ Technology } & \multicolumn{5}{|c|}{ Aggregate traffic parameters } \\
\hline & Coverage $^{a}$ & Velocityb & Acceleration & Density ${ }^{c}$ & Extent \\
\hline AVI technologies & Limited & Moderate & Derived & Highs $^{8}$ & Moderate \\
\hline Probe vehicles & Extensive & Moderate & Estimated & Limited & Extensive \\
\hline
\end{tabular}

${ }^{a}$ Coverage:

Limited-Very localized; may require two or more sensors.

Moderate-Can discern up to a half mile.

Extensive-Can discern from a half mile to several miles (typically 3 to 5 miles).

'Velocity:

Limited-Can only provide a qualitative estimate of aggregate traffic velocity with little or no direct quantified data output for

later analysis.

Moderate-Can provide a limited quantification of aggregate traffic velocity; capable of some direct quantified data output for

later analysis.

High-Can quantify velocity of virtually each individual vehicle passing, although may require a minimum of two sensors.

'Density:

Limited-Can only provide a qualitative estimate of aggregate traffic with no direct quantified data output for later analysis.

Moderate-Can provide a limited quantification of aggregate vehicle density; capable of some direct quantified data output for later analysis.

High-Can essentially quantify and sum each individual vehicle passing.

${ }^{d}$ Extent implies how well the specific technology can apply to and perform the TFWAS system function and how much aggregate status information can be derived by the observer.

Extent:

Limited-Only a limited amount of specific information is received; marginal or inadequate to determine overall traffic status.

Moderate-Able to integrate some events and present a broader scope of information to enable an observer to determine some trends.

Extensive-Technology contributes significantly to the TFWAS system function and presents sufficient information to allow an observer to adequately determine aggregate traffic status.

The determination of these parameters is computationally expensive.

tVery effective for detecting pedestrians and large animals (e.g., deer).

sAlthough limited as a direct measurement, AVI technologies can provide a good statistical estimate of density. 
A rough-order-of-magnitude cost estimate for each technology is presented in Table 5.2. The table has been divided into three cost phases: capital, installation, and maintenance. The "capital" cost is an estimate of what one would expect to pay for a sensor and its supporting infrastructure, that is, its hardware and software. "Installation" is the expected cost to put that capability in place and bring it on line. The discriminating parameters of low, medium, and high (defined in the footnotes) were selected where "low" implies that the installation cost per sensor for labor and equipment would generally be less than \$5K An inductive loop installed in a road, especially prior to surfacing, would be an example. A sensor installation that would require between $\$ 5 \mathrm{~K}$ and $\$ 15 \mathrm{~K}$ was assumed to be of "medium" cost. The AVI technologies that would require the installation of code readers periodically along the highway, the encoded strips on vehicles, and the inherent data processing equipment would likely amortize as a medium cost. "High" costs are defined as those installations that may require labor- and/or equipment-intensive infrastructure, such as special mounting configuration, environmental protection, or sophisticated data processing/computational equipment. These installations would likely exceed $\$ 15 \mathrm{~K}$ per sensor. The 3-D laser imaging systems would probably meet this criterion.

A similar range of discriminators was used for maintenance cost. "Low" maintenance cost implies that the sensor, once in place, is generally sufficiently reliable that very little additional attention is required. The maintenance costs for these would be expected to fall below \$1K per year per sensor. An inductive loop that may cost \$2K to replace but would need replacement only once in 3 years would be an example. "Medium" maintenance cost is for sensors that one would expect to require more attention, either in the form of labor or in replacement hardware. A likely cost range would be on the order of $\$ 1 \mathrm{~K}$ to $\$ 10 \mathrm{~K}$ per sensor per year. A "high" maintenance cost would be for those sensors that are comprised of moving parts subject to wearout and/or whose maintenance is labor intensive. A maintenance operation exceeding $\$ 10 \mathrm{~K}$ per sensor per year would be considered high. The optical fibers in the WIM sensors would probably have a limited cycle life and need frequent replacement, and the driver's salary for the probe vehicle would surely exceed $\$ 10 \mathrm{~K}$. Operations costs, however, are not included as part of maintenance.

\subsection{TECHNOLOGY CAPABILITIES FOR TFWAS}

Table 5.3 attempts to quantify the statistical and systems measures for the identified technologies. The statistical and systems measures are the criteria that define how well each technology can perform its function as a wide-area surveillance technology. The following

parameters were selected as the most important quantifiers of this capability: classification of vehicles, determination of vehicle separation, approach queue length, time in queue, vehicle miles traveled, and predictive capabilities. The ideal technology would be one that scores high in all categories.

The capability of classifying vehicles implies how well the specific technology can identify individual vehicles and classes of vehicles (i.e., truck vs passenger car, heavy vehicle vs light, long vs short, and possibly even specific make and model). For example, video imaging with a sufficiently sophisticated processing algorithm should be able to classify vehicles.

Vehicle separation capability is a measure of how well the sensor technology can discriminate individual vehicles. The three classifications of limited, moderate, and extensive have been defined for this category. A sensor is said to have limited vehicle separation capability if it is only capable of discriminating individual vehicles in conditions of light traffic 
and if it requires two or more sensors to accomplish this. A moderate capability is said to exist when the sensor can discriminate vehicle separation through light- to steady-flow traffic. A technology would have an extensive vehicle separation capability if it can discriminate individual vehicles through a steady to heavy traffic flow where multiple lane changes are occurring. Visible imaging with the correct algorithms, for example, could discern this.

Approach queue length was taken to mean how well the specific sensor technology can discern the formations and lengths of vehicle lines at entrances, exits, and other traffic-serving regions. The same three classifications of limited, moderate, and extensive were used here as were used for vehicle separation. Here, however, "limited" is defined as a very localized capability, such as on or off ramps, and multiple sensors are very likely required. The maximum discernible queue length would be limited to the most distant sensor with the implicit assumption that the queue is continuous between sensors. A "moderate" capability is said to exist when the sensor can discern a maximum queue length, as long as the queue itself is within the field of view of the sensor. The actual sensing range would, of course, vary as a function of the specific sensor, but one should expect discrimination on the order of a quarter to a half mile. "Extensive" capability is where the sensor can discern maximum queue length over a very large range, say up to 3 to 5 miles. A video imager could accomplish this on a clear day.

The determination of the time in queue is defined as the capability of the sensor technology to measure the rate of passage of a vehicle through the queue within the field of view of the sensor. Again, the same discriminating parameters of limited, moderate, and extensive are used, although here they are referencing time. "Limited" is the rate of passage of a single vehicle and is subject to inherent inaccuracies induced by such factors as the length of the vehicle or a variation of speed between sensors outside the field of view. "Moderate," meanwhile, implies that the vehicle will be within the field of view of the sensor for at least a few seconds, and its rate of passage can be more accurately determined. "Extensive" implies that the vehicle will be in the field of view for several minutes, giving the sensor plenty of time to quantify the time in queue. In general, the better the capability of the sensor to discern the approach queue length, the better will be its capability to determine the vehicle's time in queue.

Vehicle miles traveled is a parameter that is traditionally defined as the product of the total number of vehicles using the roadway during a given time and the average trip length of each of these vehicles (i.e., VMT $=v_{n} \times l_{t}$, where $v_{n}$ is number of vehicles and $l_{t}$ is trip length). Because the average trip length for each vehicle is implicit in the definition, vehicle miles traveled cannot be explicitly quantified by the majority of the identified dominant technologies. However, through data collected with AVI technologies and/or probe vehicles, one could obtain a rough approximation of a "typical" trip length to use in this equation. When combined with the total number of vehicles using the roadway, a parameter that can be readily determined, the factoring in of this approximation of trip length could at least establish a normalized trend of vehicle miles traveled. This was the assumption made for the development of the discrimination range for vehicle miles traveled in Table 5.3.

The discriminators for vehicle miles traveled are limited, moderate, and high. "Limited" implies that the sensor technology can only provide a cursory or qualitative estimate of this parameter that would result in no direct quantified data output for later analysis. Visual imaging, for example, in the absence of a computationally expensive algorithm, would have a very limited capability of summing and storing the total number of vehicles traversing its sensor during any given time. A "moderate" assessment implies that the sensor is capable of some quantification of vehicle miles traveled, in the sense that there is at least some limited 
vehicle passage data stored for later analysis. The vehicle count information gleaned from microwave sensors, for example, may be degraded by clutter, thus compromising an actual vehicle count. A "high" rating implies that not only is there automated permanent data storage, but the sensor can also determine the vehicle passage very accurately. Vehicles passing an inductive loop, for example, can be very accurately counted, and the count information can be very easily stored.

The predictive capability of a technology is its ability to anticipate future events based on the occurrences it is presently observing. The observation of a lane change to the left-turn bay would be a very strong indicator that a left turn was pending. Some of the sensor technologies by their very nature would necessarily have limited predicative capabilities unless the sensors themselves were intentionally located in positions where event changes are naturally expected, such as in turn lanes. However, even with this limited capability, much information of anticipated traffic flow can still be obtained. The placement of sensors at decision points where, when activated, they would suggest a very probable next event is one way to maximize predictive capabilities. An ideal predictive sensor would be one that can "see" traffic behavior and anticipate its trends, especially if the sensor could judge the onset of a pending incident or accident and take immediate actions to minimize its impact. Video imaging has a degree of this capability wherein, based on such observations as multiple lane changes and brake lights, it can judge trends. However, to date the most reliable prediction of pending incidents is still from observations made by the drivers of probe vehicles. One should note, however, that other statistical and systems parameters, such as vehicle classification and time in queue, also may provide useful predictive information. For example, the turning time for a large truck is longer than that for other vehicles, and the truck is probably more likely to follow a common path than would cars or other smaller vehicles. An unusually long time in queue may be a precursor to vehicles suddenly cutting out of line, possibly at a risk to oncoming traffic.

\subsection{SENSOR MEASUREMENT OF AGGREGATE PARAMETERS}

Table 5.4 is an attempt to quantify the degree to which the overall aggregate traffic conditions can be determined with the TFWAS system technology. Aggregate traffic parameters are such factors as coverage, velocity, acceleration, density, and extent. "Coverage" is taken to mean how much of the total area being monitored the individual sensor can actually "see," that is, how well the sensor covers its specific location. An inductive loop, for example, is "limited" because its coverage is very localized and may require multiple sensors. A microwave sensor might represent a "moderate" coverage since it can discern traffic over a more expansive range. "Extensive" coverage would be said of those sensors that can discern traffic conditions from a half mile to several miles, although the practical limitation may be on the order of 3 to 5 miles. All of the imaging systems, and definitely the probe vehicles, would have an extensive capability.

Velocity and acceleration would probably be analyzed together to ascertain changes in traffic flow, such as slowdowns, rush-hour stop and go, or sudden changes to normal flow. Acceleration would typically be derived from the rate of change of velocity. The capability of any given sensor to measure velocity, however, can be classified into three categories. The sensor capability is limited if it can only provide a qualitative estimate of the aggregate traffic velocity with little or no direct data output for later analysis. Passive infrared where the heat from the passage of multiple cars obscures individual discrimination is an example. The 
capability is moderate if the sensor can provide a limited quantification of the aggregate traffic velocity and is capable of some direct quantified data output for later analysis. Again, inductive loops meet this criterion for a given traffic lane. A sensor that can quantify velocity for virtually every vehicle passing would rate high. Acoustic sensors with Doppler algorithms would be an example.

Vehicle density is the quantification of the number of vehicles located adjacent to one another (i.e., the "clumps" of vehicles). The discriminators for density are similar to those for velocity. The capability of a sensor to provide only a qualitative estimate of the aggregate traffic density with no direct quantified data output for later analysis is said to be limited. Probe vehicles may only have the benefit of a verbal report from the driver. A sensor with moderate capability would provide a limited quantification of aggregate vehicle density both through real-time observation and through information stored for future analysis. Passive infrared recording concentrations of heat sources would be an example. High discrimination would derive from a sensor capable of quantifying and summing each vehicle passing. Many of the sensors are capable of discriminating density very well but are constrained to the lane within which they are installed. Those are identified in Table 5.4.

Extent is a very subjective parameter where the intent is to convey to the reader some insight as to how well the specific technology can apply to and perform the TFWAS function and how much aggregate traffic status information can be related to and derived by the observer. If only a limited amount of specific information is received, rendering the data either marginal or inadequate to determine overall traffic status, then the extent of the particular sensor is said to be limited. Sensors limited to single-lane detection would generally fall into this category. Sensors capable of integrating some events and presenting a broader scope of information enabling an observer to determine some trends would encompass a moderate extent. An extensive extent would be derived from a technology that would contribute significantly to the TFWAS system function and present sufficient information to allow an observer to adequately determine the overall traffic status. Video imaging and probe vehicles could both provide that function.

In summary, there is probably no single sensor technology capable of satisfying all sensing requirements of wide-area surveillance. Multiple sensors of different technologies will most likely be required and implemented. Furthermore, the growing needs of traffic control will undoubtedly drive the desired direction of future sensor development. The technology maturity, the sensor sensitivity, and the implementation and operational simplicity will all contribute to maximizing the probability of success. The inherent costs associated with fielding special instrumentation for wide-area surveillance is an especially critical parameter, and the cost-effectiveness of any sensor deployment must be considered. A discrete suite of sensors would in all probability be selected and specialized to the requirements of a specific application. Successful implementation will surely result from some form of information fusion where each sensor provides data output that is linked to a common information display. More sophisticated sensor technology emplacements may even capitalize on sensor and/or data fusion. Data fusion is the process by which the output information from two or more sensors is processed and merged into a common display. Sensor fusion, on the other hand, is where multiple sensing functions are accomplished by the same instrument, and the resulting signals are processed by common electronics; the melding of the ensuing information occurs within the instrument itself prior to data processing. An example of this would be where the visible and infrared spectra are imaged through common optics, but the light is directed to separate detectors. The subsequent signal is then processed through the same electronics, resulting 
in an image where the output of both detectors has been electronically superimposed. Although the technology in this area is evolving, the need for considerable development still remains. 


\section{MEASURES OF PERFORMANCE}

Several measures of performance will be applied to assess the performance of a TFWAS system both on a global level and at a systems level. On a global level, the overall effectiveness of a TFWAS system will be assessed by how the operation of such a system changes measurable traffic flow parameters. At a systems level, the performance of the TFWAS system will be assessed relative to how well the TFWAS system measures and communicates data. The individual components of a TFWAS system will be assessed as well, with various parameters used to measure the performance of the sensor suites, the communications, and the machine cognition or data integration processing algorithms.

\subsection{GLOBAL LEVEL}

At the most global level, as implied throughout this report, overall traffic control is achieved by a system consisting of a sensor subsystem (TFWAS), an intelligent traffic control subsystem that makes decisions and actuates controls, and a TMC subsystem that supervises, oversees, and possibly overrides the control subsystem. At this level, the measures of performance are measures of traffic flow. The overall traffic control system effectiveness is assessed by how the operation of the system changes traffic flow parameters.

The NPP (1994) describes several measures of overall performance of a traffic flow control system. The objective of the traffic flow control system is to simultaneously minimize delay and maximize throughput while equalizing the traffic load. Thus, both from the viewpoint of the control function and assessment of performance of the system, measures of delay and throughput must be furnished by the surveillance system. A secondary objective is to reduce energy consumption and improve air quality.

In addition to simultaneously minimizing delay and maximizing throughput, the traffic flow control has a secondary objective of equalizing the load (NPP 1994). A measure of how well this is being done is a measure of "balance of flow" between different parts of the system. A similar measure is the degree of fragmentation of control over an extended area.

In an early implementation, one of the tasks of the TFWAS system will be to count the number of stops and delays in the traffic flow in its area. It is expected that one of the early payoffs of traffic flow control will be the reduction in stops and delays. As a hoped-for indication of success, transportation planners intend to use stop and delay data.

In its most visionary implementation, the traffic flow control system will optimize speed, people flow, vehicle flow, slug flow, fuel consumption, and pollution emission (NPP 1994). In some sense, the surveillance system is expected to provide data on all these quantities. The NPPl (1994) does not define speed, but most likely what will actually be optimized is slug velocity. In addition, link travel time, rather than a less specific concept of "flow," is likely to be an objective that lends itself to optimization. There is an intermediate step of deriving flow or occupancy from point detector readings. Flow is the easier to obtain, but occupancy is the better predictor of link travel time. The task of transforming point detector readings into occupancy data and then transforming the occupancy into link travel time appears to be the most promising approach but needs more research.

From the perspective of transportation planning (a function different from and complementary to traffic control), a crucial global measure of performance is vehicle miles traveled (VMT) (as defined in Chap. 5) (NPP 1994). The improvement (or degradation) of 
a system can be assessed by observing changes in VMT per unit time. IVHS planners consider this especially important since they expect IVHS to double VMT rates within 30 years, with virtually no physical increase in road capacity.

Other overall measures that affect transportation planning are energy use and air quality. It is an objective of traffic control systems to reduce energy use and improve air quality. The monitoring of both, while not directly used in the control function, can provide information on the overall effectiveness of the system. The TFWAS system will be able to make some environmental measurements but not others. It is unlikely that the TFWAS system can monitor fuel consumption. At best, it might deduce some indication of fuel consumption from its observations of pollutant levels. What it can observe, and what might have impact on control actions, are pollutant levels. In particular, the system might measure concentrations of $\mathrm{CO}, \mathrm{NO}_{x}$ lead, and hydrocarbon particulates.

\subsection{SYSTEM LEVEL}

At the system level, there are measures of performance of the wide-area surveillance system itself. As discussed in the section on the global level, the TFWAS system is expected to provide a conceptual view of traffic flow and specific data on delay (number and duration), throughput, link travel time, occupancy (of the road by vehicles, not of vehicles by people), vehicle density, slug velocity and acceleration, and ambient levels of selected pollutants. The performance of a specific surveillance system is a measure of how well the system provides these quantities.

The measures of performance of a sensor system include reliability, repeatability, reproducibility, and accuracy. Reliability is a measure of the availability of the system and might include such measures as mean time between failure, percent downtime or percent uptime. Repeatability is a measure of the ability of the system to produce the same output for repeated instances of a given input; for control functions, repeatability is often more important (and cheaper to obtain) than absolute accuracy. Reproducibility is a measure of the ability of a unit of a system to produce the same output for a given input. Accuracy is the measure of the precision of a measurement or how far it deviates from a recognized standard; the cost of a sensor system can increase exponentially as a function of accuracy. Thus, it is often not cost-effective for a sensor to provide more accuracy than the control function really needs.

As indicated in Chap. 4, in addition to acting as the input transducer for a traffic flow control system, a TFWAS system is also expected to function as an incident detector. That is, in addition to the usual traffic control functions, it is intended to provide quick notification to emergency response and other services. Performance of TFWAS system as an incident detector can be measured by comparing its results against actual incidents known to occur.

Traffic controllers and emergency responders will always become aware of incidents, eventually. What the TFWAS system provides is fast notification. In assessing how well a TFWAS system does the job, three questions must be answered. How many false alarms (incident reported when none occurred) does the TFWAS system report? How many incidents does it fail to report? How much faster is the TFWAS system at reporting incidents than other forms of reporting, such as phone calls from concerned citizens? 


\subsection{SUBSYSTEM LEVEL}

As was emphasized in the Concept Definition document (Allgood, Ferrell, and Kercel 1994), the TFWAS system is not simply a collection of sensors spread over a wide area of highway. Rather, it is a system that produces a conceptualized measure of overall traffic flow, based on the input from many sensors.

The major unsolved problems involve the integrating principle. That is, how is the global conceptual view of traffic flow deduced from a torrent of sensor data? Integration of sensor data to obtain usable output will require at least six other technologies.

\subsubsection{Sensors}

Since the TFWAS system is a sensor in the global sense, so the individual sensor elements are in the local sense. Although different sensors detect different effects and yield different outputs, there are some general indications of performance that all sensors must meet. Thus the measures of performance of an individual sensor are akin to those for the overall TFWAS system. As already mentioned, these include reliability, repeatability, reproducibility, and accuracy.

\subsubsection{Communications}

Regardless of the communications technology used, the objective is to get data from one part of the TFWAS system to another or from the TFWAS system to the control system. The performance questions are as follows. To what extent is the communications link available? Is the receiver hearing what the sender is saying? Does the link have sufficient capacity?

Error rate, as in the number of erroneous bits per million bits decoded at the receiving end, is probably the best indication of the overall utility of a link. Other measures, such as signal-to-noise ratio, are only useful in that improving their values usually reduces the error rate. Throughput must be considered along with error rate. Sophisticated digital communications systems often use error correcting codes and "handshaking" to retransmit garbled data. The practical result is that over a noisy link, the data that get through are extremely accurate, but the throughput in the sense of error-free bits received per day might be significantly slowed down by retransmissions. Availability is probably best measured as the percent of time the link is available. This measure provides guidance to maintenance and determines the need to provide redundant links. Availability is an important factor in determining if a given technology is really best suited for a particular link.

\subsubsection{Machine Cognition}

A full-featured general-purpose TFWAS system would use broadly based cognitive algorithms to discern various attributes that characterize the traffic flow pattern. As the NPP (1994) recognizes, no such general algorithms exist but need to be developed. Currently in use are specific algorithms to perform limit functions for a set of precisely defined circumstances.

The primary measure of performance of a cognition algorithm is whether it correctly deduces the pattern of traffic flow from the data presented to it. The limiting issue on sophisticated algorithms is the ability to perform fast enough. Processing speed is a key measure of performance of a machine cognition algorithm. How long does it take the 
algorithm to turn a stream of sensor percepts into a concept of overall traffic flow? Scalability, or order of complexity, is another key measure of algorithm performance. If it works for a small system, will it also work for a big system? Finally, there is the issue of the believability of the concepts generated by the system. This may not be directly measurable. How good the cognition is may be only implicitly recognized by observing the higher level performance of the TFWAS system as different cognition algorithms are tried on the same data.

\subsubsection{Systems Integration}

There are several measures of performance in a parallel-distributed system (Mariani 1984). Since contention among processors for shared resources (common data bus, etc.) is often the limiting factor, the percent of time the processors are idle, waiting for their turn to use shared resources, is a critical measure. Processor benchmarking is the normal way of assessing the individual processors in the system. Benchmarking measures the total time for the processor to execute some standardized test-case algorithm. Particularly when comparing different kinds of processors, benchmarking is a more reliable indicator of performance than MIPS (millions of instructions per second).

Since it is not known whether processor speed or contention for resources is the limiting effect, it is also necessary to measure the overall performance of the parallel-distributed processing system. Important measures are central processing unit utilization, task utilization, queue length, queue wait times, task run times, and overall run times. 


\section{CONCLUSIONS}

TFWAS should not be confused with its components; it is not merely a collection of sophisticated sensors. TFWAS is an integrated sensing system that acts as an input transducer to a traffic control system to provide the traffic flow pattern. Sensing, communications, cognition/data integration, control strategy, human factors, system design, and large-scale system integration are all necessary to implement a TFWAS system.

Although several new technologies are being developed for traffic sensing systems, most commercial products brought to market using these alternative sensors function to perform detection and measurement as an inductive loop or as multiple inductive loops. That is, these systems seek to emulate the use of inductive loops to provide traffic information. With the exception of a few video image processing systems that employ tracking strategies, true surveillance of entire areas is not being accomplished. This may be the result of supply and demand. Since control strategies have been adapted to the standard of the inductive loop, there is no real demand for a true wide-area surveillance function. Similarly, since there is not a clear understanding of what kind of real-time information can be made available to the control system, control strategies have not been developed which can take advantage of such information.

Wide-area surveillance is a concept that can take advantage of the strengths of different traffic sensing technologies, can readily adapt to newly developed technologies, and can grow with the development of new traffic control strategies. By developing algorithms that will take information from a variety of sensor types and develop descriptions of traffic flows over a wide area, a more comprehensive understanding of the traffic state can be provided to the control system to perform optimal real-time adaptive control actions over the entire wide area.

In the near term, wide-area surveillance technology development will be driven by the market, independently of FWHA research priorities. What can be expected is the expansion into traffic control of sensor technologies previously developed for other problems and the increased use of data fusion algorithms to maximize the efficiency of existing sensor suites. These will result in improved performance as compared to existing wide-area surveillance systems, a larger area surveilled, more accurate data, etc. The sensor technologies are either mature or are being aggressively developed. The problem for the future is what to do with the sensor data. The NPP (1994) recognizes that for a full-featured wide-area surveillance system, required as the transducer into an adaptive traffic control system in a fully implemented IVHS, the major unsolved problem is machine cognition. How does the surveillance system take a large set of data flows and from those conceptualize the pattern of traffic flow through the area under surveillance?

TFWAS has the potential to revolutionize traffic control strategies. TFWAS is designed to provide a global view of the overall state of traffic. Most present schemes are based on very limited knowledge of traffic conditions. This global traffic view provides more complete information for anticipatory control. TFWAS provides quick feedback on the overall effectiveness of a particular control action. TFWAS will reduce the burden on the control system by deducing flow pattern. 


\section{REFERENCES}

Adarsh, Pankaj 1992. "Can't Afford Satellites? Try Meteors," Dataquest, April.

Allgood, Glenn O., Regina K Ferrell, and Stephen W. Kercel 994 . Concept Definition of Traffic Flow Wide-Area Surveillance, ORNL/TM-12773, July.

Anderson, F. L. 1992. "Meteor Burst Technology for Data Collection Networks," Proceedings from the Special Session on Real-Time Data Acquisition, American Society of Civil Engineers, October, Portland, Ore.

Andrews, John, et al. 1994. "The IVHS Potential of Surface Detection Radar," Paper 94044, IVHS America 1994 Annual Meeting, April 17-20, Atlanta.

Astrom, K. J., and B. Wittenmark 1989. Adaptive Control Systems, Addison-Wesley, Redding, Mass.

Bertsekas, D. P. 1987. Dynamic Programming: Deterministic and Stochastic Models, Prentice-Hall, Englewood Cliffs, N.J.

Bierman, Howard 1991. "Personal Communications and Motor Vehicle and Highway Automation Spark New Microwave Applications," Microwave J., August.

Blosseville, J. M., et al. 1989. Titan-A Traffic Measurement System Using Image Processing," 2nd International Conference on Road Traffic Monitoring, "February, London.

Blosseville, J. M., et al. 1991. "Automatic Incident Detection Using Image Processing Techniques: A Specific System Used in INVAID," Proceedings of Second International Conference on Applications of Advanced Technologies in Transportation Engineering, August, London.

Brookner, Eli, ed. 1977. Radar Technology, Artech House, Inc., Norwood, Mass.

Carbonell, Jamie 1992. Machine Leaming, MTT Press, Cambridge, Mass., ISBN 0-262-53088-0.

Cassandras, Christos G. 1993. Discrete Event Systems: Modeling and Performance Analysis, Richard D. Irwin, Inc., and Aksen Associates, Inc., Burr Ridge, Ill.

Cerocke, Charles 1992. "Meteor Burst Communications Project," Proceedings of the National Traffic Data Acquisition Conference, Sacramento, Calif., Oct. 25-29, 1992.

Chang, Edmond Chin-Ping, and Su-Ha Wang 1994. "Improved Freeway Incident Detection Using Fuzzy Set Theory," 73rd Annual Transportation Research Board Meeting, Paper 940603, Washington, D.C. 
Cima, David 1992. "Passed Infrared Vehicle Sensors," Paper CJ025, Transportation Researach Board Annual Meeting, January 14.

Cohen, S., and Z. Ketselidou 1992. "DAISI: A Calibration Tool for Automatic Incident Detection Algorithms, Application on the Paris Ringway," IEE Sixth International Conference on Road Traffic Monitoring and Control, April 28-30, London.

Copeland, Jack 1993. Artificial Intelligence, Blackwell, Oxford, UK, ISBN 0-631-18385-X.

Dailey, D. J. 1994. "An Optimal Recursive Estimator for Detecting Traffic Anomalies Using Real Time Inductance Loop Data," 73rd Annual Transportation Research Board Meeting. Paper 940424, Washington, D.C.

Dalgleish, Michael, and Neil Hoose 1994. "IMPACTS Incident Detection System in Glasglow, Scotland," Proceedings of the IVHS America 1994 Annual Meeting, April 17-20, Atlanta.

Dickinson, K W., and C. L. Wan 1989. "Road Traffic Monitoring Using the TRIP II System," 2nd International Conference on Road Traffic Monitoring, February, London.

Dickinson, K. W., and C. L. Wan 1990. "An Evaluation of Microwave Vehicle Detection at Traffic Signal Controlled Intersections," IEE Third International Conference on Road Traffic Control, May 1-3, London, UK.

DOT (U.S. Department of Transportation) 1994. Development of IVHS Traffic Parameter Specifications, May.

Dress, W. B., and S. W. Kercel 1994. "Wavelet-Based Acoustic Recognition of Aircraft" in Wavelet Applications, Hoarld H. Szu, ed., Proc. SPIE 2242, pp. 778-791.

Enkelmann, W. 1989. "Interpretation of Traffic Scenes by Evaluation of Optical Flow Fields from Image Sequences," Controls, Computers, Communications in Transportation, selected papers from the IFAC/IFIP/IFORS Symposium, September 19-21, Paris.

Erdman, William 1993. "Wireless Communications: A Decade of Progress," IEEE Commun. Mag. 31(12), 48-51.

Flanagan, Patrick 1994. "Wireless Data: Closing the Gap Between Promise and Reality," Telecommunications 28(3) 25-30.

Fukuhara, Hiroshige, and Kunihiko Kurami 1994. "Essential Issues Involved in Radar-Based Collision Warning/Avoidance Systems," Paper 94056, IVHS America 1994 Annual Meeting, April 17-20, Atlanta.

Garner, Joseph E., Clyde E. Lee, and Liren Huang 1990. Infrared Sensors for Counting, Classifying, and Weighing Vehicles, CTR 3-10-88/0-1162-1F, PB92-226091, University of Texas, Austin, December. 
Glasserman, P. 1991. Gradient Estimation via Perturbation Analysis, Kluwer, Norwell, Mass.

Gordon, Robert L, et al. 1993. Communications Handbook for Traffic Control Systems, FHWA-SA-93-052, April.

Grenzeback, L. R., J. R. Stowers, and A. B. Boghani 1988. Feasibility of a National HeavyVehicle Monitoring System. National Cooperative Highway Research Program Report 303, December.

Hallenbeck, Mark, Tim Boyle, and Jennene Ring 1992. Use of Automatic Vehicle Identification Techniques for Measuring Traffic Performance and Performing Incident Detection, Final Report, PB93-142123, October.

Haykin, S. S. 1994. Neural Networks: A Comprehensive Foundation, Macmillan, New York.

Hecht-Nielsen, R. 1989. The Technology of Non-Algorithmic Information Processing, Addison-Wesley, Redding, Mass.

Ho, Y. C., and X. Cao 1991. Perturbation Analysis of Discrete Event Dynamic Systems, Kluwer, Norwell, Mass.

Hockaday, Steven, et al. 1994. "Spread Spectrum Vehicle Communications Network," The Proceedings of the 1994 Annual Meeting of IVHS America, April 17-20, Atlanta.

Hogberg, Henrik 1993. "Wide-Area Wireless Data: Currently Available Systems and Applications," Telecommunications 27(12) 33-36.

Hoose, N. 1989. "Queue Detection Using Computer Image Processing," 2nd International Conference on Road Traffic Monitoring, February, London.

Hughes 1993. Select and Obtain Vehicle Detectors, Task D Report for Detection Technology for IVHS, Contract Number DTFH61-91-C-00076, Hughes Aircraft Corporation and JHK \& Associates, December.

Hughes 1994a. Vehicle Detector Field Test Specifications and Field Test Plan, Task Report F for Detection Technology for MHS Contract Number DTFH61-91-C-00076, Hughes Aircraft Corporation and JHK \& Associates, January.

Hughes 1994b. Development of IVHS Traffic Parameter Specifications, Task A Draft Report for Detection Technology for MHS, Contract Number DTFH61-91-C-00076, Rev. 5, Hughes Aircraft Corporation and JHF \& Associates, February.

IVHS 1994. "UMTRI-ERIM to Demo Laser-Based System to Collect Driver Data," Inside IVHS, pp. 8-9 (June 6).

Kawashima, H. 1989. "Integrated System of Navigation and Communication in Japan," Control, Computers, Communications in Transportation, Pergamon Press, Oxford. 
Keiser, Gerd 1983. Optical Fiber Communications, Mc-Graw Hill, New York.

Killen, Harold 1986. Telecommunications and Data Communication System Design with Troubleshooting, Prentice-Hall, Englewood Cliffs, N.J.

Klein, Lawrence A. 1993. Sensor and Data Fusion Concepts and Applications, SPIE Optical Engineering Press, Bellingham, Wash., Tutorial Texts in Optical Engineering, Vol. TT14.

Komanecky, Mark, and Thomas Minior 1992. "Communications Infrastructures for Advanced Traffic Management Systems," The Proceedings of the 1992 Annual Meeting of IVHS America, Vol. 2, Newport Beach, Calif., May 17-20.

Kosko, B. 1992. Neural Networks and Fuzzy Systems: A Dynamic Systems Approach to Machine Intelligence, Prentice-Hall, Englewood Cliffs, N.J.

Kumar, P. R., and P. Varaiya 1986. Stochastic Systems: Estimation, Identification, and Adaptive Controh Prentice-Hall, Englewood Cliffs, N.J.

Lodge, John 1991. "Mobile Satellite Communications Systems: Toward Global Personal Communications," IEEE Commun. Mag. 30(11), 24-30.

Mariani, M. P. 1984. Distributed Data Processing-Technology and Critical Issues, TRW Series of Software Technology, Vol. 4, El Sevier, Amsterdam, ISBN-0-444-86796-1.

Mead, William C., et al. 1994. "Application of Adaptive and Neural Network Computational Techniques to Traffic Volume and Classification Monitoring," 73rd Annual Transportation Research Board Meeting. Paper 940735, Washington D.C.

Michalopoulos, Panos G. 1991. "Incident Detection Through Video Image Processing," Proceedings of the Second International Conference on Applications of Advanced Technologies in Transportation Engineering, August, London.

Michalopoulos, Panos G., et al. 1994. "Field Deployment of Machine Vision in the Oakland County ATMS/ATIS Project," Paper 94016, IVHS America 1994 Annual Meeting, April 17-20, Atlanta.

Nagel, Kai, and Michael Schreckenberg 1994. "A Cellular Automaton Model for Freeway Traffic," J. Phys. I 2221-29, December.

Nathanson, Fred E. 1969. Radar Design Principles Signal Processing and the Environment, McGraw-Hill, New York.

Newton, W. H., B. A. Frith, and I. A. Barbour 1992. "Use of Weigh-In-Motion Systems to Aid the Selection of Goods Vehicles for Enforcement Weighing," IEE Sixth International Conference on Road Traffic Monitoring and Control, April 28-30, London. 
NPP (National Program Plan for Intelligent Vehicle-Highway Systems) May 1994. Draft, IVHS America, Washington, D.C.

Olson, Robert A., et al. 1994. “Active-Infrared Overhead Vehicle Sensor," IEEE Trans. Veh. Technol, February.

Plocher, T. 1993. Presentation to IVHS America Committee on Advanced Vehicle Control Systems, Orlando, Fla., February 8.

Rameswami, Rajiv 1993. "Multiwavelength Lightwave Networks for Computer Communication," IEEE Commun. Mag. 31(2), 78-88.

Rasmussen, J. 1986. Information Processing and Human-Machine Interaction: An Approach to Cognitive Engineering, North Holland Series in System Science and Engineering, Vol. 12, El Sevier, Amsterdam, ISBN-0-444-00987-6.

Reinhart, Edward 1992. "Mobile Communications," IEEE Spectrum 29(2) 27-29.

Robinson, Enders A 1967. Statistical Communication and Detection with Special Reference to Digital Data Processing of Radar and Seismic Signals, Charles Griffin and Company, London.

Roe, H., and G. S. Hobson 1992. "Classification of Road Vehicles from Microwave Profiles," IEE Sixth International Conference on Road Traffic Monitoring and Control, April 28-30, London.

Rowe, S. E., and Verej Janoyan 1992. "Communication Systems Design for the Los Angeles Smart Corridor Project," The Proceedings of the 1992 Annual Meeting of IVHS America, Vol. 2.

Rubinstein, R. V. 1986. Monte Carlo Optimization, Simulation, and Sensitivity Analysis of Queueing Networks, Wiley, New York.

Sandbank, C. P. 1980. Optical Fibre Communication Systems, Wiley, New York.

Schadschneider, A., and M. Schreckenberg 1993. "Cellular Automaton Models and Traffic Flow," J. Phys. A 26, L679-L683.

Schlutsmeyer, Alan P. 1982. Wide Area Detection System (WADS), Jet Propulsion Laboratory, PB84-150473, September.

Schultz, William 1993. "Traffic and Vehicle Control Using Microwave Sensors," Sensors 10(10), October.

Shanmugan, K. Sam, and Arthur M. Breipohl 1988. Random Signals: Detection, Estimation and Data Analysis, Wiley, New York. 
Sisiopiku, Virginia, and Nagui M. Rouphail 1994. "Towards the Use of Detector Output for Arterial Link Travel Time Estimation: A Literature Review," 73rd Annual Transportation Research Board Meeting, Paper 940160, Washington, D.C.

Skolnik, M. I., Ed. 1990. Radar Handbook, 2d Ed., McGraw-Hill, New York.

Stevens, A., J. R. Spindlow, and G. R. Jones 1990. "Fibre-Optic Axle Sensors for Vehicle Data Collection," IEE Third Intermational Conference on Road Traffic Control, May 1-3, London.

Sullivan, T., G. H. Lloyd, and J. Bonnett 1992. "Weigh-In-Motion and the Automation of the Core Census," IEE Sixth International Conference on Road Traffic Monitoring and Control, April 28-30, London.

Sumi, H., et al. 1989. "Method of Measuring Travel Time by Using Ultrasonic Vehicle Profile Classifiers," 2nd International Conference on Road Traffic Monitoring, February, London.

TDH (Traffic Detector Handbook) 1990. 2d Ed., JHK and Associates, PB91-164228, July.

Weissman, David, et al. 1993. "Interoperable Wireless Data," IEEE Commun. Mag. 31(2) 68-77. 


\title{
Appendix A \\ CAPABILITIES OF NATIONAL LABORATORIES RELEVANT TO WIDE-AREA SURVEIILANCE
}

\begin{abstract}
Several Department of Energy (DOE) national laboratories and two other laboratories with highly visible transportation research work participated in a workshop on Wide-Area Surveillance (WAS) sponsored by ORNL in November 1993. The following participants were invited to share their opinions of what WAS means and how it should be applied: Argonne National Laboratory, Lawrence Berkeley Laboratory, Los Alamos National Laboratory, Idaho National Engineering Laboratory, Massachusetts Institute of Technology's Lincoln Laboratory, Pacific Northwest Laboratory, Jet Propulsion Laboratory, Lawrence Livermore National Laboratory, Sandia National Laboratory, and ORNL. The above laboratories which do not have sections included below did not respond to requests for information about their laboratory's special capabilities which could be applied to WAS or IVHS work. This does not preclude their actual participation in the field-only that no response was received. This appendix provides an index of special capabilities and technologies provided by the national laboratories.
\end{abstract}

\section{ARGONNE NATIONAL LABORATORY}

Argonne National Laboratory has a major initiative in the area of advanced transportation. Six elements of the initiative involve technical activities ranging from computer modeling to engineering research and development (R\&D).

- National Computing Consortium for Advanced Automotive Engineering: The first element of Argonne's Advanced Transportation initiative is intended to aid the design and manufacture of vehicles through the use of advanced computational techniques, including application of massively parallel computers. This work will be organized as the new National Computing Consortium for Advanced Automotive Engineering. The Consortium is a collaborative effort involving Argonne, Los Alamos, Lawrence Livermore, Oak Ridge, and Sandia national laboratories and the Big Three automakers-Ford, General Motors, and Chrysler. The Consortium will initially focus on computational methods for analysis. Application of computers and computational systems analysis should enhance the agility of U.S. manufacturing and reduce the amount of time needed to design new products and bring them to market, improve product reliability, reduce production costs, and raise overall product quality. In pursuit of these objectives, the Consortium will develop analytical capabilities in fundamental engineering and scientific disciplines and will integrate complex computer models into sophisticated systems analysis tools. Communications systems that will allow interactive design and analysis over widely distributed areas will be established through use of current state, regional, and federal networks.

- Advanced Battery Consortium: In response to the increased public awareness of environmental impacts from vehicles, the U.S. Advanced Battery Consortium was formed to address the design of alternative power sources that are more energy efficient and less 
polluting than the presently dominant internal combustion engine. In support of the Consortium, Argonne will perform research and development for five battery technologies for electric and hybrid vehicles.

- Hybrid Vehicles: DOE has an initiative to develop hybrid propulsion systems to overcome the major shortcomings of battery-powered electric vehicles. Argonne is currently the lead DOE laboratory for R\&D on fuel cells for transportation.

- Vehicle Recycling: Argonne is currently negotiating a cooperative R\&D agreement to examine issues associated with recycling obsolete vehicles. Research will identify and quantify waste streams, determine impediments to recycling the waste streams, develop technologies to overcome those impediments, examine options for minimizing waste streams, and determine which options are most cost-effective, energy efficient, environmentally acceptable, and institutionally acceptable.

- Maglev Technology Development and Evaluation: Argonne is DOE's lead technical laboratory in a cooperative government-industry program to develop the next generation of U.S. maglev technology. Argonne has developed and validated computational codes and built small-scale experimental facilities to help the government evaluate and test alternative maglev designs. Initial experiments will test new components for levitation, guidance, and control of vehicle motion. The R\&D by Argonne will also address health effects associated with exposure to magnetic fields. It will also address energy storage, sensors for monitoring component performance and system parameters, computer codes for analyzing experimental results, and the development and evaluation of new concepts.

- Steel-Wheel Railroads: Argonne and the Association of American Railroads are developing an agenda for cooperative research in the areas of fuel cells, advanced materials, structural analysis, sensors, fracture, wear, environmental remediation, and risk assessment.

In addition to activities associated with the Advanced Transportation initiative, Argonne has expertise in other areas applicable to WAS, such as communications, sensors, and information management.

- Transcom: Transcom is a DOE system that provides a central monitoring and communications center for DOE shipments of spent fuel, high-level waste, and other high-visibility shipping campaigns. Using satellite communications, computerized database management, user networks, and ground communications with shipments, DOE can continuously monitor the location and status of these shipments within the United States. Argonne National Laboratory managed and directed the project in the development phase.

- Sensor Systems: Argonne has developed a millimeter wave system that uses advanced signal processing techniques to produce real-time images. A similar system designed for traffic monitoring could be developed. Argonne has applied ultrasonic imaging techniques for nondestructive evaluation and defect detection. Argonne has developed air-coupled ultrasonic transducers and wavelet signal processing techniques that might be applied toward traffic surveillance. 
- Information Management: The Decision and Information Sciences Division specializes in the development and application of innovative approaches to resolving problems in data management, decision analysis, database management systems, and information retrieval. The Visual Intelligence and Electronic Warfare Simulation provides an environment in which an analyst can access a reference database and build, modify, and display all aspects of a detailed battlefield scenario and generate a simulated sensor environment. The Dynamic Environmental Effects model allows a framework for environmental modeling and visualization for analysis. Objects created within the environment can be embedded with knowledge about their own response to environmental stimuli.

\section{IDAHO NATIONAL ENGINEERING LABORATORY (INEL)}

In INEL's Advanced Transportation Systems initiative, they will continue activities in the following thrust areas:

- The Electric and Hybrid Vehicle Program conducts testing and development of electric vehicles, batteries, and other potential power sources such as ultracapacitors.

- The prototype of an energy-efficient high-speed rail system has been tested at INEL.

- Research is being conducted in the areas of high-speed, high-volume data transmission techniques for control of advanced transportation concepts, such as rail transit and intelligent vehicle and highway systems.

- A materials processing initiative is proceeding, with a new focus on lightweight materials.

R\&D for other initiatives and programs is conducted at INEL which may be adaptable to traffic applications.

- RF and Microwave Technology: A Laboratory-directed R\&D initiative to develop an improved collision warning and avoidance capability for the advanced vehicle control systems in automobiles is under way. This effort initially concentrated on improving the data processing element of the system but now encompasses improving the sensor hardware.

- Video Image Processing: INEL is developing software for rapid image identification for identifying a wide range of military targets. An inexpensive PC platform, with parallel processing boards to provide the high-speed computations, may make this system adaptable to traffic surveillance targets.

\section{JET PROPULSION LABORATORY (JPL)}

JPL has substantial experience in conducting and managing Department of Transportation (DOT)-sponsored $R \& D$, including project management and the development 
of specific transportation-related technologies. In addition, an extensive amount of R\&D relevant to transportation has been conducted for non-DOT sponsors:

- Management of the IVHS System Architecture Development: JPL manages and guides the efforts of four teams engaging in developing possible system architectures for IVHS. This architecture must support the surveillance, communications, data management, control software, and display technologies necessary to help solve congestion, safety, and productivity problems associated with the transportation of goods and people.

- Wide-Area Detection System/Scanning Technology. JPL initiated some of the first U.S. studies on wide-area detection in the early 1970 s and recognized its potential for improving traffic control strategies.

- Voice Switching and Control System Project: The Federal Aviation Administration (FAA) is sponsoring a project to provide a highly reliable state-of-the-art voice switching and control system which allows FAA controllers to communicate with aircraft and supporting ground facilities automatically. JPL's role is to develop, deliver, operate, and enhance a traffic simulator unit to drive and evaluate the system under test conditions, to specify voice system requirements, and to technically and programmatically monitor the prototype and production contracts.

- Development of Automated Mixed Traffic Vehicle: This project involved both project management and technology development. Technologies included vehicle design and development, collision avoidance/proximity sensors, vehicle controller, and test and evaluation.

- Real-Time Weather Processor Project: JPL has demonstrated strong system integration skills in the transportation arena in its work on the Real-Time Weather Processor Project. The FAA sponsored this system to process weather data by creating a mosaic display of up to 27 radars simultaneously, superimposed on aeronautical map backgrounds. Data are disseminated directly to air traffic controllers and meteorologists. JPL designed, developed, integrated, tested, and delivered the software and prototype hardware set for processing radar weather data.

Through its primary role of supporting the space science community, JPL has evolved a well-earned expertise in the development of innovative sensors and instruments, in the analysis and modeling of these sensor systems, and in the processing and visualization of the resulting data. Capability in sensor development and implementation covers the electromagnetic spectrum from $X$ rays to the microwave region. Responsibilities have traditionally centered around the conception, acquisition, engineering development, management, and support of space observational systems but have been applied to nonspace applications as well. Two camera systems developed by JPL for space applications may be adapted for traffic surveillance.

- JPL Wide Field/Planetary Camera II: This system was used to very successfully correct images from the Hubble Space Telescope. This is an example of the JPL expertise in conception, advanced technology, design, implementation, and data reduction related to instrument systems. 
- Pluto Integrated Camera System: This camera system is capable of simultaneously imaging in both the infrared and visible regimes. This is an excellent example of evolving technology in sensor and data fusion which might be relevant to surveillance and information processing for traffic monitoring and control.

JPL's system's engineering capability has been an important component for all these projects. JPL has developed special competency in the areas of architecture and system design for distributed data base systems, sensor data fusion and correlation, message-based information systems, and secure information systems.

\section{LAWRENCE BERKELEY LABORATORY}

Although Lawrence Berkeley Laboratory does not have a major initiative in transportation, research is being conducted in several applicable areas.

- X-Ray Lithography Sensors: Lawrence Berkeley is a leader in the development of sensors using X-ray lithography. Their goal is to manufacture nano-sized sensors. There is an active program in multiple sensor devices, including the hardware and software used to interpret and control these signals. Pixel matrix detectors and a variety of solid state detectors are also being developed.

- Energy-Demand Forecasting Models: The Energy and Environment Division maintains a range of energy-demand forecasting models that can be applied to the transportation sector. Data or methodologies for these models can be supplied by the Division's specialists in standards analysis and compilation of monitored data. Another focus of the Energy and Environment Division is pollution prevention and abatement.

\section{LOS ALAMOS NATIONAL LABORATORY}

Although not a major program, Los Alamos does have an initiative to assist the DOT.

- The FHWA has funded an important new start in the Transportation Analysis Simulation System. This is a modeling and simulation effort utilizing Los Alamos' vast computational capabilities. Los Alamos expects to participate in modeling and in demonstrations of sensors and signal processing for the IVHS.

- Los Alamos intends to address the Automated Highway System, part of the IVHS, with a completely new system of sensors and communication for cooperative collision avoidance cruise control.

- Los Alamos is also applying its engineering modeling capabilities in a project to study nondestructive evaluation techniques on bridges.

- Los Alamos has also demonstrated that the LIDAR satellite is a powerful tool for measuring air quality associated with vehicle emissions. Experimental campaigns have 
located sources of pollution, tracked the movement of aerosols, and located mixing-layer boundaries as a function of time and traffic conditions.

\section{MASSACHUSETTS INSTITUTE OF TECHNOLOGY LINCOLN LABORATORY}

Lincoln Laboratory's primary mission is the development of surveillance systems. The principal technology areas of focus for the facility are radar measurements, surveillance and control, optical systems, communications, air traffic control, solid state electronics, machine intelligence, and satellite systems. Approximately $10 \%$ of the research funding at the facility is from the DOT. Current R\&D at Lincoln Laboratory encompasses several areas of expertise that may be applicable to WAS:

- Project Control System Simulation: This project involves design refinements to the Central Artery/Third Harbor Tunnel project linking Boston to Logan airport. A microscopic simulation was written to simulate up to 30,000 vehicles responding to ATMS signals.

- IVHS Research: A study of the technology needs of the IVHS was completed. Included in the study were several recommendations for field demonstration projects.

- Airport Traffic Surveillance: The Massachusetts Institute of Technology Lincoln Laboratory has been conducting research under FAA sponsorship at Boston's Logan Airport. The laboratory has performed a significant amount of work in air traffic monitoring, control, and simulation. One of the most applicable tasks to WAS involves work with a surface detection radar at Logan airport. The goal of this work is to effectively track airplane and vehicle surface traffic with high reliability to ensure safe vehicle movement within the airport. Lincoln Laboratory modified a commonly used marine radar and performed specialized signal processing to reduce clutter and eliminate data from areas that are not of interest. This system has proven to be highly reliable. Many of the techniques used for clutter and multipath reduction might be adapted and modified to meet the purpose of a street traffic area surveillance detector (Andrews et al. 1994).

- Human Factors: A series of flight tests was flown to measure pilot air-to-air visual acquisition performance for pilots employing unalerted visual search. Results were analyzed to estimate the instantaneous rate of visual acquisition for a visual target of specified size and contrast. The results were used to calibrate a mathematical model of visual acquisition that can be used to predict pilot performance under a range of conditions.

- Sensor Fusion/System Integration: Integration of multiple sensor technology has been accomplished in several endeavors, including an aircraft-based multisensor system using laser radars, longwave passive infrared, and millimeter wave radar.

- Microwave Radar Systems: A great deal of the research at Lincoln Laboratories is centered on radar systems design, radar system enhancements, and the enhancement and processing of radar data or imagery. They have developed an ultra-wideband imaging 
radar for Wright Laboratory capable of 2- or 3-D imaging. They have developed a millimeter wave SAR imaging system to detect and classify stationary targets.

\section{OAK RIDGE NATIONAL LABORATORY}

The ORNL Institutional Plan sites Transportation Technologies and Systems as a major initiative. This initiative is contained in the broader Oak Ridge Transportation Center, which is being undertaken by Martin Marietta Energy Systems and includes programs at the Oak Ridge Y-12 Plant and Oak Ridge K-25 Site as well. ORNL intends to work in close collaboration with $\mathrm{Y}-12$ and $\mathrm{K}-25$ in the areas of hybrid vehicle technology, materials, emissions reduction, advanced manufacturing processes, and transport of hazardous materials. ORNL intends to expand capabilities in the following areas:

- Automotive Manufacturing: ORNL and Y-12 have capabilities in the areas of high-precision machining, hydroforming, design and fabrication, application of highperformance computing, and process certification.

- Hybrid/Clean Vehicle: ORNL envisions participation in the development of propulsion systems, permanent magnet motor-generators, power electronics, flywheels, and advanced magnetic materials for vehicles with hybrid electric propulsion systems and lightweight bodies.

- Intelligent Vehicle Highway Systems: ORNL has capabilities in advanced computing, communication, and control technologies. Continuing and prospective research includes crash avoidance systems, measurement of vehicle energy efficiency and emissions, vehicle guidance and control, vehicle and driver diagnostics, traffic control simulation, advanced traffic control centers, human factors in system design, fast computing and applicationspecific integrated circuits, dynamic route planning, and system evaluation methodologies. ORNL is also leading the effort to establish a regional coalition to conduct and evaluate operational tests of advanced vehicle highway technologies.

- Infrastructure Modernization: ORNL's expertise in structural engineering, materials science, and sensor design would be applied to problems of premature deterioration of pavements and bridges.

- Acoustic Signature Analysis: ORNL has pioneered the use of wavelet analysis to classify vehicles moving on the ground by their acoustic signatures.

- Human Factors: ORNL has done extensive work in human factors for control rooms, human response to simulators, and the response of drivers to impending crashes. ORNL is currently developing DASCAR, a human factors instrument package for the National Highway Traffic Safety Administration (NHTSA).

- Hazardous Materials: ORNL has experience in the areas of packaging and transporting hazardous materials. 
- Transportation Planning and Analysis: ORNL can provide the continuing analysis and assessment required to integrate the R\&D with policy actions and to redefine research priorities.

ORNL's Center for Transportation Analysis receives funds from the DOT and covers all transportation modes; however, it focuses on highway transportation. ORNL is assisting the FHWA and will assist the newly developed Bureau of Transportation Statistics in several research areas: the development of freight and passenger demand models, assessment of data quality and data consistency of highway statistics, development of data collection methods, advanced data management systems to improve data integrity and availability, analysis of nationwide surveys to address issues in current or future national transportation policies, development of methods to statistically link data sources to study intermodal traffic, and research on IVHSs.

To support a range of analytical efforts, ORNL has been the leader in the development of an extensive national highway network database that contains roadway lengths, traffic capacities, and other statistically and operationally useful planning characteristics for about 400,000 miles of highway. Workstation versions of this database are being developed for use in a variety of graphically enhanced highway research applications.

ORNL plans increasing involvement in research projects that study highway safety from the viewpoint of geometric design, highway systems operation, and hazardous materials transport. The application of expert system methodologies to traffic simulation is emerging as a research area. For the National Highway Network Evaluation and Design Project, ORNL will generate a number of candidate highway networks with goals such as traveller mobility, equity, intercity trade, and connectivity for national security reasons. ORNL is also working with the FHWA to develop real-time monitoring and routing models. WAS.

Several groups at the Laboratory have specific capabilities that may be applicable to

- The Center for Computational Sciences, a part of the Engineering Physics and Mathematics Division, has expertise in image processing, computer vision, pattern recognition, laser range image analysis, surface recognition, sensor fusion, machine learning, parallel computing, and distributed memory techniques.

- The Cognitive Systems and Human Factors Group, also of the Engineering Physics and Mathematics Division, conducts various work applicable to transportation. The group was established in 1978 primarily in support of human factors activities in support of the NRC. The group is multidisciplinary with expertise in psychology, engineering, and computer science. The group is best known for its work in man-machine interface design, simulation and modeling, and human factors for advanced instrumentation and control.

- The National Highway Safety Administration is sponsoring the design, development, and evaluation of a prototype Data Acquisition System for Crash Avoidance Research. The system installed on a vehicle will collect a number of performance measures pertaining to the human driver, the vehicle, and the environment in or on which the vehicle is operated. 
- The FHWA is sponsoring the development of a prototype for training human controllers working with advanced traffic management system technologies. The prototype will include a number of advanced workstations, computer-generated traffic displays, and a trainer's console. It will be capable of running various types of traffic control software such as SCOOTS and will be able to automatically collect a number of human and system performance measures associated with controllers addressing various types of traffic scenarios.

- Another project sponsored by the FHWA is the development of an in-vehicle signing system. The system will provide signing such as speed limits, warning of congestion ahead, or directions to the driver of the vehicle. The system will likely involve both tactile and voice I/O capabilities. Initial efforts will focus on the identification of functional and technical specifications, with specific emphasis on human factors issues such as cognitive overload and attention allocation.

- ORNL is also part of a consortium to design, develop, and operationally test an enroute advisory system for Atlanta capable of interacting with the IVHS infrastructure. The system will include on-board navigation systems and will receive real-time traffic advisories. ORNL's contribution to this effort involves the design of the system interfaces, development of a system experimental evaluation plan, and participation in the evaluation and subsequent analysis.

- Chaos theory has been applied by ORNL's Engineering Technology Division, as well as time series analysis with pattern recognition. The division performs work in various areas related to transportation: improving sensors for emissions control, use of computers for design and testing combustion technology, and ignition evaluation of diesel fuels.

- The Optical Spectroscopy Group is developing new approaches to chemical sensing. Their approach involves miniaturization through microfabrication techniques of conventional chemical measurement techniques. The group also is experimenting with fiber-optic probes for chemical sensing and diode laser-based techniques for process control.

- Robotics and Process Systems Division has expertise in laser based-systems. Some of their work includes range sensing, surface mapping, and position and orientation tracking for vehicles done by sweeping planes of laser light across the work area and using triangulation.

- The Health Sciences and Research Division is developing the Center for Risk Management. The center will be used to measure and assess the impact of radiological and chemical substances on human health. The center will focus on improving the understanding of pollutant interactions at the atomic and molecular levels, developing image technologies for a broad range of biological samples, creating hybrid instruments for chemical mapping, and developing advanced photonic devices for environmental and biomedical applications. 
- The Advanced Methods Group has expertise in advanced signal processing techniques. They are currently engaged in research in noncontact airbag activation sensing for the NHTSA.

\section{SANDIA NATIONAL LABORATORIES}

Sandia has a major initiative in Transportation Energy Technology and Infrastructure. Sandia has been the lead laboratory for transportation technologies associated with the safe, reliable, and monitorable movement of defense hardware and radioactive and hazardous materials. This role includes infrastructure issues in geographic information system tracking of trucks, full vehicle system design and production oversight, and reliability and safety integration. Sandia's energy-related transportation responsibilities have been primarily focused on engine combustion technology and advanced batteries.

Sandia's Advanced Transportation Energy Technology Program is focused on improving the energy efficiency of our transportation system. The following projects provide a foundation for expanding effort in developing solutions:

- Advanced Energy Storage: Sandia has considerable expertise in energy storage development, including new developments in capacitors and batteries. This expertise is being applied to support the Advanced Battery Consortium.

- Hybrid Vehicles: Sandia's battery technology base and its combustion expertise, located at DOE's Combustion Research Facility at Sandia, coupled with their systems engineering capabilities will be applied to the development of hybrid vehicles.

- Alternative Fuels: Sandia's Combustion Research Facility will support work in optimizing engine designs for emissions control and high efficiency, use of alternative fuels in fuel cells, and use of hydrogen as a fuel.

- Emissions Consortium: Sandia will contribute by developing sensors and controls to understand the distribution of exhaust gases.

Another current initiative is to direct Sandia's core competencies in the areas of systems integration, sensors, data fusion, communications, and information and control to the IVHS. $R \& D$ in several areas will be conducted to foster rapid commercialization.

- High-Speed Computation and Systems Analysis: Sandia's experience in modeling and analysis will be used to perform large-scale computer simulations and systems analyses at all levels of system design. A current project is to examine the technical and economic feasibility of the proposed Santa Teresa Intermodal Facility.

- Intelligent Vehicle Highway System: Sandia has developed many types of robust, unattended ground sensors for monitoring commercial vehicle safety, "weighing" passing trucks, and automatically assessing tolls. They have also developed many versions of teleoperated and autonomous vehicles. 
- Transportation of Radioactive and Hazardous Materials: Sandia supports DOE in transportation-related efforts in several ways, including the development of transportation packagings. Sandia also supports the Transportation Management Program with efforts in the areas of policy analysis, packaging improvements, regulatory and standards support, testing and development of materials for design, and testing of packages.

- Alliance for Transportation Research: Sandia has entered into an alliance with Los Alamos, the University of New Mexico, New Mexico State University, and the New Mexico State Highway and Transportation Department for transportation research.

- Transportation System Risk Assessment: Sandia can assist DOE in its responsibility to regulate the nation's energy supply and can also help DOT ensure environmental, safety, and health compliance and promote safe transportation. Sandia is well positioned to conduct risk assessment studies and testing from a systems-level point of view, addressing both safety and security. As the originator of probabilistic risk analysis methodologies fornuclear power systems and nuclear weapons, they can apply the same or similar techniques to address economic issues and risk-related scenarios.

Sandia's extensive expertise in sensors and sensor integration includes technologies that may be further developed and adapted to traffic applications.

- Laser Radar Sensor: Sandia is developing a scanless laser radar imaging device capable of simultaneously producing a range image and a reflectance image. Traditional laser imaging techniques involve scanning a concentrated beam to obtain an image. Laser diodes are used in the source, which requires a large beam divergence equal to the field of view for the application. The result is a cheaper, faster, more rugged, and safer system than traditional laser range finders. Although this technology was developed for DOE surveillance applications, it has good potential for further development for traffic applications.

- Image Transmission System for Traffic Monitoring: Sandia has developed a low-cost image transmission system that transmits the first image normally and transmits subsequent images based on differences in the previous image. Sandia is collaborating with New Mexico state police to try using the system for traffic monitoring for planning purposes and to review accident causes.

- Sensor Integration: Sandia has demonstrated the capability for multiple sensor development and integration with the Advanced Exterior Sensor. Three sensing technologies, infrared, visible, and millimeter wave radar, are used to detect motion in a continuous $360^{\circ}$ field of regard. The lightweight, portable system was designed for robust operation in virtually any terrain or environmental condition. This sensor system was developed primarily for surveillance and robotics applications. 
REFERENCE FOR APPENDIX A

Andrews, John, et al. 1994. "The IVHS Potential of Surface Detection Radar," Paper 94044, IVHS America 1994 Annual Meeting, April 17-20, Atlanta. 
Appendix B

\section{A LOOK TO THE FUTURE}

Without FHWA support, WAS technology will develop in the near term as driven by the market. Sensor technologies previously developed for other problems will probably be expanded for use in traffic detection and control. An increased use of data fusion algorithms will maximize the efficiency of existing sensor systems. These developments will result in improved performance as compared to existing WAS systems, a larger area monitored, more accurate data, etc.

In assessing its research priorities in TFWAS, FHWA must consider where to invest for maximum return in the long term. Many sensor technologies are either mature or are being aggressively developed. One problem for the future is to determine ways to optimally use the sensor data. The NPP (1994) recognizes that for a full-featured WAS system, required as the transducer into an adaptive traffic control system in a fully implemented IVHS, the major unsolved problem is machine cognition. How does the surveillance system take a large set of data flows and, from those, conceptualize the pattern of traffic flow through the area under surveillance?

There is a particular kind of cognition that the National Program Plan says the full-featured TFWAS must exhibit, namely anticipation. There are some critical, but subtle, differences between an anticipatory system, a reactive system, and a prediction. It is worthwhile to consider the difference between a reactive system and an anticipatory one since much of the innovation and impact in our program plan hinges on the concept of anticipatory behavior. A reactive system bases its control actions on its presently sensed data; it can only respond to a problem once the problem occurs; it is somewhat limited in how it subsequently deals with the problem. An anticipatory system bases its control actions on past and present data, as well as predictive models of what will happen. This type system can take a proactive approach and try to prevent a problem from occurring in the first place. An illustration of the difference between the two is given in Appendix C. Prediction is a well-known technique used in signal processing and modern telephony. It is also a property of biological organisms.

Although anticipatory mechanisms are relatively rare in the world of man-made artifacts, they abound at all levels in the world of biological organisms. Indeed, much of what we consider to be typically and uniquely human behavior is anticipatory in nature. Anticipation appeared early in evolution as shown by the remarkable behavior of the horse-shoe crab's vision system (brain plus photoreceptors) that anticipates day-night changes to start a long process of turning up its "image intensifier" before dusk and down before dawn. If this system were reactive, the organism either could not see at night (by starlight or moonlight) or would be permanently blinded at dawn since a reactive system could not respond rapidly enough over the very large range of sensitivity.

One might say that this is an example of prediction. That would be correct, but it is prediction that is based on a good model of the diurnal cycles for the crab. It is genetic. Prediction without a model, or without subsequent action, is not anticipatory behavior. Prediction is a technique of anticipation, not the essence.

Based on the work of theoretical biologist, Robert Rosen, an anticipatory system can be defined to be one "in which the present change of state depends on future circumstances, rather than merely on the present or past" (Rosen 1985). Rosen goes on to observe that "Biology is replete with situations in which organisms can generate and maintain internal 
predictive models of themselves and their environments, and utilize the predictions of these models about the future for purpose of control in the present. Many of the unique properties of organisms can really be understood only if these internal models are taken into account. Thus, the concept of a system with an internal predictive model seemed to offer a way to study anticipatory systems in a scientifically and rigorous way" (Rosen 1985).

Our goal is not the analysis or study of biological organisms as anticipatory systems, but rather the inverse process, that of synthesis. The idea is to apply the understanding of organisms at the level of anticipatory systems. Some very exciting work has already been done based on anticipatory systems concepts. In particular, work accomplished at the University of Illinois (Tsoukalas 1989) has applied anticipatory control methods to the problem of nuclear power plant control.

It is clear from the example that an anticipatory system need not be overly complicated. What is important is the internal relationships of the subsystems and how they are implemented. The overall problem must be addressed in a coherent and consistent framework, not as a collection of techniques and sensors. What is needed is an integrated system that can anticipate changes in traffic flow patterns based on present knowledge of context and traffic flow data. A cross between a machine and a biological organism has the requisite properties. Such a system operates like an organism but is implemented in silicon and based on accepted engineering practice applied to reliable, robust, and inexpensive components. To accomplish what is needed, we need to incorporate the context of the highway environment system as closely as possible. This context becomes the model of the vehicle and environment that is an integral part of the anticipatory TFWAS.

It is fair to say that present progress in anticipation has been in the area of perception rather than cognition. Nobody knows how to write an anticipatory cognitive algorithm. The solution to the problem of applying machine cognition and anticipation to TFWAS is probably several decades in the future. So is the financial payoff. Consequently, this is unlikely to be undertaken by the present generation of private sector WAS developers. It is here that FHWA can invest in research that is critically needed for IVHS but offers neither certain short-term payoff to the private sector nor incentive for them to undertake the risk. There may, however, be valuable intermediate technical advances that can be implemented during the development cycle.

In a process such as traffic flow, where in a short period of time numerous directional and dynamic changes occur, the capability of predicting or anticipating probable behavior would be priceless. Its development would be a major technological and sociological breakthrough. The sociological benefit would be significant because of the reduced cost to society from the accidents that could be prevented, injuries avoided, and lives saved. On the order of 50,000 people a year are killed in automobile accidents, and over four times that many are maimed in some way. Millions of dollars are expended annually, both in private and public funds, in the form of support to people in and out of institutions with long-term disabilities, to the loss of job skills and jobs themselves, and to lives shattered as a result of traffic accidents. We pay heavily for this, and an anticipation technology could greatly reduce the burden. 


\section{REFERENCES FOR APPENDIX B}

NPP (National Program Plan for Intelligent Vehicle-Highway Systems) May 1994. Draft, IVHS America, Washington, D.C.

Rosen, Robert 1985. Anticipatory Systems, Pergamon, New York.

Tsoukalas, Lefteris 1989. Anticipatory Systems Using a Probabilistic-Possibilistic Formalism, Ph.D. thesis, University of Illinois, Urbana-Champaign. 
. 
Appendix C

\section{ILLUSTRATION OF DIFFERENCES BETWEEN REACTIVE AND ANTICIPATORY SYSTEMS}

Consider a camera with autoexposure built in. The mechanism measures the light value and computes a lens opening from the user-selected exposure time. The lens opening (f-stop) is then set to the computed value by the mechanism (or the f-stop is selected by the user and an exposure time is computed). A very simple model of the film-recording system is built into this mechanism; it is adjustable by the user, who sets a film-speed parameter. Such a camera contains a model of its own behavior as well as that of the film it is using. The camera anticipates that the light intensity measured just before the shutter is opened is that which will be needed to properly expose the film when the shutter is actually opened. This is an anticipatory system based on a simple mechanism.

How would a reactive camera operate? The film speed would likewise be a necessary parameter. There would be no presetting of the lens opening, so one would be restricted to setting the f-stop only. The act of taking a picture would be to open the shutter manually. The film or other device would report when enough light had entered the camera and close the shutter automatically. Note that it is possible to fool the anticipatory camera, but not the reactive camera. If we point the camera toward some dark corner just as we take a picture of a bright scene, the film will be underexposed, thus creating a situation that the simple mechanism could not anticipate.

The reactive camera has a much more serious drawback; its range of operation is severely restricted. Both the speed of the film and the length of exposure are limited by the reaction times inherent in the mechanisms themselves. The sensitivity and response of the light meter is not instantaneous; the mechanics of closing the shutter introduces delays. These time limitations restrict the range of exposure times over which the camera can operate. Estimates of such reaction times of modern components show that a reactive camera could only operate from " $B$ " to about $1 / 100 \mathrm{~s}$. The typical anticipatory camera (the only autoexposure type that is marketed) has a range from " $B$ " to at least $1 / 2000 \mathrm{~s}$, over 20 times larger. This added flexibility allows the user to employ a much wider range of film types, resulting in successful photographs in a wide range of environmental conditions. No one would seriously consider manufacturing and marketing a reactive camera even though it would be much harder to deceive. (A reactive submechanism could be designed to prevent the shutter closing should the light value decrease too much during an exposure. This would extend the utility of the anticipatory camera for those rare instances where it might be fooled.) 


\section{INTERNAL DISTRIBUTION}

1. R. A Abston

2-6. G. O. Allgood

7. C. L. Carnal

8. B. G. Eads

9-18. R. K. Ferrell

19. R. B. Honea

20. J. M. Jansen

21-22. S. W. Kercel

23. D. W. McDonald

24. G. N. Miller

25. G. T. Alley
26. J. D. White

27-28. A. K. Rathi

29. J. O. Stiegler

30. R. E. Uhrig

31. D. F. Craig

32-33. Central Research Library

34. Y-12 Technical Reference Section

35-36. Laboratory Records

37. Laboratory Records-Record Copy

38. ORNL Patent Section

39. I\&C Division Publications Office

\section{EXTERNAL DISTRIBUTION}

40-42. Alberto J. Santiago, Branch Chief, Federal Highway Administration, Office of Safety and Traffic Operations R\&D, HSR-11/Turner Fairbank Hwy. Res. Ctr., 6300 Georgetown Pike, McLean, VA 22101-2296

43. Assistant Manager for Energy Research and Development, DOE-ORO, P.O. Box 2001, Oak Ridge, TN 37831-8600

44. B. Chexal, Electric Power Research Institute, 3412 Hillview Avenue, Palo Alto, CA 94303

45. V. Radeka, Brookhaven National Laboratory, Instrumentation Division, 535-B, Upton, NY 11973.

46. M. M. Sevik, Carderock Division, Naval Surface Warfare Center, Code 1900 , Bethesda, MD 20084-5000

47. R. M. Taylor, Leeds and Northup, Sunneytown Pike, North Wales, PA 19454

48-53. P. I. Moynihan, Jet Propulsion Laboratory, MS 306-431, 4800 Oak Grove Drive, Pasadena, CA 91109-8099

54-55. Office of Scientific and Technical Information, U.S. Department of Energy, P.O. Box 62, Oak Ridge, TN 37831 